\title{
Models and data analysis tools for the Solar Orbiter mission
}

\author{
A. P. Rouillard ${ }^{1}$, R. F. Pinto ${ }^{1,21}$, A. Vourlidas ${ }^{2}$, A. De Groof ${ }^{3}$, W. T. Thompson ${ }^{4}$, A. Bemporad ${ }^{5}$, S. Dolei ${ }^{6}$,
} M. Indurain ${ }^{1}$, E. Buchlin ${ }^{19}$, C. Sasso ${ }^{15}$, D. Spadaro ${ }^{6}$, K. Dalmasse ${ }^{1}$, J. Hirzberger ${ }^{11}$, I. Zouganelis ${ }^{3}$, A. Strugarek ${ }^{21}$, A. S. Brun ${ }^{21}$, M. Alexandre ${ }^{1}$, D. Berghmans ${ }^{12}$, N. E. Raouafi ${ }^{2}$, T. Wiegelmann ${ }^{11}$, P. Pagano ${ }^{26}$, C. N. Arge ${ }^{14}$, T. Nieves-Chinchilla ${ }^{33,14}$, M. Lavarra ${ }^{1}$, N. Poirier ${ }^{1}$, T. Amari ${ }^{42}$, A. Aran ${ }^{29}$, V. Andretta ${ }^{15}$, E. Antonucci ${ }^{5}$, A. Anastasiadis ${ }^{32}$, F. Auchère ${ }^{19}$, L. Bellot Rubio ${ }^{16}$, B. Nicula ${ }^{12}$, X. Bonnin ${ }^{31}$, M. Bouchemit ${ }^{1}$, E. Budnik ${ }^{1}$, S. Caminade ${ }^{19}$, B. Cecconi ${ }^{31}$, J. Carlyle ${ }^{10}$, I. Cernuda ${ }^{30}$, J. M. Davila ${ }^{14}$, L. Etesi ${ }^{9}$, F. Espinosa Lara $^{30}$, A. Fedorov ${ }^{1}$, S. Fineschi ${ }^{5}$, A. Fludra ${ }^{7}$, V. Génot ${ }^{1}$, M. K. Georgoulis ${ }^{23,24}$, H. R. Gilbert ${ }^{14}$, A. Giunta ${ }^{7}$, R. Gomez-Herrero ${ }^{30}$, S. Guest ${ }^{7}$, M. Haberreiter ${ }^{18}$, D. Hassler ${ }^{13}$, C. J. Henney ${ }^{22}$, R. A. Howard ${ }^{8}$, T. S. Horbury ${ }^{41}$, M. Janvier ${ }^{19}$, S. I. Jones ${ }^{14,33}$, K. Kozarev ${ }^{27}$, E. Kraaikamp ${ }^{12}$, A. Kouloumvakos ${ }^{1}$, S. Krucker ${ }^{9}$, A. Lagg ${ }^{11}$, J. Linker ${ }^{40}$, B. Lavraud ${ }^{1,43}$, P. Louarn ${ }^{1}$, M. Maksimovic ${ }^{31}$, S. Maloney ${ }^{38,39}$, G. Mann ${ }^{36}$, A. Masson ${ }^{3}$, D. Müller ${ }^{10}$, H. Önel ${ }^{36}$, P. Osuna ${ }^{3}$, D. Orozco Suarez ${ }^{16}$, C. J. Owen ${ }^{17}$, A. Papaioannou ${ }^{32}$, D. Pérez-Suárez ${ }^{37}$, J. Rodriguez-Pacheco ${ }^{30}$, S. Parenti ${ }^{1}$, E. Pariat ${ }^{31}$, H. Peter ${ }^{11}$, S. Plunkett ${ }^{8}$, J. Pomoell ${ }^{35}$, J. M. Raines ${ }^{25}$, T. L. Riethmüller ${ }^{11}$, N. Rich ${ }^{8}$, L. Rodriguez ${ }^{12}$, M. Romolii ${ }^{20}$, L. Sanchez ${ }^{3}$, S. K. Solanki ${ }^{11,34}$, O. C. St $\mathrm{Cyr}^{14}$, T. Straus ${ }^{15}$, R. Susino ${ }^{5}$, L. Teriaca ${ }^{11}$,

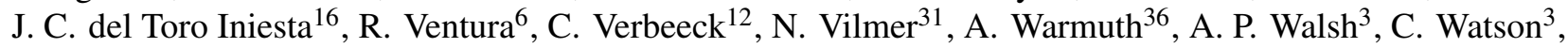
D. Williams ${ }^{3}$, Y. $\mathrm{Wu}^{1,36}$, and A. N. Zhukov ${ }^{12,28}$

\section{(Affiliations can be found after the references)}

Received 19 February 2019 / Accepted 13 July 2019

\begin{abstract}
Context. The Solar Orbiter spacecraft will be equipped with a wide range of remote-sensing (RS) and in situ (IS) instruments to record novel and unprecedented measurements of the solar atmosphere and the inner heliosphere. To take full advantage of these new datasets, tools and techniques must be developed to ease multi-instrument and multi-spacecraft studies. In particular the currently inaccessible low solar corona below two solar radii can only be observed remotely. Furthermore techniques must be used to retrieve coronal plasma properties in time and in three dimensional (3D) space. Solar Orbiter will run complex observation campaigns that provide interesting opportunities to maximise the likelihood of linking IS data to their source region near the Sun. Several RS instruments can be directed to specific targets situated on the solar disk just days before data acquisition. To compare IS and RS, data we must improve our understanding of how heliospheric probes magnetically connect to the solar disk. Aims. The aim of the present paper is to briefly review how the current modelling of the Sun and its atmosphere can support Solar Orbiter science. We describe the results of a community-led effort by European Space Agency's Modelling and Data Analysis Working Group (MADAWG) to develop different models, tools, and techniques deemed necessary to test different theories for the physical processes that may occur in the solar plasma. The focus here is on the large scales and little is described with regards to kinetic processes. To exploit future IS and RS data fully, many techniques have been adapted to model the evolving 3D solar magneto-plasma from the solar interior to the solar wind. A particular focus in the paper is placed on techniques that can estimate how Solar Orbiter will connect magnetically through the complex coronal magnetic fields to various photospheric and coronal features in support of spacecraft operations and future scientific studies.

Methods. Recent missions such as STEREO, provided great opportunities for RS, IS, and multi-spacecraft studies. We summarise the achievements and highlight the challenges faced during these investigations, many of which motivated the Solar Orbiter mission. We present the new tools and techniques developed by the MADAWG to support the science operations and the analysis of the data from the many instruments on Solar Orbiter. Results. This article reviews current modelling and tool developments that ease the comparison of model results with RS and IS data made available by current and upcoming missions. It also describes the modelling strategy to support the science operations and subsequent exploitation of Solar Orbiter data in order to maximise the scientific output of the mission.

Conclusions. The on-going community effort presented in this paper has provided new models and tools necessary to support mission operations as well as the science exploitation of the Solar Orbiter data. The tools and techniques will no doubt evolve significantly as we refine our procedure and methodology during the first year of operations of this highly promising mission.
\end{abstract}

Key words. Sun: corona - Sun: atmosphere - solar wind - Sun: general - Sun: fundamental parameters - Sun: magnetic fields

\section{General introduction}

Heliophysics is at the dawn of a golden age in observational capabilities. In addition to the on-going space missions like the Solar-TErrestrial RElations Observatory (STEREO; Kaiser et al. 2008), the SOlar and Heliophysics Observatory (SOHO; Domingo et al. 1995), the Solar Dynamics Observatory (SDO; Pesnell et al. 2012), Hinode (Hinode; Tsuneta et al. 2008a), and the Interface Region Imaging Spectrograph (IRIS; De Pontieu et al. 2014), space agencies are launching in addition to Solar Orbiter (Müller et al. 2019), described in detail here, the following two complementary missions: the Parker Solar Probe by NASA and PROBA-3 by the European Space Agency (ESA). Parker Solar Probe (PSP; Fox et al. 2016), which was launched on 12 August 2018, will, for the first time, measure the properties of the solar corona as close as nine solar radii from the solar surface. The PROBA-3 (Lamy et al. 2010; Renotte et al. 2016) will provide unprecedented white-light imaging of the 
low corona thanks to its revolutionary spacecraft (S/C) formation flying which reproduces the ideal observing conditions of solar eclipses. Data acquisition from the ground-based observatories such as the Global Oscillation Network Group (GONG; Hill et al. 1994; Harvey et al. 1996), GREGOR (GREGOR; Schmidt et al. 2012), and the Swedish 1-m Solar Telescope (SST; Scharmer et al. 2003); ground-based radio telescopes and neutron monitors spread around the globe; the Low-Frequency Array (LOFAR; van Haarlem et al. 2013) and the Daniel K. Inouye Solar Telescope (DKIST; Keil et al. 2011; Warner et al. 2018) will provide crucial complementary data. To take full advantage of this data so that we maximise our chances of answering the fundamental questions on the solar atmosphere, we need advanced data-analysis techniques and models capable of exploiting the multi-instrumental and multi-viewpoint data obtained by these observatories. The Modeling and Data Analysis Working Group (MADAWG) was created by ESA, in conjunction with two other working groups focused on the coordination of in situ (IS) measurements (Walsh et al. 2020) and remote-sensing (RS) observations (Auchère et al. 2020) to address these challenges head on, adapt existing tools and develop new ones to prepare scientific studies with Solar Orbiter and the future heliophysics observatories.

A majority of Solar Orbiter science goals rely on coordinated observations with multiple instruments (Müller et al. 2019), from IS instruments (Maksimovic et al. 2020; Horbury et al. 2020; Owen et al. 2020; Rodríguez-Pacheco et al. 2020) operating continuously along the orbit beginning in cruise phase, to the RS instruments (SPICE Consortium 2020; Solanki et al. 2020; Krucker et al. 2020; Antonucci et al. 2020; Rochus et al. 2019; Howard et al. 2020) observing during three 10-day windows per orbit called remote sensing windows (RSWs). The RSWs will typically be centred around perihelia as well as minimum and maximum heliographic latitudes. The operational constraints including limited telemetry due to orbital characteristics, variable data latency (up to 180 days) due to large variations in S/CEarth distance, and limited memory space on board motivated the need for long-term planning of top-level science operations (Zouganelis et al. 2020). The mission science outcome depends on coordinated observations of unpredictable solar activity. The high-resolution science requires fine-pointing to a target which position cannot be pre-planned. Moreover the nominal period of operation of Solar Orbiter is about seven years, hence covering more than half a solar activity cycle from solar minimum to maximum, the latter being currently expected to occur around 2024. This means that we can expect large variations of the activity level of the Sun during the nominal phase.

The science planning (Zouganelis et al. 2020) therefore folds in several time frames for mission level planning that depend on coordinated observations during opportunity windows along the trajectory, variable down link and limited memory on board as well as restrictions on RS observation time, telemetry management, and incompatibilities in electromagnetic cleanliness (EMC). The long-term plan ( $T-12-6$ months), medium-term plan ( $T-1$ month), short-term plan ( $T-1$ week) and very short term planning (VSTP) of science operations are described in the science planning article in the present volume (Zouganelis et al. 2020). During the latter VSTP, the high-resolution FOVs, that is PHI's High-Resolution Telescope (PHI/HRT; Solanki et al. 2020), the EUI High-resolution Imagers (EUI/HRI; Solanki et al. 2020) and the spectrometer SPICE (SPICE Consortium 2020), require fine-pointing to target while changing solar activity will impose pointing updates with short turn-around for data selection and calibration updates. To provide the necessary visibility, low-latency (i.e. quicklook) data will be required (Sanchez et al., in prep.).

Low-latency data, combined with modeling, can provide an important role for forecasting the location of targets and their magnetic connection with the S/C to choose targets and update the pointing. Once data has been acquired, models and tools will help interpret the daily low-latency data and decide which on-board data to select or prioritise for downlink (for those instruments with internal-storage, or those IS instruments using selective downlink capabilities). This is an important step to increase the likelihood of scientific studies that require combined IS-RS datasets. We described in this article the tools and models that we have put into place to help within this complex operational context.

Modeling also plays a central role for data analysis once the final data has been transmitted to the ground for the many reasons that are addressed in this article. Modeling compensates for an inherently incomplete sensing of the solar corona, can help connect different datasets and overall has become an essential tool to evaluate theories. At the time of writing this article we have not yet measured plasma IS in the corona inside 10 solar radii, solar plasma has been measured IS as close as $0.3 \mathrm{AU}$ and as far as the limits of the heliosphere. Yet the physical mechanisms that produce the heating of the corona, the formation of the solar wind, the acceleration of solar energetic particles and coronal mass ejections (CMEs) operate in the solar corona well inside 10 solar radii. To test the various theories and models proposed to exploit these phenomena, we need to retrieve the time-dependent evolution of the coronal plasma properties using different techniques supported by numerical models.

Our knowledge of solar plasma parameters is mostly inferred from RS observations from the optically-thick layers of the solar atmosphere to the more tenuous corona. Coronal observations are inherently $2 \mathrm{D}$ since radiation from the optically thin medium is integrated along the line of sight and techniques must be employed to retrieve its 3D structure. Due to the limited information on the parameters of the solar magneto-plasma, the exploitation of multiple instruments on different $\mathrm{S} / \mathrm{C}$ has become common practice in particular since STEREO. To achieve that and obtain a more complete picture of heliospheric phenomena, new techniques have been developed that integrate the information provided by instruments situated at multiple viewpoints (Sect. 2). Studies based on RS data ranging from radio to gamma ray energies permit detailed analysis of the energisation process of energetic particles, micro and macro-flares, and shock waves as well as studying the formation and acceleration of CMEs in the corona (see Sect. 2). Linking IS and RS data is a difficult task but some first important milestones have been accomplished with STEREO that we also discuss here. This motivates further developments in order to exploit the extraordinatary observational context that Solar Orbiter will bring as discussed in Sect. 3. Our knowledge of the magnetic field structure of the corona is also very incomplete since we measure the full 3D magnetic field only in certain regions of the photosphere and chromosphere. Reconstruction techniques and models must be used to retrieve the 4D structure of the quiet and transient corona (see Sect. 4), this is absolutely essential to make any kind of progress in our understanding of fundamental coronal processes. When connection can be established between particles and fields measured IS with their sources at the Sun, then a more complete picture of the physical processes coupling the solar corona to the heliosphere can be established (see Sect. 5). All these constitute the great challenges that we face to prepare the Solar Orbiter mission. 


\section{Heritage of the recent STEREO era}

The Sun-Earth Connection Coronal and Heliospheric Investigation (SECCHI; Howard et al. 2008) onboard the STEREO mission has provided since 2007, unprecedented imaging of solar storms from vantage points situated outside the Sun-Earth line. STEREO offered for the first time an uninterrupted imaging of plasma flows from the Sun to the Earth-like distances. The analysis of wide-angle imaging from multiple vantage points motivated the development of many algorithms and software tools in the last $10-15$ years. The tools primarily focus on deriving the 3D morphology of coronal and heliospheric structures, estimating their kinematics and direction of propagation in the corona and heliosphere. The following sections are purely intended to illustrate the power of using multiple viewpoints to infer the properties of coronal structures such as background streamers, coronal holes, CME flux ropes, shock waves. These techniques are being adapted and improved in preparation for Solar Orbiter as they have become integral parts of IS-RS studies (see Sect. 3).

\subsection{Locating coronal and solar wind structures in $3 D$}

To locate structures in the optically-thin corona, 3D reconstructions techniques constrained directly by coronal observations have been made freely available in the IDL Solarsoft distribution package, they include the basic potential field source surface (PFSS) model (e.g., Schrijver \& De Rosa 2003), software for 3D reconstructions of coronal loops (e.g., Aschwanden et al. 2009), flux ropes (e.g., Thernisien et al. 2009), and shocks (e.g., Vourlidas \& Ontiveros 2009; Kwon et al. 2014). These techniques deploy forward modeling approaches, where the user attempts to fit a geometric shape, such as an ellipsoid for a shock or a "croissant"-like structure for a flux rope, on snapshots of the transient in two or three simultaneous views.

The requirement of fitting the forward model in multiple views simultaneously tightly constraints the fit and results in quite robust reconstructions. To minimise the amount of free parameters, most models are rooted at Sun centre, rather than the source region (hence deflections are harder to simulate) and ignore small scale distortions on the modelled shape (hence local distortions of the structure are not modelled). Such models are generally better suited for examining the evolution of the large-scale envelope of transients and for investigating rotations or departures from self-similar expansion. More complex forward models have been used to account for distorted or irregular shapes of flux ropes and shocks (Wood et al. 2009) and for reconstructing (and locating the solar origin) of corotating interaction regions (CIRs; Wood et al. 2010) that were first identified in heliospheric images by Rouillard et al. (2008) and Sheeley et al. (2008). A simpler approach to 3D reconstruction is the localization in space of a single point in a structure via direct geometric triangulation, also referred to as tie-pointing. The accuracy of the technique relies on the ability to identify the same feature in images from two viewpoints, preferably taken at the same time. This is not always an easy task, either in the low corona, where features are small and can be obscured by the viewing geometry, or the outer corona, where the features are generally smoother and much more extended in space. An example and the issues behind tie-pointing in the outer corona are discussed in Liewer et al. (2011). The techniques work well for features with intrinsic small-scale structure, such as prominences (e.g., Panasenco et al. 2013; Thompson 2013) and comet tails (e.g., Thompson 2009). The tie-pointing routines are compatible with all WCScompliant FITS data and are available in Solarsoft.

\subsection{Feature tracking and trajectory analysis}

Other techniques are used to derive $3 \mathrm{D}$ trajectories and the kinematic properties of out-flowing features in the outer corona and inner heliosphere from height-time measurements of a feature. The techniques differ in their geometric assumption for transforming the observed elongation of the front (or other feature) to heliocentric distance. The most popular assumptions are: the point propagates radially (fixed- $\phi$ approximation; Kahler \& Webb 2007); the point lies at the Thomson Surface (point-P approximation); the point lies on the surface of an expanding sphere centered on the Sun (Harmonic Mean approximation; Lugaz et al. 2009); the point lies on the surface of a self-similarly expanding sphere propagating radially away from the Sun (SelfSimilar Expansion approximation; Davies et al. 2013). Obviously, all of these approximations make rather strong assumptions about the direction and shape of the feature in question. The resulting kinematics should be interpreted carefully, as discussed in the comparison study by Lugaz (2010), which suggest that the radial propagation appears to be the more robust approximation. However, these approaches have some advantages. The fixed-phi technique is thought to be most adequate to determine the trajectory of streamer blobs far out in the heliospheric and connect those with IS measurements (Rouillard et al. 2010a,b). They are easy and quick to calculate and can be applied to single viewpoint measurements (preferably from off the Sun-Earth line). A comprehensive application and comparison of these techniques in deriving the kinematic properties of CMEs in the inner heliosphere is given in Möstl et al. (2014). All these techniques have been integrated in J-map visualization tools available either in Solarsoft such as SATPLOT (see Sect. 6.5.1) or via interactive web-based interfaces such as the Propagation Tool (see Sect. 6.5.2: Rouillard et al. 2017).

Although these height-time measurements can be done directly in the coronagraphic or heliospheric images, the task becomes laborious as the contrast drops dramatically with increasing solar elongations. A much better approach is to transform the images in polar coordinates (elongation vs. polar angle) which increases the signal-to-noise ratio thanks to the angular sector averaging. Then a slice or band of pixels can be extracted at the desired polar angle, these slices can then be stacked in time to create an elongation-time map. These J-maps (Sheeley et al. 1999) can easily combine the slices from telescopes with different fields of view allowing the creation of elongation-time maps from the low corona to 1 AU (e.g., Sheeley et al. 2008; Davies et al. 2009). Propagating CMEs, but also smaller-scale features, such as streamer blobs, appear as distinct tracks of increasing elongation as a function of time. Their kinematics can be easily derived by fitting a variety of curves on these tracks based on the techniques introduced in the previous section. Crossing of tracks readily identify CME-CME or CME-CIR interactions. Diverging tracks in the low corona can mark the locations of collapsing loops such as those observed in the source regions of streamer blobs (e.g., Sanchez-Diaz et al. 2017a,b; Wang \& Hess 2018). Diverging tracks can also result from projection effects associated with radially out-flowing transients emitted by a single source region co-rotating away from the observer, these were recurrently observed in HI images taken by STEREO-B (e.g., Rouillard et al. 2010a). J-maps can also be used to connect, in a concise way, solar structures to their IS counterparts as illustrated in Fig. 1 (see review by Rouillard 2011 for more examples). Overall J-maps are extremely useful but must be analysed with care due to projection effects. 

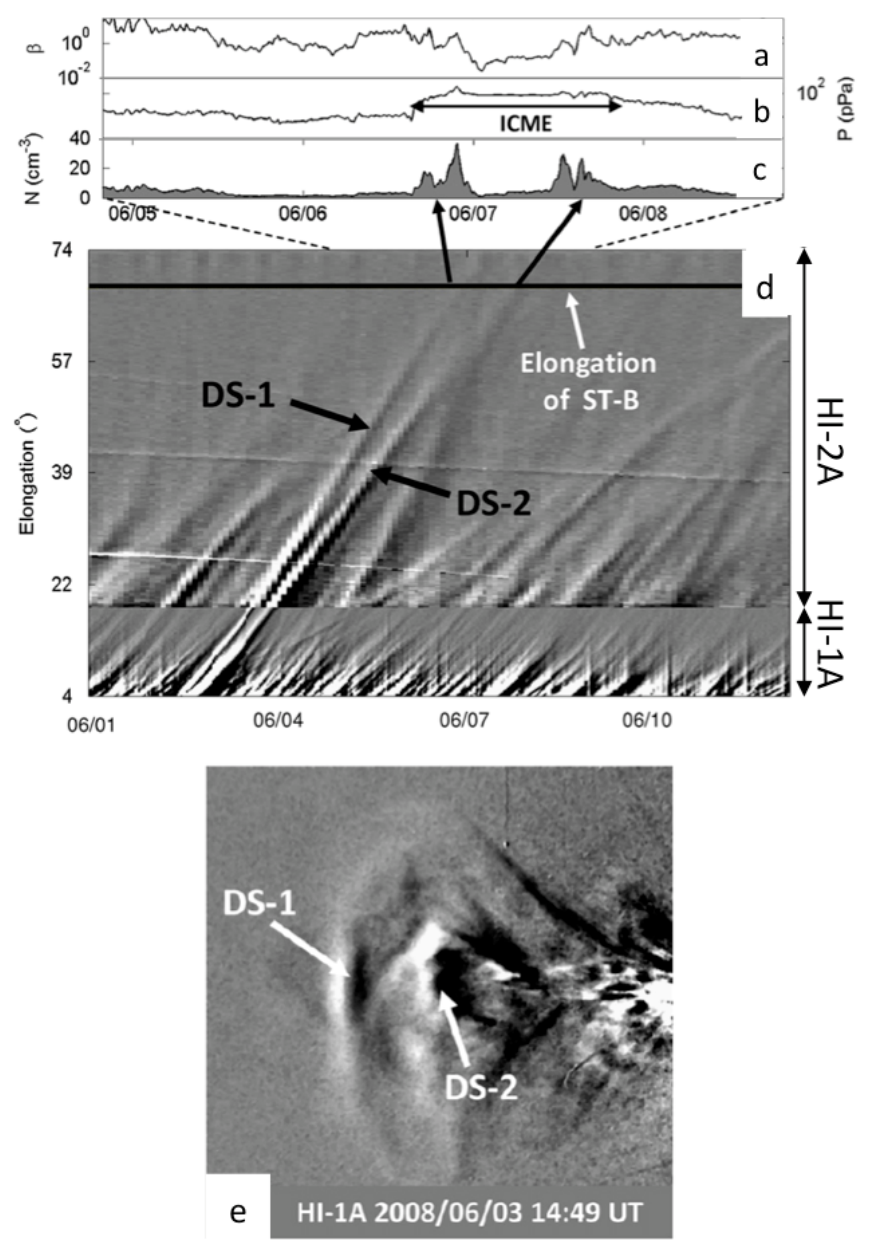

Fig. 1. Illustration of a successful linkage between a CME observed in white-light on STEREO-A on 3 June 2008 (panel e) with subsequent in situ signature at STEREO-B on 7 June 2008: the top panels show (a) plasma beta, $(b)$ total plasma pressure, $(c)$ number density from 2 to 7 June 2008 measured at STEREO-B. Middle: STEREOA elongation-time J-map combining HI-1 and HI-2 running difference images. The vertical dotted lines mark the extent of the ICME signature in the STEREO-B data. The ICME boundaries closely match the region bounded by the leading and trailing elongation-time tracks in the STEREO-A data (black arrows). Bottom panel: one of the HI$1 \mathrm{~A}$ running-difference images employed to build the J-map. From Rouillard (2011). Reprinted by permission of JASTP.

\subsection{Challenges faced so far when relating $R S$ with IS data}

We illustrate briefly three areas where good progress has been achieved in recent years by combining RS and IS using reconstruction and tracking techniques such as those described in the previous paragraph. This will motivate the next parts that present recent and upcoming developments to improve models and tools for Solar Orbiter science.

\subsubsection{Linking CMEs with Inter-planetary CMEs (ICMEs)}

Magnetometers onboard heliospheric observatories in the nearEarth environment sometimes display clear signatures of helical magnetic fields. A subset of those are called magnetic clouds (Burlaga et al. 1981) when a smooth and large magnetic field rotation is observed with associated drops in plasma beta and temperature. This helical topology is likely intimately linked to the initiation process of CMEs (e.g., Gosling 1990; Gopalswamy et al. 1998). RS detectors such as the coronagraphs and heliospheric imagers onboard STEREO or SOHO display change in the coronal structure with the appearance of bright features in white-light observations (e.g., Vourlidas \& Howard 2006; Webb et al. 2012). The observed patterns in the brightness of RS observations are studied as different CME components (Vourlidas et al. 2013). The core, usually observed remotely as a dark area is surrounded by a bright rim and is accepted to be the flux rope cavity. The IS signature of this cavity long suspected to be the magnetic cloud (Dere et al. 1999; Wood et al. 1999), was clearly identified as such using STEREO J-maps by Möstl et al. (2009) and Rouillard et al. (2009). The brightness in the front defines the leading edge associated with the sheath and interplanetary (IP) shock in the in situ observations (e.g., Kilpua et al. 2011).

Lynch et al. (2010) and the review by Rouillard (2011) (see Fig. 1) paint a rather positive picture for our ability to connect RS observations and IS measurements of certain interplanetary propagating (IP) structures, particularly under solar minimum conditions. The reality, however, is more complicated. Despite the availability of continuous imaging and tracking of IP disturbances from the STEREO heliospheric imagers, CME timeof-arrivals and especially speeds at $1 \mathrm{AU}$ can have errors upwards of $13 \mathrm{~h}$ and $200 \mathrm{~km} \mathrm{~s}^{-1}$, respectively, even for deprojected trajectories (Colaninno et al. 2013). Reconstructions of the CME shape from RS observations rarely agree with in situ ones (e.g., Nieves-Chinchilla et al. 2013; Wood et al. 2017). Some of the discrepancies can be attributed to limitations of the triangulation techniques (whether applied on J-maps or not) arising from the optically thin nature of the white light emission (Howard et al. 2012). Much of the remaining discrepancies, though, should arise from interaction between the structures and the ambient solar wind and/or from internal evolution that can lead to deflections (e.g., Isavnin et al. 2013, 2014), rotations (Nieves-Chinchilla et al. 2012) or distortions (Nieves-Chinchilla et al. 2013). We note, however, that these effects are inferred rather than observed directly in the data. In terms of the 3D global morphology, there is a current debate about distortion or deformation of the internal magnetic field configuration associated to the CMEs. The elongated dark cavity shape associated with the CME flux rope, called "pancaking", provides an evidence of a highly distorted global structure, probably due to the solar wind interaction (Odstrcil \& Pizzo 1999; Riley \& Crooker 2004; Owens 2006; Savani et al. 2010; DeForest et al. 2013; Owens et al. 2017). However, another interpretation is that it could be an effect of the projection of a 3D structure in the plane of the sky, creating the appearance of distortion (Vourlidas et al. 2011; Nieves-Chinchilla et al. 2013). In contrast, from the in situ perspective, the magnetic field strength asymmetries observed can be interpreted as a consequence of the local effect of flux rope expansion (Farrugia et al. 1993; Osherovich et al. 1993; Démoulin \& Dasso 2009) or erosion (Dasso et al. 2007; Ruffenach et al. 2012), but it could also be a result of the distortion (Hidalgo et al. 2002; Berdichevsky 2013; Nieves-Chinchilla et al. 2018a). Longitudinal deflections during inner heliospheric transit are particularly hard to quantify because heliospheric imaging is taken from and restricted along the ecliptic plane. This is the reason, for example, why the "garden-hose spiral" nature of solar wind streams has never been imaged directly but only inferred by de-projection techniques based on J-maps (Rouillard et al. 2008; Sheeley et al. 2008). While major progress was achieved with STEREO data, establishing the exact longitudinal extent of streamer blowouts (Vourlidas \& Webb 2018), whether CMEs deflect longitudinally or merge with each other, where CIRs form or how shocks evolve 
require observations away from the ecliptic (Gibson et al. 2018). This is an area where Solar Orbiter imaging, particularly from Solar OrbiterHI (Howard et al. 2020) will be trailblazing. However to fully exploit this data we need to adapt our techniques to account for rapidly varying vantage points located outside the ecliptic plane.

\subsubsection{Linking particle accelerators with SEPS}

The onset of large CMEs can suddenly transform the corona into an efficient particle accelerator. Solar energetic particle (SEP) events can be produced by CME-driven shocks or by other acceleration processes in association with flares. The many processes that can produce SEP events are not well understood and more importantly not very well distinguishable from particle measurements made at $1 \mathrm{AU}$. The link between solar storms (CMEs and flares), the perturbations of the corona they induce and the production of Solar Energetic Particles is a topic of active research. We briefly illustrate recent results that exploited RS-IS observations from multiple vantage points to investigate the link between CME substructures and SEPs. We highlight the challenges faced that have motivated the developments of new models and techniques for Solar Orbiter.

HELIOS measurements near 0.3 AU have revealed that a seemingly single gradual SEP event measured near $1 \mathrm{AU}$ can appear more complex at $0.3 \mathrm{AU}$ with the occurrence of multiple peaks in SEP fluxes suggesting that multiple events occurred successively. These multiple flux enhancements are presumably due to the changing conditions at the particle source that is magnetically connected to the $\mathrm{S} / \mathrm{C}$ but the propagation effects merge the events into one single long-lasting flux enhancement and decrease by $1 \mathrm{AU}$. This can either result from a changing magnetic connection to different particle accelerators or else from a single accelerator with strongly varying properties. It is therefore crucial to make measurements of energetic particles closer to the acceleration site so as to disentangle the properties linked to the acceleration site from the effects of particle transport.

The outer boundary of CMEs imaged in coronagraphs is the most likely location for shocks to develop as they mark the transition between the transient and the background corona. This region has therefore been the focus of many studies exploiting SOHO and STEREO data. The challenge resides in inferring when and where a shocks form on this boundary. Some early techniques derived, from a single viewpoint situated along the Sun-Earth line (SOHO), the properties of shocks driven by CMEs propagating in the plane of the sky (Bemporad \& Mancuso 2013). Improvements were brought by the application of 3D shock reconstruction techniques (see Sect. 2.1) to STEREO images from multiple viewpoints (e.g., Kwon et al. 2014; Rouillard et al. 2016). Important information on the formation and evolution of shock waves in the solar corona could then be obtained. Processing of these reconstructions provided the de-projected 3D expansion speed of the moving fronts in the corona for most CME directions (e.g., Rouillard et al. 2016), those speeds can be compared to the ambient Alfvén and magnetosonic speeds of the corona (provided by 3D models of the background solar corona) to derive the 3D Mach number of the entire moving shock. Recent studies have shown that pressure waves become shocks early on during the CME event (e.g., Rouillard et al. 2016; Plotnikov et al. 2017).

In order to compare derived shock properties with SEPs we need to determine how the shock connects magnetically to the $\mathrm{S} / \mathrm{C}$ making in situ measurements. This is likely the hardest and most uncertain step because, as already stated before, our knowledge of 3D magnetic fields both in the corona and in the inner heliosphere is uncertain. This point motivates the discussion in Sect. 5. The simplest approach typically used is to consider that the interplanetary magnetic field extending between a $\mathrm{S} / \mathrm{C}$ measuring SEPs and the upper corona is an Archimedean spiral defined by the plasma speed measured IS during the SEP. This spiral is traced down from the upper corona (typically at a source surface situated at $2.5 \mathrm{Rs}$ ) to the photosphere using a coronal model (see Sect. 4.1.1). The alternative is to use the results of full magnetohydrodynamic (MHD) models typically made available by numerical modellers via the Community Coordinated Modelling Center $\left(\mathrm{CCMC}^{1}\right)$. Comparative studies between shock waves and SEPs suggest a link between the strength of the shock quantified by the Mach number and the scattering-center compression ratio and the intensity of the SEP measured IS (e.g., Rouillard et al. 2016; Kouloumvakos et al. 2019). However to validate the shock hypothesis many questions must still be resolved concerning the timing, longitudinal spread and composition of SEPs and the relative role of shock waves and flares as particle accelerators (e.g., Lario et al. 2017). General conclusions from these recent studies highlight the importance of improving our knowledge of 3D plasma coronal parameters where the strongest particle acceleration is occurring and the absolute necessity to improve in our understanding of magnetic field connectivity between IS S/C and the low solar corona.

\subsubsection{Linking coronal plasma to the solar wind properties}

The solar magnetic field is known to also be the source of coronal heating, however how that field heats the plasma is still debated. A new generation of numerical models of the solar atmosphere that include both MHD and radiative effects (e.g., Hansteen et al. 2015) have shown that the continual re-configuration of chromospheric and coronal magnetic fields leads to the formation of current sheets supporting the idea of nanoflares as a driver of the heating process (e.g., Parker 1988). But we also know from high-resolution imaging and spectroscopy that the corona is permeated and traversed by MHD waves that may find ways to dissipate and heat the coronal plasma (e.g., De Pontieu et al. 2007). RS observations and theory have not yet revealed which of these mechanisms dominate the heating process or if they operate in conjunction.

Detection of the heated coronal plasma by IS measurements is mediated by the outflowing solar wind. Research over the last two decades (e.g., Hansteen \& Leer 1995; Cranmer et al. 1999; Lionello et al. 2009; Downs et al. 2010; van der Holst et al. 2014; Pinto \& Rouillard 2017; Usmanov et al. 2018) has shown that realistic coronal densities and solar wind mass fluxes can only be achieved with a detailed treatment of the thermodynamic processes throughout the computational domain from the upper chromosphere to the inner heliosphere. Thermal conduction is extremely important to transfer energy from the hot corona to the chromosphere that in turn enhances radiative processes (with its cooling effects) as well as chromospheric evaporation (and associated mass flows) feeding the solar wind. These energy exchange mechanisms are intimately linked to the distribution of plasma properties in the upper chromosphere and low corona. Knowledge of plasma densities and temperatures in these regions is therefore essential and in order to compare RS observations with model one must use realistic computations of the global magnetic field topology. Forward-modelling techniques can then be applied to confront model results to WL (white Light) or EUV imaging and spectroscopic observations

https://ccmc.gsfc.nasa.gov/ 
from the top of the chromosphere to the corona to test if the temperatures and densities are correctly reproduced by the model (e.g., Downs et al. 2010; van der Holst et al. 2014; Pinto \& Rouillard 2017). Assuming that heavy ions have the same temperature as the main plasma constituents and some typical coronal abundances, the brightness of the corona in EUV predicted by 3D models can be compared with EUV images from STEREO and SDO. Techniques that exploit images and spectroscopy have also been put in place to infer the distribution of coronal plasma temperature and speed (see Sect. 4.3) (e.g., Dolei et al. 2018) that can also be compared with coronal models. In this respect, white-light images are crucial to determine if the position and brightness of streamers and pseudo-streamers are correctly reproduced. Synchronous maps produced from several vantage points have become essential tools to help with these model evaluations as described in Sect. 4.2.

One step further is to consider the ionisation level of heavy ions. In recent studies RS and IS data have also been combined with numerical models to test solar wind models at heights where the ionisation level of heavy ions becomes frozen in (e.g., Landi et al. 2014). These studies compared the predicted coronal brightness of emission lines from carbon and oxygen ions derived from the output of different solar wind models (e.g., van der Holst et al. 2014; Cranmer et al. 1999) with spectroscopic observations. The predicted charge-state ratios were also compared with IS measurements. So far coronal models tend to under-estimate both the intensity of different emission lines and the charge state of different ions (Oran et al. 2015). This points to an inadequate modelling of ionisation processes such as the effect of non-thermal particles or the effect of differential particle flows. New global coronal models are currently being developed (including in the MADAWG) that fully couple the main and minor constituents and incorporate non thermal particles as well.

\section{Future opportunities to link Solar Orbiter RS with IS data to address some of the key science questions}

We briefly highlight here some examples of great opportunities that Solar Orbiter data will bring for future studies combining IS and RS data. This part introduces some of the technical challenges that we are currently facing to prepare for the arrival of these new datasets. The sections that follow present the solutions that the MADAWG is proposing to address some of these challenges.

\subsection{Investigations on the corona and the origin(s) of the solar wind(s)}

As introduced in the previous section, the chromosphere-corona and solar wind are a coupled system that must be modelled as such. In order to compare these global models with RS observations forward modelling has become a powerful approach. A forward modeling technique computes from a 3D data cube of plasma properties (derived by a model) a synthetic image or spectra for a vantage point by accounting for emission, absorption and scattering processes of electromagnetic radiation as self-consistently as possible. Forward modeling is an essential step at evaluating 3D coronal models close to the Sun in regions that even PSP will not measure directly. Coronal models can today be constructed using observed magnetograms (from MDI, HMI, and soon from the PHI instrument of Solar Orbiter, see Sect. 4.1.2) to produce a realistic distribution of open and closed field regions (Mikić et al. 2018; Réville \& Brun 2017). The FORWARD modeling package is the most complete package available in SolarSoft (Gibson et al. 2016). This tool produces images in most EUV and WL lines that will be obtained by Solar Orbiter and will have to be adapted to include the Solar Orbiter's fields of views. FORWARD has also become a central tool to compute synthetic spectro-polarimetric images comparable with current (CoMP) and future (DKIST) ground-based instrumentation. In addition, the CHIANTI database (Landi et al. 2013) can be used to produce synthetic brightness profiles in different emission lines typically observed in the chromosphere and corona in the X, UV and visible ranges. Creating synthetic profiles out of such models using CHIANTI or FORWARD, spectra and images will be compared to Solar Orbiter's unique RS data. Solar Orbiter's EUI, Metis, and Solar OrbiterHI will give us a new viewpoint to test background coronal models using forward modelling or image inversion (Sect. 4.3). Synthetic RS observations from the polar regions will be very useful at checking the relative locations of streamers and coronal holes against EUI and Metis images. We will finally understand how open and closed field lines interact due to their concomitant rigid and differential rotations.

As we discussed in Sect. 2.3.2 our ability to reconstruct the 3D properties of shock waves (Mach number, geometry, etc.) and to connect these properties with SEP measurements depend heavily on the accuracy of coronal models of both the plasma and magnetic fields. Once connection has been established between Solar Orbiter and its source region (a topic discussed in Sect. 5), comparison of model output in the form of time-series with the measured bulk solar wind parameters taken by the Solar Wind Analyser (SWA; Owen et al. 2020) are an obvious second step. Solar Orbiter will offer the unique capability of testing at the same time the bulk and composition of the source region of the different solar winds. Measurements from the Heavy Ion Sensor, part of the SWA suite, can help narrow the options for connectivity to the Sun. Charge-state ratios of heavy ions can be used to evaluate the level of heating near the source region and thus help distinguish among source region candidates (coronal hole vs. streamer) derived from modeling. In preparation for Solar Orbiter's comprehensive measurements of heavy ions, a new generation of multi-species coronal and solar wind models are being developed (e.g., Oran et al. 2015) that will enable detailed investigations of the composition of the coronal and solar wind plasmas. This includes investigations on the anomalously high abundance of elements with low First Ionisation Potential (the so-called FIP effect, e.g., Pottasch 1964; Fludra \& Schmelz 1999).

Solar Orbiter's SPICE instrument will produce maps of the relative abundance of heavy ions near the Sun that will be comparable with measurements by HIS, and will allow the testing of the solar wind models (Fludra \& Landi 2018). Comparison of charge states between HIS and SPICE will also be possible, though it will require additional information from a coronal electron temperature model, since SPICE measurements occur at altitudes below the freeze-in of charge states measured by HIS. Several charge state and elemental abundance ratios will be included in HIS low-latency data to facilitate these comparisons on the VSTP scale for science operations.

\subsection{Investigations on the global structure and origin(s) of CMEs}

Multiview and multipoint observations of the CME/ICMEs have established the basic framework for the understanding of the 
multitude of physical evolutionary processes that the CME undergoes in the interplanetary medium (see Manchester et al. 2017). The advent of STEREO has meant to be a driver for many studies but also uncovered many issues and discrepancies related with the initial CME stages and evolution throughout the interplanetary medium.

The techniques described in Sect. 2.2 are necessary when the RS observations are obtained from within the ecliptic plane and provide only direct information on the radial and latitudinal extent of out-flowing structures such as CMEs or CIRs. The EUI, Metis, and Solar OrbiterHI instruments will acquire coronal and heliospheric images from outside the ecliptic plane and therefore provide for the first time a direct view of the longitudinal and radial extent of solar features. Combined with images taken from inside the ecliptic plane by STEREO, SOHO or PROBA3 and PSP, the full 3D structure of CMEs, their flux ropes and shocks, will be derived with greater confidence. Currently, the debate about the CME global 3D morphology, magnetic field configuration, and implications in terms of heliospheric evolutionary physical processes is open. For instance, we currently have very little information on the structure of CME legs and how they slowly become part of the recently opened and closed solar magnetic flux. A polar view will provide valuable information on this transition especially combined with data from PSP much closer to the Sun.

$3 \mathrm{D}$ reconstruction techniques developed for STEREO Thernisien et al. (2009), Isavnin (2016) and Wood et al. (2017) are being adapted to include Solar Orbiter's fields of view and since the observation campaigns are rather short we should be able to produce catalogues of these 3D reconstructions to support research by the community. An illustration of future opportunities to reconstruct coronal shock waves using Solar Orbiter, STEREO, PSP and near-Earth orbiting S/C is shown in Fig. 2.

Following the path of current ICME catalogs from Wind, ACE or STEREO, Solar Orbiter suite of in situ instruments will be a valuable resource to identify ICMEs along the wide range of Sun distances of the mission. By using the models and techniques described by Nieves-Chinchilla et al. (2016, 2018b), the 3D reconstructions of the flux ropes inside ICMEs will be available for the community. Thus, for instance, this is a valuable resource to accomplish campaign event analysis when Solar Orbiter is at suitable conjunction with PSP, STEREO and/or near-Earth solar-heliospheric observatories (Wind, Advanced Composition Explorer, SOHO, DSCVR). The 3D in situ reconstructions are the more direct way to evaluate how the CMEs contribute to solar magnetic flux and helicity balance.

Trajectory analysis techniques require some deeper modifications. Indeed the slow motion of the STEREO S/C meant that a CME moving outwards outside the ecliptic plane remained at fairly constant position angle throughout its transient to $1 \mathrm{AU}$. This is no longer the case with Solar Orbiter or PSP and new techniques are currently being considered that rely heavily on forward modelling, integrating self-consistently the changing viewpoint of the probes and the scattering/emission processes. Estimated trajectories will allow us to test full MHD propagation models as such as the EUHFORIA model (see Pomoell \& Poedts 2018 and Sect. 5.3). They will allow us to refine our understanding as well as our predictive skills of propagating CMEs, especially during the crucial first phases of evolution after their onset.

\subsection{Investigations on the properties and origin(s) of SEPS}

The variability of SEPs suggests rapid changes in the properties of the accelerator and the magnetic connectivity of that acceler-

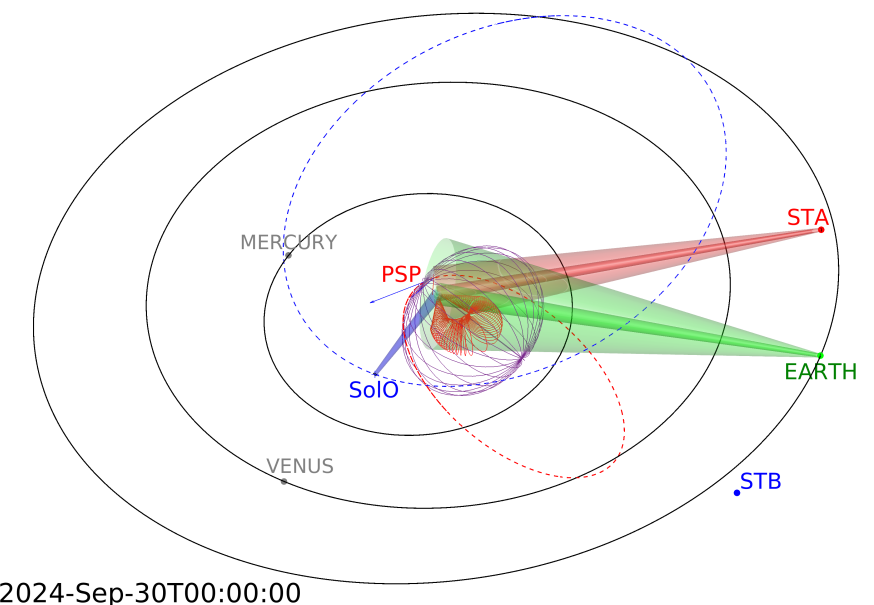

Fig. 2. A schematic to illustrate the possible synergies between missions located in the inner heliosphere on 30 September 2024. The figure shows the relative positions of the planets as well as the Sun, SOHO, Solar Orbiter, PSP and STEREO-A. The fields of view of a subset of the instruments onboard these missions is also shown (SOHO: $\mathrm{C} 2 / \mathrm{C} 3$, STEREO: COR-1 and COR-2, Solar Orbiter: Metis). In addition the 3D structure typically used to fit a flux rope and a shock in 3D are also shown.

ator to the point of IS measurements. To test further the shock acceleration scenario we must pursue modelling of the evolution of shock waves as accurately as possible to enable a complete modelling of particle acceleration with realistic shock parameters Afanasiev et al. (2018). Since the number of fast CME events that Solar Orbiter will observe during RSWs will be rather limited, catalogs of triangulated CME shock waves that integrate Solar Orbiter data will also be produced and made available to the community. In addition the proximity of Solar Orbiter to the corona means that standard techniques to derive the release time of SEPs measured by Solar Orbiter such as velocity dispersion analysis and spectra of the different SEP events may be more accurate than those produced near $1 \mathrm{AU}$ and can also be made available to the community.

Better coronal models will improve our computations of shock properties and provide more reliable estimates of the magnetic connectivity between the shock and the energetic particle detectors Rodríguez-Pacheco et al. (2020). To this end, the 3D shock triangulation codes (e.g., Kwon et al. 2014) readily available in SolarSoft and the Coronal Analysis of SHocks and Waves (CASHeW) framework (Kozarev et al. 2017), compatible with SolarSoft routines will be modified to naturally include these new viewpoints. Remote observations from Solar Orbiter will also enable a more accurate geometrical characterization of coronal and interplanetary shocks, so that non-radial propagation and small-scale structure of the fronts may be accounted for. Current efforts to prepare for Solar Orbiter data exploitation include the coupling of particle acceleration based on the theory of diffusive-shock acceleration and shock-drift acceleration to the 3D shock modeling (e.g., Afanasiev et al. 2018).

In the case of flare accelerated SEPs, the location of the acceleration site in the solar atmosphere could be identified using the X-ray signature that particle travelling down to the collisional atmosphere will emit through interactions with the dense solar surface. Full 3D modelling of plasma heating and particle acceleration on flares will be invaluable in identifying the links between the emission diagnostics that will be detected by STIX (Krucker et al. 2020) and EUI (Rochus et al. 2019). 
The detection of flare accelerated particles close to the Sun will significantly reduce the influence of particle transport on estimates of the particle travel time and then on the particle escape time. The in situ detection with EPD of energetic electrons with temporally correlated radio type III observations with RPW (Maksimovic et al. 2020) and ground-based observatories (in the case when they observe the same portion of the solar disk), and hard X-ray flare observations with STIX will allow the electron solar source region to be located without ambiguity and the magnetic connectivity to the $\mathrm{S} / \mathrm{C}$ be well established. A more precise timing and association between IS energetic electrons and X-ray emitting electrons will allow in particular to re-investigate the relationship between the in situ electron spectra and the electron spectra at the sun deduced from X-ray observations (see e.g., Krucker et al. 2007). In this respect, new models have been developed in recent years that couple the large-scale evolution of magnetic fields with the acceleration of particles and the injection of these particles into the open magnetic field (Snow et al. 2017).

\subsection{Investigations on the origin of the solar magnetic field}

The solar magnetism originates from dynamo action in the solar interior within and at the base of its convective envelope. The most realistic solar dynamo models today compute selfconsistently the complex and non-linear interplay between convection and magnetic field. They generally rely on a combination of physical mechanisms such as: strong shear in the tachocline and throughout the convective envelope (the Omega effect), vortical inductive turbulent motions (the alpha effect), advection of the magnetic flux by the meridional circulation, and emergence of magnetic structures through the photosphere (the BabcockLeighton effect). Observations of the photospheric magnetic field of the Sun are extremely valuable for our understanding of the internal processes sustaining the solar magnetic field, along with helioseismic constraints. In particular, the detailed knowledge of the magnetic properties of the Sun at high latitudes (magnetic flux transport, strength of the polar field) is of paramount importance because they will allow to better characterise the intricate process of global polar field reversal.

Solar Orbiter, and in particular the PHI instrument will shed new lights on these processes. Observations of the Zeeman effect at the pole will provide valuable insights on how the flux cancels out when the solar magnetic field reverses, what is the relative importance of the small scale magnetic flux near the pole (Tsuneta et al. 2008b; Shiota et al. 2012), and how ultimately the poloidal field is regenerated (Benevolenskaya 2004). Furthermore, new constraints on the patterns of the differential rotation and meridional circulation at the pole will be obtained through correlation tracking of small features, direct imaging of Doppler shifts and helioseismic observations from a high latitude vantage point. This will provide critical inputs for dynamo models that are generally sensitive to the flows and their structure at high latitude (Charbonneau 2010). The deeper structure of the flows sustaining the solar dynamo down to the solar tachocline will also be probed with more accuracy thanks to stereoscopic helioseismic observations combining Solar Orbiter and, e.g. SDO and GONG. Altogether more precise constraints on the flows and field of the Sun will help disentangle whether separate dynamo processes are acting in our star, by leveraging these new key observations from Solar Orbiter in 2D and 3D mean-field (e.g., Charbonneau 2010; Yeates \& Muñoz-Jaramillo 2013; Karak \& Miesch 2017), 3D turbulent small-scale (e.g., Vögler \& Schüssler 2007), and 3D large-scale (e.g., Brun et al.

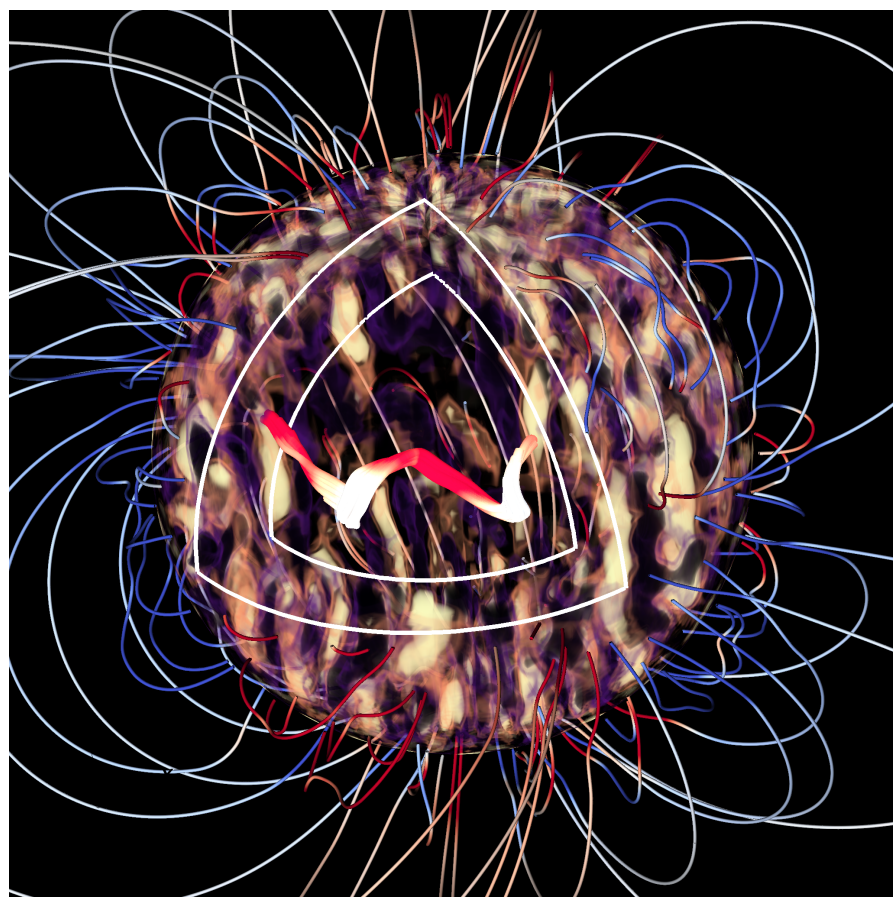

Fig. 3. Non-linear, 3D cyclic dynamo in global turbulent simulations of Strugarek et al. (2017). The rising/sinking convective cells are shown by the dark/bright patches in the convective envelope. A quadrant of the sphere has been cut out (white lines) to reveal the self-consistent generation of strong toroidal magnetic field (red-white ribbon) at the base of the simulated convection zone. A potential extrapolation of the magnetic field outside the star is shown with red/blue tubes labelling magnetic field lines exiting/entering the solar surface.

2015; Strugarek et al. 2018) models of the solar dynamo. An example of such models can be found in Fig. 3 where a solarlike cyclic dynamo seated deep in the convective envelope of the star was shown to occur due to the non-linear interaction between the internal flows and the dynamo-generated large-scale magnetic field (Strugarek et al. 2017). Solar Orbiter will further bring important constraints to resolve the so-called spot-dynamo paradox, that is to understand whether magnetic spots are a simple manifestation of the deeply-seated solar dynamo, or play an active role in the dynamo allowing a cyclic behaviour of the solar magnetic field (Brun \& Browning 2017).

Finally, Solar Orbiter will also provide important insights for the prediction of the solar cycle and solar activity. Indeed, Cameron \& Schüssler (2015) and Nagy et al. (2017) have showed that the surface properties of the solar magnetic flux has a strong impact on our ability to predict the strength of the next solar cycle, because e.g. of the possible emergence of "rogue active region" late in the cycle phase. Such observational constraints from various vantage points are needed today in order to be used in data-assimilation models to predict the length and strength of the upcoming solar cycle (e.g., Petrovay 2010; Hathaway \& Upton 2016; Hung et al. 2017; Jiang et al. 2018).

\section{Techniques and tools to restore the 4D corona}

As discussed repeatedly in the previous parts, scientific progress depends critically on our ability to model the magnetic and plasma properties of the background plasma. The effort of the community towards such improvements is discussed here. 


\subsection{Inferring the $3 D$ coronal magnetic field}

The magnetic field is the key player in most (if not all) processes taking place in the solar corona. It permeates all layers of the solar atmosphere, controls the flow and dissipation of energy, and shapes the coronal structures. We discuss in the next sections the challenges faced when modelling the 3D magnetic field.

\subsubsection{Some challenges to model coronal magnetic fields}

The magnetic field is difficult to measure in the corona because it is weak and has a complex topology in most cases. Furthermore, the solar corona is optically thin at most wavelength ranges (visible, UV, and EUV) that are relevant to the measurement of the magnetic field.

Several methods show great promise for measuring the field in the low corona and complement each other. They are the Zeeman and Hanle (Hanle 1924) effects and the radio gyroresonance and bremsstrahlung. Radio gyroresonance and bremsstrahlung provide the magnetic field strength and the line-of-sight [LOS] component above on-disk ARs, respectively. Iso-Gauss surfaces can be inferred from the radio emissions but their heights in the solar atmosphere remain ambiguous (Zlotnik 1968; White \& Kundu 1997; Lee et al. 1997, 1998; Brosius et al. 1997). Raouafi $(2005,2011)$ provide comprehensive reviews on the different methods used or that can be used to measure the magnetic field directly in the solar corona.

The Zeeman and Hanle effects have been used to obtain diagnostics of the coronal magnetic field (Harvey 1969; Casini \& Judge 1999; Raouafi et al. 1999, 2002; Raouafi 2002). In the near future, groundbased observatories will be key to exploring the low corona through direct polarimetric diagnostics of the magnetic field using the Zeeman and Hanle effects. We expect the DL-NIRSP and Cryo-NIRSP instruments of the 4-meter DKIST (Keil et al. 2011) to provide routine measurements of the coronal field using these two mechanisms. Other telescopes, such as the COronal Solar Magnetism Observatory (COSMO; Tomczyk et al. 2016), will also contribute significantly. It is now clear that measuring magnetic field in the corona is a prerequisite for understanding global phenomena such as space weather. Ground measurements alone may not be sufficient and the need for a space mission dedicated to this goal is now very obvious.

The Zeeman effect produces a frequency-modulated polarization signal, which is sensitive to both the direction and strength of the magnetic field. Achieving accurate measurements of the coronal magnetic field through the Zeeman effect is difficult, however, because the magnetic field is weak (typically of the order of $100 \mathrm{G}$ in the strongest field regions above the photospheric imprint of solar active regions), the Zeeman splitting scales with the wavelength squared, and emission lines have large Doppler widths (Bommier \& Sahal-Brechot 1982; Raouafi et al. 2016). Coronal magnetic diagnostics through the Zeeman effect are thus best achieved with infrared spectral lines (Harvey 1969; Kuhn et al. 1996; Judge 1998). More accurate measurements have been achieved by Lin et al. $(2000,2004)$ using the Fe XIII $10747 \AA$ line to measure fields at heights ranging between 1.12 and $1.15 R_{\odot}$ from sun center. This type of measurements of coronal field strength via the Zeeman effect suffered from very poor spatio-temporal resolution that is necessary to collect sufficient signal in the very weak circular polarization profile. Even at these wavelengths the fraction of circular polarization is only expected to be of the order of $10^{-4}$ (Querfeld 1982; Plowman 2014). In such circumstances, measuring circular polarization associated with a 1 Gauss magnetic field in $15 \mathrm{~min}$ with a 2 arcsec resolution requires larger aperture telescopes, such as the Large Coronagraph $(1.5 \mathrm{~m})$ on COSMO and DKIST.

On the other hand, the Hanle effect is a modification of the linear polarization of a spectral line in presence of a magnetic field (Hanle 1924; Charvin 1965; Sahal-Brechot et al. 1977). Contrary to the Zeeman effect, the Hanle effect requires the prior existence of polarization through other physical processes such as radiation scattering. Depending on the choice of spectral line and the direction and strength of the magnetic field, the Hanle effect can provide measurements of magnetic fields from a few milli-Gauss to several hundred Gauss (Bommier \& Sahal-Brechot 1982; Raouafi et al. 2016). The Hanle effect can therefore be a very powerful tool for the diagnosis of coronal magnetic fields. This has been confirmed both theoretically and observationally with, e.g. off-limb observations of the infrared Fe XIII lines (see Sect. 4.1.3). Unfortunately, current routine coronal polarimetry is limited to the Fe XIII lines for which the Hanle effect operates in the saturated regime (Casini \& Judge 1999; Rachmeler et al. 2013). The latter implies that the linear polarization signal is sensitive to the magnetic field direction but not its strength. Polarization measurements with other spectral lines (e.g the infrared He I $10830 \AA$ or the UV H I Ly $\alpha$ lines) will be required to circumvent that issue (e.g., Raouafi et al. 2016). In the UV wavelength range, SOHO/SUMER provided a unique measurement of the linear polarization of the O VI $1032 \AA$ line (Raouafi et al. 1999). Raouafi et al. (2002) showed that the SUMER measurements can be interpreted as the signature of the Hanle effect due to the coronal magnetic field.

Even in the best conditions (e.g. choice of spectral lines, large aperture telescopes), determining the 3D solar magnetic field from coronal polarimetry remains a real challenge. Indeed, the solar corona is an optically thin plasma at most wavelengths. Therefore, whether the polarization signal is due to Zeeman, Hanle, or some other mechanism, the signal is the integration of all the plasma emission along the line of sight. As a consequence, it is very difficult to obtain individual coronal magnetic field data at specific positions along the line of sight without stereoscopic observations (Kramar et al. 2013, 2016). Our knowledge of the 3D coronal magnetic field therefore relies on 3D modeling from photospheric and/or chromospheric surface measurements.

The most widely used numerical models that exploit surface vector magnetograms broadly fall into two categories: magnetostatic (MHS) and MHD models. The former includes both the force-free field (FFF) models (including potential and currentcarrying magnetic fields) which do not consider the plasma and where the electric currents are parallel to the magnetic field lines (e.g., Wheatland et al. 2000; Wiegelmann 2004; Valori et al. 2005; Amari et al. 2006; Contopoulos et al. 2011; Malanushenko et al. 2014), and magnetostatic models which do (Low 1992; Aulanier et al. 1999; Wiegelmann \& Neukirch 2006; Wiegelmann et al. 2007; Zhu \& Wiegelmann 2018). Such magnetostatic solutions are used as initial states to the magnetohydrodynamic models (Mikić et al. 1999; Inoue et al. 2011; Feng et al. 2012; Zhu et al. 2013). Both these categories include local, i.e. limited to single active regions or to active region groups (e.g., Canou et al. 2009; Gilchrist et al. 2012; Jiang et al. 2014), and full Sun global models (e.g., Titov et al. 2011; Platten et al. 2014; Tadesse et al. 2014; Yeates et al. 2018). In practice, local current-carrying force-free, MHS and MHD models better account for the strong electric currents developing in the vicinity of active regions and are therefore better suited to model their complex magnetic field. Global models provide a full Sun 3D coronal magnetic field that allows to better describe the effects of remote connections between active 
regions and with the solar wind, and can be used to derive the location and distribution of the suspected source regions of the solar wind (such as helmet streamers, pseudo-streamers, coronal holes). That being said, recent developments have been made to produce global models that also allow to account for the strong electric currents in active regions (e.g., Amari et al. 2018).

Despite all these models, there is currently no unique solution of the 3D coronal magnetic field derived from the photospheric measurements. Apart from the fact that such models are built from different assumptions and numerical techniques that inherently result in differences in the final solution, some observational limitations further enhance the lack of ground truth 3D coronal magnetic field. First, photospheric measurements of the transverse magnetic field, which allow to derive the photospheric vertical electric currents, are subject to the $180^{\circ}$ ambiguity (Harvey 1969; Metcalf et al. 2006; Leka et al. 2009). Different methods lead to different ambiguity removal, and hence, different boundary conditions. Second, existing MHS and MHD models do not use the photospheric vector magnetic field in the same manner (e.g., partial vs. full vector, pre-processing for currentcarrying FFF models; see e.g., De Rosa et al. 2009), also leading to different boundary conditions. How the boundary conditions deduced from a simulated Solar Orbiter's PHI instrument influence the quality of 3D magnetic field models has been investigated in Wiegelmann et al. (2010). Third, global models lack of proper boundary conditions. In particular, they require the simultaneous full $4 \pi$ sr measurement of the photospheric vector magnetic field, which is not possible from the single vantage point given by Earth. Full $4 \pi$ sr vector magnetograms must therefore be built from non-simultaneous photospheric measurements such as, e.g. synoptic maps (see Sect. 4.1.2). However, this can have strong effects on global reconstructions of the magnetic field because changes occurring on the invisible or poorly observed surface of the Sun can cause strong changes in the global topology of the corona. All these issues directly affect our ability to infer the connectivity of a $\mathrm{S} / \mathrm{C}$ to the regions where the solar wind forms, as well as to determine whether or not there is a universal threshold for solar flares and CMEs and thus predict them.

For the first time, the Solar Orbiter's PHI instrument (Solanki et al. 2020) will provide surface magnetic field measurements from outside the Sun-Earth line and from outside the ecliptic plane. Despite possible issues with cross-calibration of magnetic field measurements between different instruments and observatories (see e.g., Bai et al. 2014; Riley et al. 2014; Watson et al. 2015), coordinated observations from Earth and Solar Orbiter's PHI will provide better photospheric boundary conditions for 3D modeling. For instance, observations of the same solar region from both Solar Orbiter's PHI and Earth's orbit (via, say, $\mathrm{SDO} / \mathrm{HMI}$ ) can enable the unique removal of the $180^{\circ}$ ambiguity in the transverse component of the photospheric magnetic field (Fig. 4, see Sect. 4.1.2). Solar Orbiter's PHI vantage point will further provide larger simultaneous photospheric spatial coverage for global models, reaching its maximum when at $180^{\circ}$ from the Sun-Earth line (although this will be accompanied with the resurgence of the $180^{\circ}$ ambiguity problem). PHI will also allow larger temporal coverage of active regions observed from Earth, providing more complete timeseries of photospheric vector magnetograms for data-driven models of the 3D coronal magnetic field. Finally, EUV data of coronal loops from EUI will provide more information on the shape of magnetic field lines to help better constrain 3D magnetic field models (e.g., Malanushenko et al. 2012; Aschwanden 2013). A novel approach, dubbed Non-linear Force-free Coronal Magnetic Stereoscopy is to combine mag-

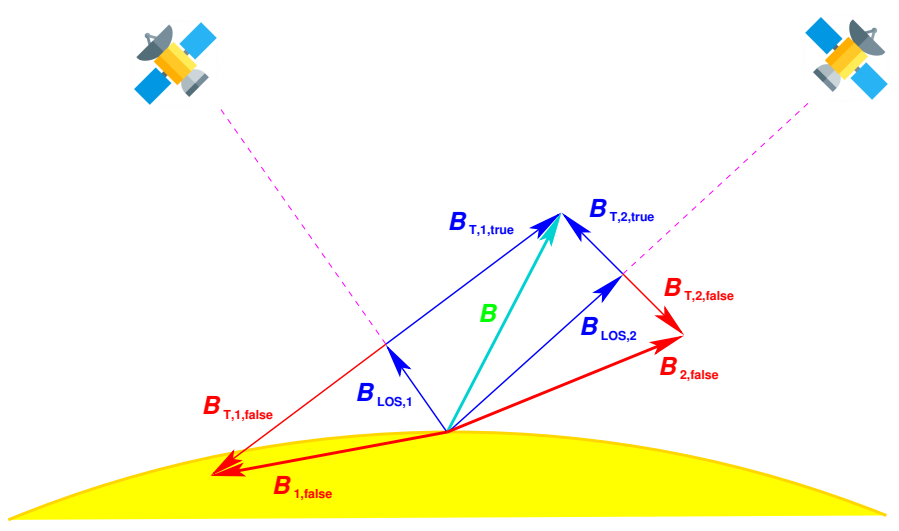

Fig. 4. Sketch of the ambiguity arising by deriving the magnetic field vector using the Zeeman effect. Observations from a single vantage point allows for two different solutions $\boldsymbol{B}$ or $\boldsymbol{B}_{1, \text { false }}$. Stereoscopic observations make possible the selection of the correct solution between $\boldsymbol{B}$, $\boldsymbol{B}_{1, \text { false }}$ and $\boldsymbol{B}_{2, \text { false }}$.

netic field extrapolations from vector magnetograms with stereoscopy from EUV-images (see Chifu et al. 2015, 2017). Using additional observations from out of the ecliptic by EUI will be of great benefit to improve both the stereoscopic 3D-reconstruction of loops and constrain the magnetic field models.

\subsubsection{Opportunities to improve magnetograms with Solar Orbiter}

Nearly all coronal models use global maps of photospheric magnetic field distribution as their primary input. For decades diachronic synoptic maps were typically used as input to coronal models, but now the heliospheric community is moving towards using synchronic maps that attempt to estimate the global magnetic field distribution at any moment in time (see, e.g., Ulrich \& Boyden 2006; Riley et al. 2014). Diachronic maps are often constructed by extracting bands of pixels situated along or near the central solar meridian imaged by a magnetograph, and as the sun rotates, the bands are then stacked as a function of time (or longitude) over a solar rotation. Another common approach is to use a longitudinal weighting function that is heavily weighted at the central meridian of each magnetogram added. Diachronic maps thus represent the time history of the central meridian of the Sun over a solar rotation. Synchronic maps attempted to represent the instantaneous state of the Sun's global surface field by using flux magnetic flux transport processes (e.g. Worden \& Harvey 2000; Schrijver \& De Rosa 2003; Arge et al. 2013; Upton \& Hathaway 2014; Hickmann et al. 2015). To account for the inherent uncertainty with flux transport modeling (e.g., such as the uncertainties in the meridional drift or different rotation rates or lack of observations on the solar far-side), the Air Force Data Assimilative Photospheric flux Transport (ADAPT) model utilizes an ensemble (with typically 12 realizations) of synchronic synoptic maps (based on Worden \& Harvey 2000) and state-ofthe-art data assimilative techniques (see, Hickmann et al. 2015) to represent as realistically as possible the spread in the uncertainty of the state of the global photospheric magnetic field (Arge et al. 2010). The ADAPT global magnetic maps, using the available Earth/L1 perspective magnetograms, are publicly available ${ }^{2}$ and used within the heliospheric modeling community, e.g., for time-dependent MHD simulations of the inner heliosphere

\footnotetext{
2 https://www.nso.edu/data/nisp-data/adapt-maps/
} 
(Merkin et al. 2016) and ensemble modeling of the large CME during July 2012 (Cash et al. 2015). In addition, ADAPT forecast maps are utilized to predict the observed F10.7 values (i.e., the solar radio flux at $10.7 \mathrm{~cm}$ ) and bands within the VUV (vacuum ultraviolet, between 0.1 and $175 \mathrm{~nm}$ ) solar irradiance (Henney et al. 2015). ADAPT maps are also integrated in the operational support for the Solar Orbiter mission (see Sect. 5).

The origin of magnetic forecast maps is given by single photospheric magnetic field maps of the solar disk. Photospheric vector magnetic field maps obtained from a single vantage point are, however, strongly affected by the $180^{\circ}$ ambiguity of the transverse Zeeman effect which leads to an ambiguity of the obtained field azimuth with respect to the line of sight and to a big uncertainty of the actual field strength (and even polarity) when transformed to the local, heliographic coordinate system. As only two solutions are possible, observations of the same solar region from another vantage point provide the unique opportunity to resolve this ambiguity (see Fig. 4).

Coordinated observations between PHI and, e.g., HMI onboard SDO (see Scherrer et al. 2012) will lead to new methods to produce more realistic vector-magnetograms. Combining observations from two vantage point requires, however, new developments in order to overcome the obvious obstacles for a successful application of this technique. As shown in Fig. 5 the primary obstacle is the geometric foreshortening and the necessity to cross-correlate observations from different instruments which will be affected by different geometric distortions, spatial resolution and noise levels which becomes even more relevant as the spatial resolution of PHI changes with Solar Orbiter's orbital position. PHI will sense this effect for the first time in the history of solar magnetography. Although PHI and HMI obtain their magnetic fields from observations of the same spectral line (Fe I $6173 \AA$ ) the different spectral resolution and heliocentric angles provide observations from different atmospheric heights. As it is well known that the magnetic field structure of the photosphere changes rapidly with height (see e.g. Rempel \& Cheung 2014) a careful consideration of this effect is essential and the final goal of such a development must be a combined stereoscopic inversion of the radiative transfer equation of both observations (i.e. using data from the PHI raw data observation mode) which intrinsically consider the different formation heights. A comprehensive space-based study of azimuth-disambiguated electric current density from two different angles and spatial resolutions will also provide unprecedented clues toward both understanding solar photospheric magnetism and extrapolating the photospheric boundary by any modeling means: magnetostatic, magnetohydrostatic, or magnetohydrodynamic (see, e.g., Georgoulis 2018).

Figure 5 was obtained by synthesizing the Fe I $6173 \AA$ spectral line from a realistic magnetohydrodynamic model of a solar region (see Riethmüller et al. 2017, for details) and for two different heliographic angles. The thus obtained solar spectra have been fed to the PHI instrument simulator (SOPHISM, see Blanco Rodríguez et al. 2018), assuming PHI-HRT observations at $0.28 \mathrm{AU}$ solar distance, and a subsequent inversion of the radiative transfer equation has been carried out with the SPINOR code (Solanki 1987; Frutiger 2000). As the resulting maps of the magnetic field strength are quite comparable the field azimuth already hints to rather different solutions in the two models, e.g. at the left small pore at $y=200$. More detailed studies, e.g. including also instrumental parameters of HMI, have to be developed prior to establish a tool to stereoscopically resolve the $180^{\circ}$ ambiguity.
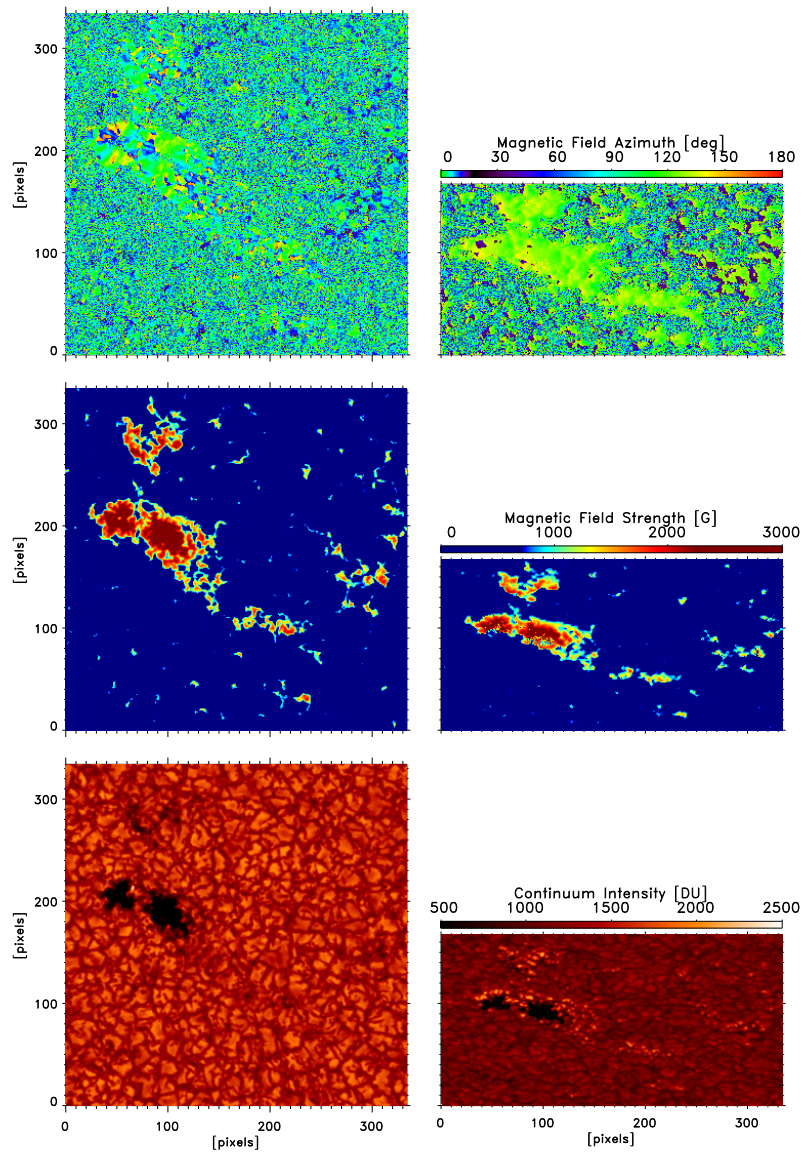

Fig. 5. MHD simulation of a realistic active region as seen at disk ceter (left panels) and at a heliocentric angle of $\theta=60^{\circ}$ (right panels). The synthesized spectra have been degraded using the instrumental parameters of the PHI-HRT telescope at a solar distance of $0.28 \mathrm{AU}$ and subsequently inverted.

It is also well known that polar regions play a key role for the progression of the solar cycle and, likely, for the underlying dynamo mechanisms (see Sect. 3.4 and, e.g. Charbonneau 2010; Cameron \& Schüssler 2015; Petrie 2015). Solar dynamo models, therefore the testing of solar dynamo models, therefore, notably depends on improved vector magnetic field maps of the polar regions. And consequently, also do models that link solar dynamos to the properties of the 3D corona and wind (Pinto et al. 2011; Kumar et al. 2018).

Löptien et al. (2015) list the required observation campaigns and analysis tools allowing to better understand the dynamics of the surface and subsurface layers of the polar regions with measurements carried out by Solar Orbiter. Of particular interest is the latitudinal dependence of the large and small scale magnetic field structures and their temporal evolution in order to disentangle contributions from global and local dynamo actions to the global solar magnetic field. In addition, the magnetic helicity, as an invariant of ideal magnetohydrodynamics, is assumed to denote a quantity which directly measures the properties of the solar dynamo. Solar Orbiter will allow us to generate improved helicity spectra and their temporal evolution along the solar cycle since during high latitude phases the obtained vector-magnetograms will be less dominated by active regions and false polarity detection because of the $180^{\circ}$ azimuth ambiguity. In order to benefit from the inclined orbit, a study will be performed to determine whether the helicity spectra can be 
retrieved from the standard PHI data products or if downloading raw data (Stokes parameters) is required. Ideally, this study will define a dedicated PHI operation mode to compute the helicity spectra onboard PHI as this instrument produces also its standard data products (cf. Albert et al. 2018). Overall it is indeed crucial to link magnetic flux emergence on all scales and latitudes on the solar surface to the internal dynamo mechanism and how these properties vary along the 11-yr cycle, in order to understand how the solar dynamo works in details. Solar Orbiter's unique orbital trajectory jointly with PHI data measurements will provide such constraints.

\subsubsection{Combining surface magnetograms with off-limb coronal polarimetry}

Polarimetric measurements in the corona through the Zeeman and Hanle effects (described in Sect. 4.1.1) allow us to diagnose the strength and direction of the coronal magnetic field. Coronal polarimetry has received more and more attention in the last decade. The Coronal Multichannel Polarimeter (CoMP; Tomczyk et al. 2008) contributed to this field by providing offlimb coronal emission-line polarization measurements in the infrared through the Fe XIII 10747 and $10798 \AA$ lines. Such measurements have been obtained daily since 2011 and are available to the community either through the solarsoft package FORWARD (Gibson et al. 2016) or by download from the Mauna Loa Solar Observatory website ${ }^{3}$. The circular polarization signal of these two Fe XIII lines is dominated by the Zeeman effect, while the Hanle effect dominates the linear polarization signal (Casini \& Judge 1999). While CoMP is mainly sensitive to the linear polarization, coronal circular polarization measurements at these Fe XIII lines will be provided by the Daniel K. Inouye Solar Telescope (DKIST; see Keil et al. 2011, and references therein), which first lights are planned for late 2019.

Theoretical (e.g., Judge et al. 2006; Rachmeler et al. 2013; Dalmasse et al. 2016) and observational (e.g., Bk-Stęślicka et al. 2013; Rachmeler et al. 2014; Gibson et al. 2017) studies have shown that Fe XIII, off-limb coronal polarimetry can distinguish between different 3D magnetic configurations such as, e.g. twisted flux ropes, sheared arcades, streamers, and pseudostreamers. However, coronal polarimetry alone is not enough to provide the $3 \mathrm{D}$ vector magnetic field in the full coronal volume. In addition to the limitations discussed Sect. 4.1.1, the Hanle effect associated with the Fe XIII lines operates in the saturated regime (Casini \& Judge 1999). In other words, the linear polarization signal is sensitive to the magnetic field direction but not its strength. Hence, while off-limb coronal polarimetry provides a unique type of information to constrain the vector magnetic field in the full coronal volume, it must be combined with other types of magnetic field measurements (e.g. photospheric or chromospheric) that are then integrated into 3D FFF and/or MHD models.

The data-optimized coronal field model $^{4}$ (DOCFM; Dalmasse et al. 2019) proposes such a solution by combining a parametrized FFF model with forward modeling of the coronal polarization signal in the Fe XIII lines. In this framework, the FFF model is parametrized through its electric currents $^{5}$, which can either be a surface-boundary parametriza-

\footnotetext{
3 https://mlso.hao.ucar.edu/mlso_data_calendar.php? calinst $=$ comp

4 https://www2 .hao.ucar.edu/hao-science/ data-optimized-coronal-field-model-docfm

5 The photospheric magnetic flux is not parametrized.
}

tion for FFF extrapolation methods, or a volume parametrization of the coronal electric currents for flux rope insertion methods (e.g., van Ballegooijen 2004; Titov et al. 2014, 2018). The parametrized FFF model is then optimized by minimizing the mean squared error between the polarization signal predicted for the FFF model and the real polarization signal (e.g. as observed by CoMP). The synthetic polarization signal associated with the FFF model is computed with the solarsoft FORWARD package (Gibson et al. 2016). In a recent study, Dalmasse et al. (2019) present a proof of concept using the flux rope insertion method of van Ballegooijen (2004) and CoMP-like data. They show that the DOCFM method opens new perspectives to retrieve the vector magnetic field in the coronal volume by combining off-limb coronal polarimetry with on-disk surface magnetograms and parametrized FFF models.

For an ideal application of the DOCFM approach, the region of interest must be simultaneously observed from two vantage points, i.e. from atop to obtain the on-disk surface magnetic field, and from the side to measure the corresponding off-limb coronal polarimetric signatures. Future coordinated observations of Solar Orbiter with DKIST - when Solar Orbiter is in quadrature with Earth - will thus provide unprecedented opportunities for simultaneous observations of on-disk surface magnetic fields and off-limb coronal polarimetry. Such measurements will constitute a unique set of observations to test new coronal magnetic field reconstruction techniques, infer the 3D coronal magnetic field of solar active regions, and better constrain the onset conditions of solar flares and CMEs.

\subsection{Synchronous synoptic maps of the corona}

The solar corona is a dynamics medium that can undergo global topological changes during the course of a solar rotation, the time typically required to build a Carrington map. It is therefore highly desirable to develop techniques that provide a more instantaneous mapping of the global atmosphere. This is particularly true in the context of Solar Orbiter that will provide detailed imaging from outside the ecliptic plane.

\subsubsection{Maps of the EUV corona}

By accumulating data (e.g. EUV intensity) from the central meridian in consecutive images and letting the Sun rotate under the observing S/C, it is possible to build Carrington maps of the corona, these coronal maps are similar to those described in Sect. 4.1.2 for the photosphere. By reprojecting from a different viewpoint, this allows simulating the aspect or radiance of the Sun from any point in the heliosphere (Auchère et al. 2005). Such maps are produced routinely from SDO data and made available online ${ }^{6}$.

Like photospheric magnetic field maps, data can be as old as one solar rotation period, while the structure of the corona can change in the matter of a few hours due to the emergence of new active regions or the occurrence of eruptions and CMEs. once again synchronic maps are therefore sought after that are for a given reference time instead of being for a given Carrington rotation. Of course it is always possible to reproject an image taken at some given time to Carrington coordinates, as made possible very easily in recent versions of JHelioviewer (Sect. 6.4.1), but then the resulting map covers only a small fraction (less than one half) of the solar sphere, while synchronous maps aim at covering the full sphere. Building these synchronous maps can

\footnotetext{
6 http://spaceweather.gmu.edu/projects/synop/AIASM. html
} 


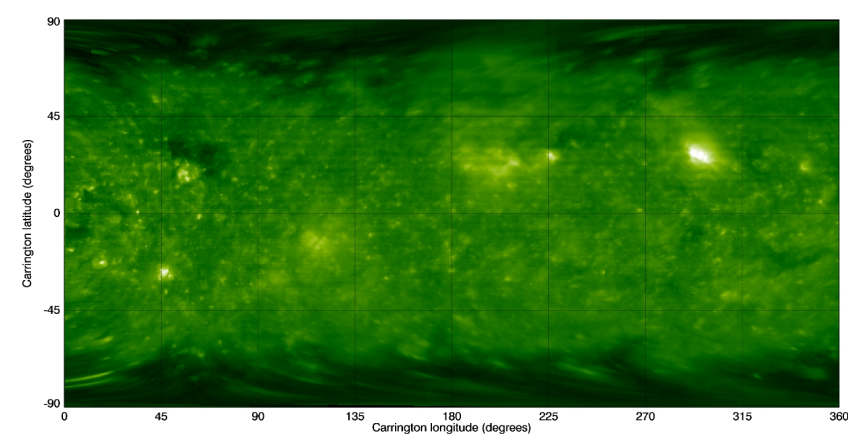

Fig. 6. Synchronous synoptic EUV map obtained for 2009-06-22 and displayed in Carrington coordinates. This map was constructed with $\mathrm{SOHO} / \mathrm{EIT}$ data and is made available by the MEDOC database.

be achieved in several ways, that can be combined for an optimal result. First, simultaneous data from different viewpoints can be combined, e.g. from instruments on different $\mathrm{S} / \mathrm{C}$, such as SECCHI/EUVI onboard STEREO A and B, and SOHO/EIT or SDO/AIA. Second, data from a large fraction of the on-disk corona (as observed from a given viewpoint) can be used instead of the central meridian only. Third, modelling can be used to simulate data in parts of the map that are not in any of the areas where observational data is available at the reference time. This can be as simple as taking into account the solar (differential) rotation from the closest point in time at which observational data was available (as in Auchère et al. 2005), but more complex models exist (as mentioned in Sect. 4.1.2 for the magnetic field).

A database obtained by producing synchronous EUV maps from SOHO/EIT in 1996-2010 is available as part of the MEDOC database ${ }^{7}$ (see Fig. 6). These maps are produced by taking $60^{\circ}$ on either side of the central meridian, and by computing differential rotation of older data in the rest of the solar sphere. Furthermore, for each pixel in the map, data from several EIT images are used, with weights related to the duration between the observation time (for these older data) and the reference time for the map. The same method has been applied to STEREO/SECCHI/EUVI data (from both STEREO S/C), and it is planned to make these maps available, together with new maps including SDO/AIA. Near-real time synchronous maps, for EUV radiance and for other quantities, can be useful to help planning Solar Orbiter observations. Also, Solar Orbiter/EUI will add an additional vantage point outside the ecliptic plane, providing EUV images of the polar regions.

\subsubsection{Maps of the white-light corona}

Coronagraphic WL synoptic maps are useful to obtain information on the large-scale structure of the coronal magnetic field through the comparison of the position of the quasi-stationary structures of the corona (as streamers, plumes, coronal holes), individuated in the maps, with the results of the coronal magnetic field extrapolations from photospheric data. Therefore, coronagraphic white-light maps can be used to optimize the coronal magnetic field extrapolations which are needed to establish the magnetic connectivity of the Solar Orbiter S/C with structures on the solar surface. This is an essential task to relate future RS and IS observations.

Different authors compared maps of the white-light corona at the source surface to PFSS extrapolations to understand the topological appearance and evolution of the coronal streamer

\footnotetext{
EUV-SYN dataset in http://idoc-medoc.ias.u-psud.fr/
}
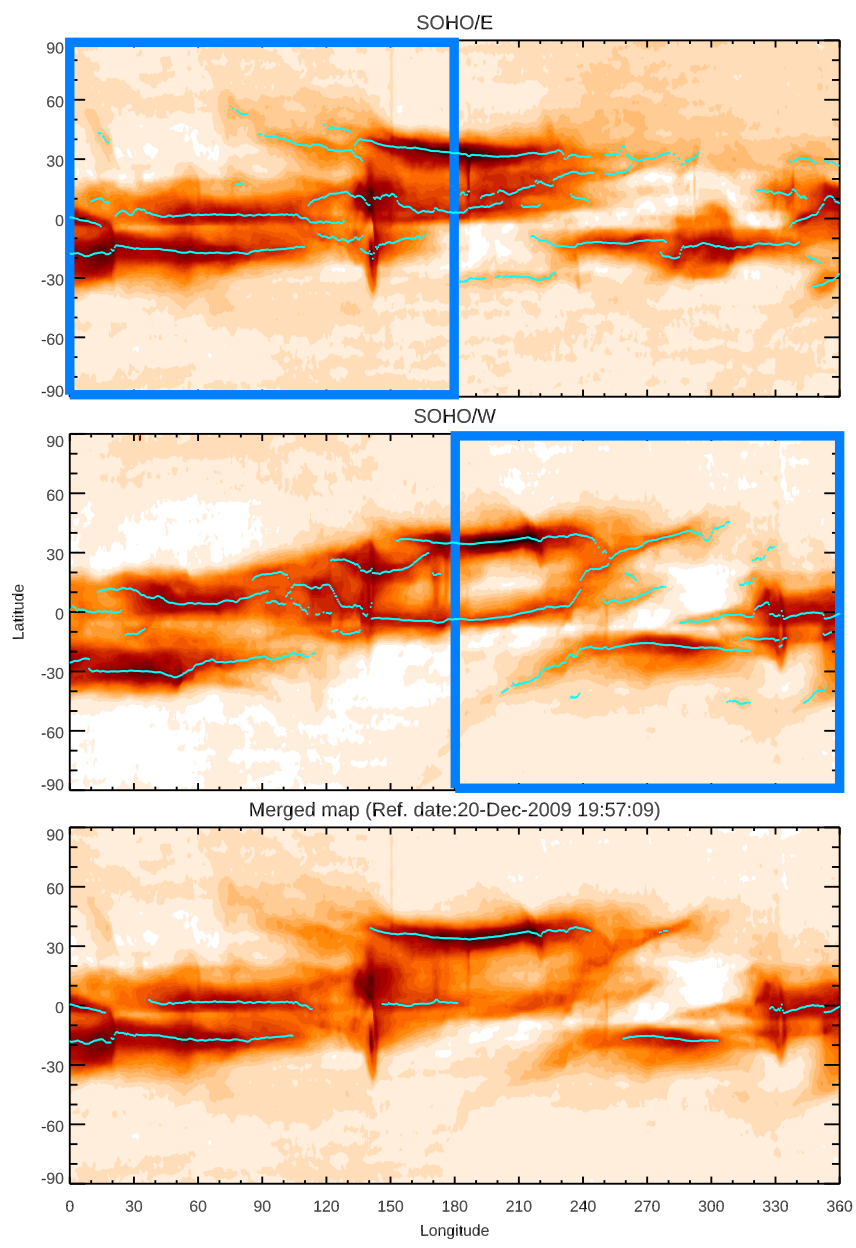

Fig. 7. East and west limb Carrington maps observed by the $\mathrm{SOHO} / \mathrm{LASCO}-\mathrm{C} 2$ coronagraphs, for the $\mathrm{CR} 2091$, at $2.5 R_{\odot}$ and the combined map obtained by their combinations (bottom panel), with the position of the streamers and pseudostreamers (cyan lines) overplotted. The blue box defines the part of the maps observed at the same time by the instrument.

belt that represents edgewise views of the coronal plasma surrounding the coronal current sheet, which is rotating with the Sun (Hoeksema et al. 1983; Koomen et al. 1998; Wang et al. 1997, 2000; Liewer et al. 2001). In particular, the comparison is done between the magnetic neutral line computed from coronal magnetic field extrapolations at $2.5 R_{\odot}$ and the position of the coronal streamer belt (Zhao et al. 2002; Saez et al. 2005). As for the magnetic and EUV maps, the assumption of no significant changes occurring of large-scale features is questionable during active times of the cycle. Traditional WL synoptic maps are built by using data taken at either the east or the west limb of the Sun. Obviously, by simply using observations from both limbs can halve the time needed to build a synoptic coronal map. An example of a synoptic map built over half of a solar rotation using both the east and Limb observations from LASCO during Carrington Rotation 2091 is shown in Fig. 7. Clearly, using observations from two or more vantage points can further reduce the time interval covered by a synoptic map.

Recently, combined quasi-synchronic white-light maps of the corona have been produced (Sasso et al. 2019) that provide the quasi-instantaneous positioning of streamers and pseudostreamers, two large-scale structures proposed as possible source regions of the slower solar wind. These maps are obtained from the combination of $2 \mathrm{D}$ coronagraphic Carrington maps 
from co-temporal data acquired by SOHO/LASCO-C2 and the two STEREO/SECCHI-COR1 instruments. Combining images from three coronagraphs observing the Sun from different viewing angles, we can produce Carrington maps covering the entire corona that provide the most accurate positioning of streamer structures at a particular time of interest, reducing the effect of temporal evolution. The synchronicity of the maps depends on the separation angles between the S/C. The combined Carrington maps, at the moment, are constructed by considering that the white-light measured by a coronagraph is scattered by electrons located in the plane of the sky. In a further step, we can carefully study line-of-sight effects on the derived longitudinal extent of the individual structures and the overlap of the various fields of views.

This work was part of the activities of the MADAWG and for this reason, to make the analysis more relevant to the Solar Orbiter mission, the maps were produced for a coronal configuration similar to the coronal structure expected for the early phase of the Solar Orbiter mission. In particular, the Carrington Rotation 2091 (CR, 2009 December 07-2010 January 03) was chosen as a representative time frame of the rising phase of solar cycle 24 . The relative positions of the three S/C (STEREO-SOHO separation angles were $\sim 65^{\circ}$ on 20 December 2009, 20:20 UT) were particularly favourable to scan the full corona in $\sim 5$ days (about $1 / 6$ th of a Carrington rotation), a time much shorter than a full rotation. This is an already significant improvement over the assumption underlying the typical usage of CR maps from a single vantage point, i.e. that the corona does not significantly change during a solar rotation.

Once the combined Carrington maps are created, it is possible to compare the position of the observed streamers in the maps with that of the neutral lines obtained from different magnetic field extrapolations, to evaluate the performances of the latter in the solar corona. Sasso et al. (2019) compared the observations with the results of four different PFSS extrapolations, showing that the location of coronal streamers can provide important indications to discriminate between different magnetic field extrapolations. The extrapolations are compared with the observations at the source surface, commonly placed at a height of $2.5 R_{\odot}$. A difficulty in this comparison arises from the fact that both helmet streamers and pseudostreamers contribute to the brightness of the $\mathrm{K}$ corona (hence, are both seen as bright structures in the coronagraphic white-light images), but only the former are associated with interplanetary sector boundaries and the heliospheric current sheet. The way to distinguish between a streamer or a pseudostreamer is via coronal magnetic field extrapolations. Other characteristic features of pseudostreamers, albeit more difficult to observe, are low-lying cusps and the presence of two underlying filament channels (Wang et al. 2007). However, even if it is not possible to distinguish between a streamer or a pseudostreamer without resorting to extrapolations, it is still possible to derive useful information on their reliability.

The work by Sasso et al. (2019) has also shown that such a technique can effectively discriminate between different magnetic field extrapolations, although some models predict positions of the current sheet that differ by just a few degrees in Carrington latitude and therefore are practically indistinguishable with this approach. Another limitation of this technique is that it is assumed that the observations are all taken from the plane of the ecliptic. In the context of MADAWG activities, this multi-viewpoint analysis can be re-done with other CR maps to cover, at least, one solar cycle; furthermore, other theoretical extrapolations should be included in the analysis, possibly including calculations not relying only on the PFSS approach.
After Solar Orbiter is launched, there will be several coronagraphs from space, i.e. Metis, ASPIICS on PROBA-3 to coordinate for joint observation campaigns. We have to take into account that for some instruments, like Metis that will observe also out of the ecliptic plane, there will be the need of other techniques, like tomography, to compare the data with the other coronagraphs (see Sect. 4.3.4).

\subsection{Inferring plasma parameters}

Different remote-sensing observations, like those recorded by Solar Orbiter, can be exploited to derive some basic physical parameters of the corona such as density, temperature and speeds. This section presents some of the techniques developed to achieve that with the upcoming Solar Orbiter data.

\subsubsection{Inverting Metis type-data}

A combination of well-established techniques and imaging inversion methods can be exploited to derive the main coronal physical parameters and their 2D distributions near the plane of the sky, on the basis of RS WL and UV observations. Coronagraphic WL images, such as those provided by the LASCO/SOHO instrument, are usually used to derive 2D electron density maps. The inversion technique of the polarized brightness, developed by van de Hulst (1950), allows to determine coronal electron density profiles $n_{\mathrm{e}}$ in the approximation of a spherical symmetry. The polarized brightness $(p B)$ is a function of the projected distance on the plane of the sky $\rho$, according to the following formula (Billings 1966; Hayes et al. 2001; van de Hulst 1950):

$p B(\rho)=C \int_{\rho}^{\infty} n_{\mathrm{e}}(r)[\mathcal{A}(r)-\mathcal{B}(r)] \frac{\rho^{2}}{r \sqrt{r^{2}-\rho^{2}}} \mathrm{~d} r$

where $r$ is the heliocentric distance, $C$ is a conversion factor, and $\mathcal{A}$ and $\mathcal{B}$ are geometric factors. The van de Hulst inversion technique can be applied with the assumption that the density profiles can be expressed by the polynomial form $n_{\mathrm{e}}(r)=\sum_{k}\left(\alpha_{k} r^{-k}\right)$, where $r$ is the heliocentric distance and $k$ is an integer varying between 1 and 4 (see e.g. Dolei et al. 2015; Hayes et al. 2001, for a more detailed description). A multivariate least-squares fit can be used to obtain the coefficients $\alpha_{k}$ that better reproduce the observed $p B$ radial profiles. These coefficients are then substituted into the expression of $n_{\mathrm{e}}(r)$ to derive the electron density radial profiles at all latitudes and thus obtain $2 \mathrm{D}$ density maps. Figure 8 shows, as an example, the electron density map in the altitude range $1.5-4.0 R_{\odot}$ derived from the inversion of a LASCO polarized brightness image.

The determination of the coronal density of free electrons provides a possibility to also derive their temperature $T_{\mathrm{e}}$, on which the ionisation balance of the coronal neutral hydrogen depends, as first described by Gibson et al. (1999). These authors developed their considerations for an ideal coronal plasma in hydrostatic equilibrium. This assumption can be reasonably applied to structures with outflow velocity values much lower than the sound speed (about $150 \mathrm{~km} \mathrm{~s}^{-1}$ for an isothermal corona at $10^{6} \mathrm{~K}$, see e.g. Priest 1987). Otherwise, the inference on electron temperature on the basis of the Gibson et al. (1999) approach gives only a first approximation estimate. Another way to derive the electron temperature in the solar corona is supplied by standard radial profiles reported in literature, which have been determined for specific coronal structures. The $T_{\mathrm{e}}$ values at all latitudes can be thus obtained by an interpolation of such profiles. 


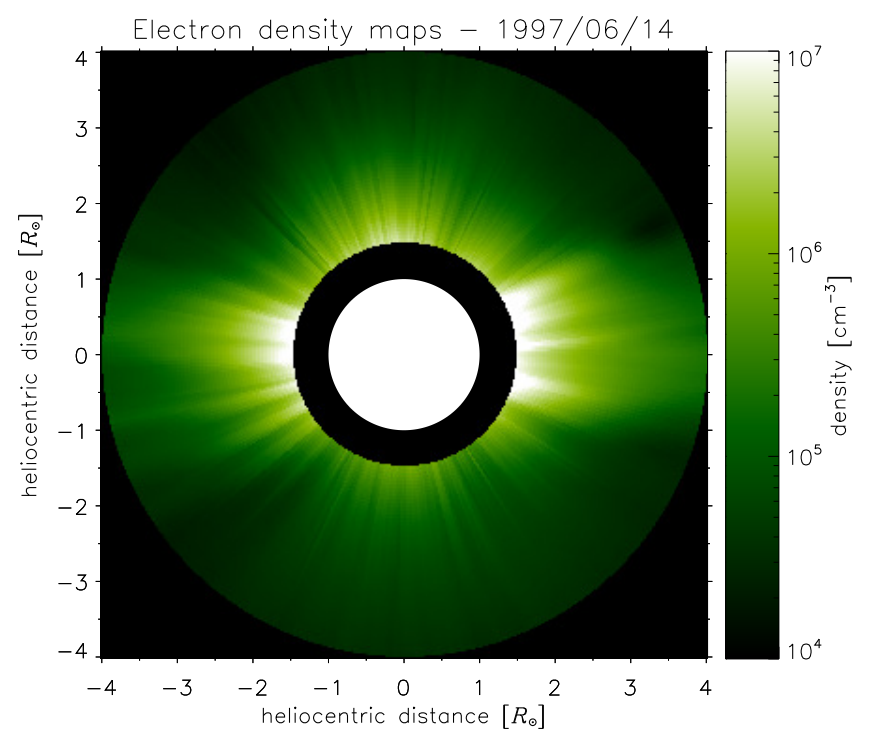

Fig. 8. Coronal electron density map derived by the van de Hulst inversion technique applied to a LASCO polarized brightness image on the 14th June 1997.

Investigation of solar wind outflow velocities is generally performed by the analysis of UV spectrometric observations. The UVCS/SOHO instrument has provided H I Ly $\alpha$ spectral line data over a longer time than a whole solar activity cycle (19962012), giving the possibility of studying coronal dynamics in different activity phases. The analysis of UVCS daily Ly $\alpha$ synoptic data, in combination with electron densities derived from the white-light RS observations, has allowed to derive H I outflow speed maps (see e.g. Dolei et al. 2018, 2019). One of the methods based on the synergy between UV and WL observations is the Doppler dimming technique (Hyder \& Lites 1970; Noci et al. 1987; Withbroe et al. 1982). It exploits the progressive UV intensity reduction of the coronal resonantly scattered component of the coronal H I Ly $\alpha$ line emission with increasing outflow velocities. The line emission depends on the physical quantities involved in the $\operatorname{Ly} \alpha$ resonant scattering process, such as, for instance, the coronal electron density and temperature, and the chromospheric Ly $\alpha$ radiation that excites the coronal $\mathrm{H} \mathrm{I}$ atoms. The Ly $\alpha$ intensity is also sensitive to the speed of the outflowing plasma from about $50-500 \mathrm{~km} \mathrm{~s}^{-1}$, that are the typical velocities for neutral hydrogen atoms in the inner corona. Following the approach of Withbroe et al. (1982) and Noci et al. (1987), the intensity of the resonantly scattered Ly $\alpha$ line can be also numerically computed by iteratively tuning the plasma speed value in order to reproduce the observed UV line intensity. The best match between computed and observed Ly $\alpha$ intensity provides an estimate of the solar wind H I outflow velocity. Figure 9 shows an example of 2D outflow velocity map, in the range of heliocentric distances between 1.5 and $4.0 R_{\odot}$, obtained via Doppler dimming technique (Dolei et al. 2018). The speed values radially increase with altitude up to about $150-200 \mathrm{~km} \mathrm{~s}^{-1}$ in the equatorial regions and $400 \mathrm{~km} \mathrm{~s}^{-1}$ in the polar regions. These values are in agreement with the expected latitudinal distribution of slow and fast solar wind components, corresponding to equatorial regions, and mid-latitude and polar regions, respectively. The methodology put in place throughout this project will be applied later to the data acquired by the Solar Orbiter's Metis instrument, giving an unprecedented daily picture of the coronal dynamics, see Antonucci et al. (2020) for a detailed description of diagnostic techniques for Metis data.

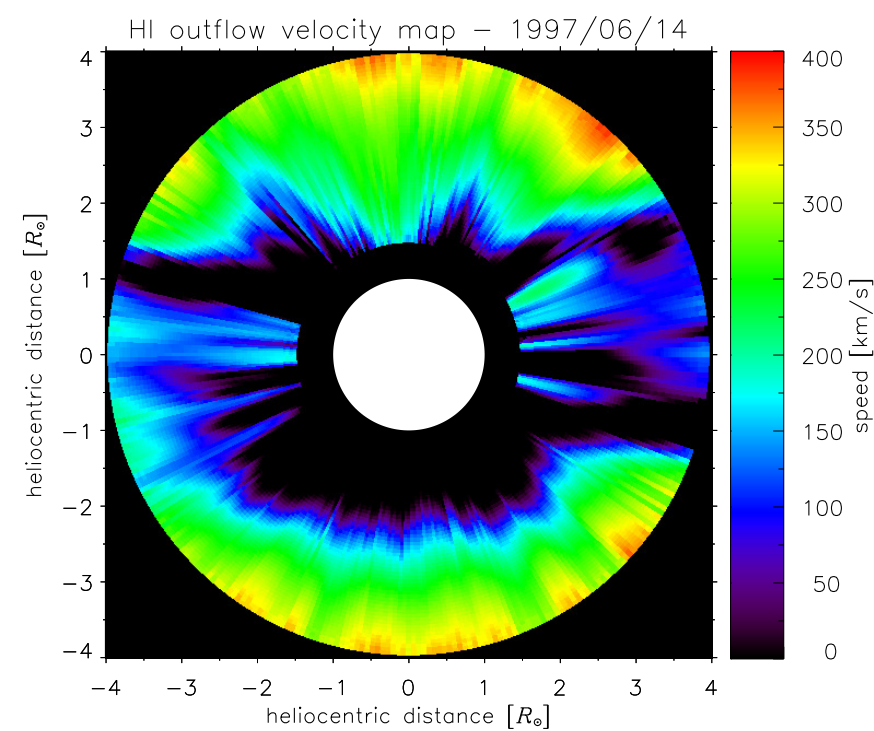

Fig. 9. Solar wind H I outflow velocity map on the 14th June 1997 obtained via Doppler dimming technique as described by Dolei et al. (2018) and exploiting both SoHO UVCS and LASCO observations.

The map reported in Fig. 9 has been obtained assuming a constant intensity value of the exciting chromospheric Ly $\alpha$ radiation, which has been derived from the Ly $\alpha$ irradiance measurements performed at 1 AU by the SOLSTICE/UARS instrument. As an alternative to the usual uniform-disk approximation, the distribution of the Ly $\alpha$ emission from the Sun should be determined. A representation of the daily chromospheric Ly $\alpha$ intensity distribution can be provided by means of a Carrington rotation map. It can be constructed using the solar disk observations acquired by the EIT/SOHO instrument in the $30.4 \mathrm{~nm}$ narrow bandpass filter (see Benevolenskaya et al. 2001) and then applying the correlation function between the full-disk intensities of the $\mathrm{H}$ I line at $121.6 \mathrm{~nm}$ and $\mathrm{He}$ II line at $30.4 \mathrm{~nm}$ established by Auchère (2005). The outflow speeds resulting in the cases of uniform and non-uniform disk intensity present significant differences. In particular, in the uniform-disk approximation the velocities are systematically higher than those obtained with the non-uniform chromospheric Ly $\alpha$ distribution in the Carrington map. The speed overestimate reaches its maximum value of about $50 \mathrm{~km} \mathrm{~s}^{-1}$ close to the Sun in the polar and mid-latitude coronal regions. This difference decreases at higher altitudes, where the portion of the solar surface visible from the corona tends to cover the full hemisphere. Both brighter and darker disk features can thus equally contribute to the illumination of the solar corona, and the non-uniform radiation condition progressively approaches the uniform-disk approximation. As for the exciting chromospheric Ly $\alpha$ line radiation, Metis investigation will be supported by the images of the entire solar disk in the He II line emission at $30.4 \mathrm{~nm}$ that will be provided almost simultaneously by the Full Sun Imager (FSI) of EUI aboard Solar Orbiter, after correcting for the contribution of the nearby Si XI line and applying the correlation function given by Auchère (2005).

\subsubsection{Inference on energy release and solar wind acceleration profile}

Once 2D maps of the electron density (hence mass density $\rho$ ) and outflow speed distribution have been derived on the plane of the sky (as shown by Dolei et al. 2018), these maps can be further analysed to provide very important information on the 
solar wind acceleration and coronal heating energy deposition processes. Following the analysis formerly performed by Holzer \& Leer (1980), Leer \& Holzer (1980), and Withbroe (1988), and most recently by Bemporad (2017), the first step corresponds to write the momentum equation for the solar wind dynamic, by considering a single fluid, stationary, and spherically symmetric solar wind flow, and by adding an unknown external force $f_{\text {ext }}$ responsible for the solar wind acceleration:

$\rho v \frac{\mathrm{d} v}{\mathrm{~d} r}=-\frac{\mathrm{d} P_{\mathrm{gas}}}{\mathrm{d} r}-\frac{G M_{\odot} \rho}{r^{2}}+f_{\mathrm{ext}}$

where $v(r)$ is the radial wind speed, $\rho(r)$ is the plasma mass density, and $P_{\text {gas }}(r)=2 n_{\mathrm{e}} k_{\mathrm{B}} T(r)=\left[\rho(r) / \mu m_{\mathrm{H}}\right] k_{\mathrm{B}} T(r)$ is the gas pressure given by the perfect gas law. In the above equation, terms $\rho(r)$ and $v(r)$ are provided directly with the analysis of coronagraphic data, and the $2 \mathrm{D} v(r)$ distribution can be converted in a radial gradient image showing the plane-of-sky distribution of $\mathrm{d} v / \mathrm{d} r$. Hence, the only missing term is the gas pressure gradient

$$
\frac{\mathrm{d} P_{\text {gas }}}{\mathrm{d} r}=\frac{k_{\mathrm{B}}}{\mu m_{\mathrm{H}}}\left[\frac{\mathrm{d} \rho}{\mathrm{d} r} T(r)+\rho(r) \frac{\mathrm{d} T}{\mathrm{~d} r}\right]
$$

and again in the above expression the $2 \mathrm{D} \rho(r)$ distribution from the analysis of coronagraphic data can be converted in a radial gradient image showing the plane-of-sky distribution of $\mathrm{d} \rho / \mathrm{d} r$. In the end, once a radial temperature profile $T(r)$ is assumed for instance from the literature, this can be used to derived a temperature radial gradient profile $\mathrm{d} T / \mathrm{d} r$, and in the end to measure the $2 \mathrm{D}$ distribution of the unknown external force $f_{\text {ext }}$ responsible for the solar wind acceleration. This information is important to determine where fast and slow wind are mostly accelerated by the additional force $f_{\text {ext }}$, and to suggest possible physical explanations for it.

Moreover, the derived plane-of-sky distribution of mass density and outflow speed can be combined to derive further information on the solar corona. First, 2D images of the solar wind mass flux distribution $F_{\mathrm{w}}$ can be derived as $F_{\mathrm{w}}=v \rho r^{2} f_{\mathrm{w}}$, with $f_{\mathrm{w}} \geq 1$ unknown distribution of the flux tube expansion factor, and $A(r)=f_{\mathrm{w}}(r) r^{2}$ cross sectional area of the flux tube. For instance, by simply assuming $f_{\mathrm{w}}=1$ the 2D distribution of $F_{\mathrm{w}}$ will provide information on regions where super-radial expansion is going on or not (depending on whether $F_{\mathrm{w}}$ is constant or not with altitude). Alternatively, different functional forms for $A(r)$ can be tested in different regions of the corona to have $F_{\text {w }}$ conservation in the end. Potentiality of the above techniques for solar wind studies was recently shown by Bemporad (2017) based on the analysis of combined SOHO/UVCS and LASCO observations. Connection with IS data will also provide further constraints for instance during future Solar Orbiter-PSP quadratures, as previously tested during the $\mathrm{SOHO}-$ Ulysses quadrature campaigns (e.g. Suess et al. 2000; Bemporad et al. 2003).

Also, the $\rho(r)$ and $v(r)$ images will allow (under some assumptions) to measure the 2D distribution of coronal expansion times (see e.g. Withbroe et al. 1982), to be compared with possible values for other important time scales, such as the hydrogen-proton charge exchange time and the hydrogen (collisional and radiative) ionization time (see e.g. Spadaro et al. 2017), thus allowing to identify inner and outer coronal regions where neutral hydrogen and coronal protons can be considered coupled or decoupled, respectively. This is very important to discriminate coronal regions where the measured outflow speeds (relative to neutral hydrogen atoms) can be considered as representative of solar wind proton speeds. This will also provide information on the proton freeze-in distance in the solar corona (see e.g. Boe et al. 2018), thus allowing a better link between RS and IS observations. Last but not least, the 2D distribution of density and outflow speed can be combined to extend previous techniques that have been proposed (e.g. Habbal \& Esser 1994) to routinely estimate an upper limit to the helium coronal abundance.

Hence, the availability of 2D electron density and outflow speed maps will allow the development of many other tools allowing also to study solar wind acceleration and coronal heating problems, and to determine many other physical properties of coronal plasma.

\subsubsection{Differential emission measure}

As the coronal emission is optically thin, any line of sight crosses structures with inhomogeneous temperatures and densities. The temperature differences are the consequence of heating, conductive losses, magnetic flux emergence, radiative losses, and, of particular interest here, energy losses associated with the escaping solar wind. Images taken at a specific wavelength (typically in the EUV), corresponding to an emission line that results from the de-excitation of an ion, can provide a picture of the solar corona at a certain temperature, but the multi-temperature nature of the plasma in the volume determined by the line-of-sight and the pixel size can only be captured using a larger number of lines radiances. Thanks to the properties of coronal line emission, the line radiances can be expressed as a temperature integral of a "contribution function", specific to each line and related to the atomic physics of line emission, multiplied by the "Differential Emission Measure" (DEM), which can be expressed as $\operatorname{DEM}(T)=n_{\mathrm{e}} n_{\mathrm{H}} \mathrm{d} h / \mathrm{d} T$, where $\mathrm{d} h$ is the length on the line-ofsight where the plasma temperature is between $T$ and $T+\mathrm{d} T$. The DEM represents then quantitatively the amount of plasma (as its square density) as a function of temperature.

As the DEM depends on the physical parameters of the plasma only, and not on the atomic physics of each line, it can be inverted from the observed radiances in different spectroscopic lines (observed by spectrometers such as SOHO/SUMER and Hinode/EIS) or in wavelength bands (observed by typically narrow-band imagers, such as SDO/AIA). This is a mathematically difficult problem, but many methods have been developed to perform this inversion (see Sect. 7 of Del Zanna \& Mason 2018, and references therein). One way to overcome the intrinsic underdetermination of the problem is to assume that $\operatorname{DEM}(\log T)$ belongs to a given class of functions, like a simple or multi-Gaussian (Aschwanden \& Boerner 2011; Del Zanna 2013) function of $\log T$, the parameters of the function being determined via an optimization scheme, often based on chi-square minimization. The accuracy of this type of approach using SDO/AIA data is tested in Guennou et al. (2012a,b). Many strategies have been explored to find the most probable solution through the vast parameter space while minimizing the number of ad-hoc assumptions, e.g. Markov-chain Monte Carlo algorithms (Kashyap \& Drake 1998), regularization in L2 (Hannah \& Kontar 2012), sparse inversion in L2 (Warren et al. 2017) or L1 (Cheung et al. 2015). DEM inversion is still an active field of research, and there is no single method adopted by the community as providing robust and undisputed results. The choice of method generally depends on the application and results from a compromise between computing time, accuracy and robustness.

A database of DEM inversions obtained by assuming a Gaussian DEM on all SDO/AIA data at a cadence of 30min is available as part of the MEDOC database ${ }^{8}$ (see Fig. 10). During

\footnotetext{
8 Gaia-DEM dataset in http: //idoc-medoc.ias.u-psud.fr/
} 

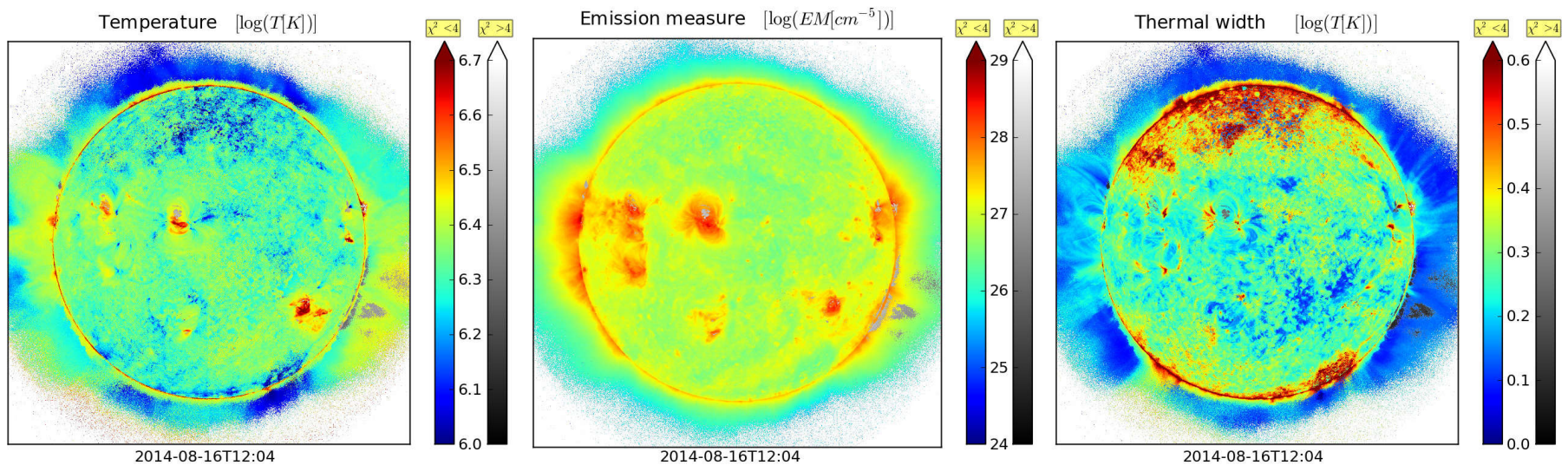

Fig. 10. Maps of the three Gaussian DEM parameters (temperature, emission measure, thermal width) included in the Gaia-DEM database, for 2014-08-16T12:04. These maps were produced by using EUV images from the SDO AIA instrument.

the Solar Orbiter era, it is planned to continue maintaining this database and completing it with new DEM inversion results. Possible evolutions include the use of functional DEMs with a high-temperature tail, that would be more adapted to active regions, or the use of other instruments such as the NOAA operational instrument GOES-16/SUVI. On Solar Orbiter, the SPICE spectrometer will have the capability to provide data for DEM inversions. The EUI $17.4 \mathrm{mn}$ channel could also help constrain the DEM, as well as STIX in the high-temperature range (see e.g Schmelz et al. 2009, for DEM inversions from Hinode/XRT combined with RHESSI). However, DEM inversions involving SPICE cannot be computed systematically, as this depends on the choice of wavelength windows in the study used for the SPICE observation. The design of SPICE studies will have to take into account the adequacy of this choice to be able to perform DEM inversions.

\subsubsection{Tomographic reconstruction}

Tomography is another inversion method that has the potential to provide estimates of the 3D distribution, not only of the electron density, but also of the temperature and emission measure of the corona (Davila 1994). It can be applied in the inversion of EUV (Vásquez et al. 2009, 2011; Barbey et al. 2008, 2013) and WL observations (Frazin \& Kamalabadi 2005a,b; Kramar et al. 2009; Frazin et al. 2010), both of them readily available in the Solar Orbiter mission. The information on the 3D structure of the corona is provided by the rotation of the Sun (rotational tomography), under the assumption that the corona remains static during the period of interest (at least half a solar rotation is generally required for a single viewpoint observation). This assumption can be somewhat relaxed if the temporal evolution is assumed to occur mostly in specific structures (Barbey et al. 2008), or when observations from multiple vantage points are available, as is the case since the launch of the STEREO mission (Frazin \& Kamalabadi 2005a,b; Kramar et al. 2009). The amount of solar rotation required to capture the necessary information for the tomographic reconstruction becomes shorter as the angular distance (and hence the coronal coverage) of the two S/C increases. Nominally, a single week is required when the two $\mathrm{S} / \mathrm{C}$ are in quadrature. This, however, has not been demonstrated in practice. For example, Vásquez et al. (2011) required 24 days for their EUV inversions with the STEREO S/C $\sim 48^{\circ}$ apart.

It should be obvious that a static corona over a full rotation is a rather strong assumption, even during solar minima. Impulsive activity tends to create artefacts in the tomographic reconstructions and CMEs, particularly fast ones, can remove mass over large swaths of the coronal volume, complicating greatly the reconstructions. Furthermore, the EUV and white light lines of sight may contain contributions from structures over wide longitudinal ranges since both emissions are optically thin and these contribution can be different for the various viewpoints. Efforts to deploy reconstructions that account for the varying corona are under way (e.g. Vibert et al. 2016), but require additional constraints that the currently available 2 or 3 lines of sights, which all lie in the ecliptic plane cannot easily provide.

Solar Orbiter is poised to make significant impact in tomographic reconstructions thanks to two unique aspects of the mission: namely, the off-ecliptic viewing geometry and the elliptical orbit. The former breaks the degeneracy of the ecliptic imaging from STEREO, SDO, and SOHO, delivering strong constraints on the longitudinal extent of streamers and the roots of polar plumes. The elliptical orbit, on the other hand, results in a varying distance between observer and coronal structures, which affects the Thomson scattering behaviour, discussed in more detail in Sect. 2.5.2 in Howard et al. (2020). Although the Thomson scattering considerations are more relevant for WL imaging at large elongations, subtle differences in the observed brightness will provide some information on the location of a given structure seen from different viewpoints (say, from STEREO/COR, $\mathrm{SOHO} / \mathrm{LASCO}$ and Solar Orbiter/Metis). Tomography in this context is discussed in Howard et al. (2020) and Vourlidas et al. (2016).

Overall, we expect that EUV tomography will benefit first (and mostly) from the Solar Orbiter observations because its an easier inversion problem and can make use of stereoscopy for the small angular separations (up to $\sim 10^{\circ}$ ) in the early science phase of the mission. White light reconstructions of the extended corona will have to wait until Solar Orbiter reaches higher elevations, probably $20^{\circ}$ or more, to provide sufficiently different viewpoints than the telescopes on the ecliptic. Close coordination with these assets will be necessary to optimize the reconstructions and derive the 3D distributions of density, temperature and emission measure.

\section{Magnetic connectivity}

The previous sections of the paper have discussed strategic developmenets aiming at improving our understanding of coronal plasma parameters. To establish physical connection between RS observations of the Sun and IS measurements made along its orbit implies estimating systematically (and automatically) the 
magnetic connectivity between the Sun and the S/C at all times, both for past events and as a forecast (with at least a few days lead time). The outcome of this modeling effort will serve both Solar Orbiter science operators with a tool to aid observation planning, and the scientific community with an invaluable new tool for data analysis. A number of difficulties arise, the major ones being the need to coordinate many different datasets and numerical models, the large number of sources of uncertainty in the data, the intrinsic biases of the models, and the need to couple different physical domains.

As a first step and in order to address the problem of magnetic connectivity, the MADAWG proposed the development of a new modular modeling framework/pipeline that brings together different models from the community and that can be set up to work equally well for forecasting connectivity and for post-event analysis. This "connectivity tool" relies on a back-end pipeline that works continuously and in real-time, and on web-based front-ends. The tool relies on simple and well established models for robustness and ease of analysis during operations. It performs multiple simultaneous estimates of connectivity based on different combinations of models and datasets rather than choosing only one model/data combination. This is a way to assess the quality and uncertainty of the connectivity estimates. The computational work related to running different models/datasets will be distributed by the model developers, with only a reduced and standardised sets of parameters being transferred regularly between different institutions to the tool. Nevertheless, one full branch of models and datasets are run locally in order to guarantee the capability to produce daily end-to-end connectivity estimations (and to prevent against eventual failures in communications).

\subsection{Connectivity tool layout}

Backtracing of magnetic field lines is performed from a given $\mathrm{S} / \mathrm{C}$ position down to the surface of the Sun following a pipeline composed of three main modules (initiation, field-line tracing, foot-point positions). The initiation step sets up the pipeline to operate in post-event analysis or in forecast mode according to the date or $\mathrm{S} / \mathrm{C}$ position chosen, and performs the corresponding checkups (e.g., verify raw and pre-computed data availability). The field line tracing module separates into two substeps, corresponding respectively to the heliospheric and to the coronal part of the domain: in its simplest configuration suitable for operations, the tool traces a Parker spiral (idealized or extracted from a numerical model) from the $\mathrm{S} / \mathrm{C}$ position down to the pre-defined coronal boundary, and then uses a method of coronal field reconstruction to trace the field lines down to the surface of the Sun.

Several tracing methods are available at both stages according to the operation mode. Differences between operation in forecast or in science mode consist essentially in using archived or forecast magnetograms, and on using in situ measurements of solar wind speed to constrain the shape of the Parker spiral. Finally, the last module traces the magnetic footpoints at the surface of the sun taking into account the estimated Sun-S/C propagation delays for different types of phenomena and perform estimations of connectivity uncertainty compared to the RS instruments fields of view.

Observation and model data storage is ensured by the PI institution of each respective dataset, and only a minimal subset of parameters is requested and downloaded by the server running

9 Accessible at http://connect-tool.irap.omp.eu/
1. Initiation

Science mode or Forecast mode
(according to choice of date or $\mathrm{s} / \mathrm{c}$ position)

2. Field-line tracing

\begin{tabular}{|c|}
\hline $\begin{array}{l}\text { 2.i) Heliospheric model (or "Parker spiral”) } \\
\text { use in-situ wind speed use estimated wind speed } \\
\text { 2.ii) Coronal field reconstruction } \\
\text { use archived magnetograms use forecast magnetograms }\end{array}$ \\
\hline 3. Find connectivity regions at the solar surface \\
\hline $\begin{array}{l}\text { Take into account propagation delays (wind, particles) } \\
\text { Plot maps at plasma release times and at target time } \\
\text { Determine connectivity probability distributions } \\
\text { Compare uncertainties to RS instrument FOV }\end{array}$ \\
\hline
\end{tabular}

Fig. 11. Layout of the connectivity tool pipeline showing its three main modules, and the subdivision of the fieldline tracing module into two steps (corresponding to the heliosheric and coronal domains). The pipeline has two modes of operations - science/post-event analysis and forecast modes - that share the same general scheme but that require using different data sources and models (highlighted respectively in blue and green).

the connectivity tool to initiate each of its modules in order to minimise the required daily data transfer rate. Furthermore, the host server is able to perform autonomously and locally full endto-end connectivity estimates based on at least one combination of models and datasets in order to overcome possible datalink failures (see Fig. 11).

\subsection{Connectivity tool for operations}

We now describe how the Connectivity Tool will be exploited to help Solar Orbiter operations before and after acquisition of the data.

\subsubsection{Operational context and requirements}

Solar Orbiter's operational constraints result mainly from the off-line commanding concept of the mission, the restricted time during which RS instruments can operate, and the severely limited telemetry downlink resulting in significant data latency. The highly elliptical orbits around the Sun cause a very strong variation in data downlink rates, up to a factor 23 between the best downlink regime (close to Earth) and the worst one when Solar Orbiter is observing the far side of the Sun (at a distance up to 2 AU from Earth). This results in severe TM restrictions for the mission and has led to a mission baseline in which the full payload can only be operated during a limited time along the orbit. $\mathrm{RS}$ observations are restricted to so-called RS windows, typically three 10-day periods per orbit, to be placed at the scientifically most interesting opportunities. Even so, science data cannot be downlinked immediately after acquisition and will need to be stored onboard and queue for downlink. Due to the resulting latency in science data, which may take up to six months, a solution is needed to safeguard the visibility on payload performance and safety. Low-Latency Data (LLD) is a limited set of (lower quality) science data that has been designed to fit in the daily TM stream and will help the Science Operations team to evaluate instrument health, data quality, and recent solar 


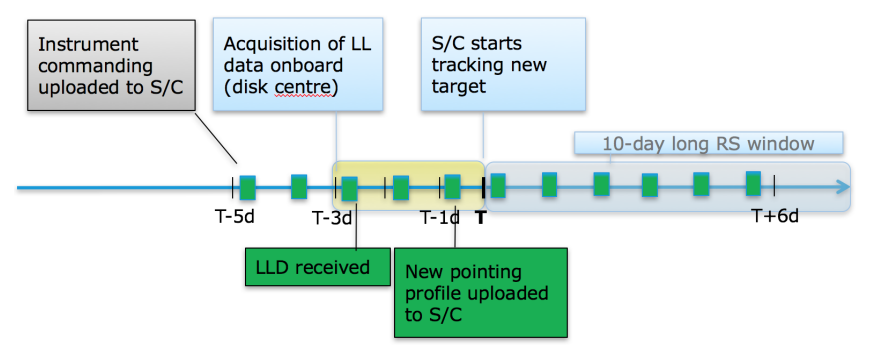

Fig. 12. Schematic of the operational timeline along which decisions have to be taken in order to point the S/C at an appropriate target at time $T$. The 10 day long RS window is shown as a blue shading starting at time $T$, the yellow shading depicts the pre-RSW period during which RS instruments will be preparing for the science phase and collecting LL data. Daily passes are shown in green. As shown in grey, typically all instrument commanding will be onboard before the first instrument is switched on. The LLD acquired during the pre-RSW period will provide context to choose $\mathrm{S} / \mathrm{C}$ pointing which can be adjusted with less lead time. Green boxes provide information on when ground receives LLD or send commands, blue boxes show what happens onboard. This process is repeated every day throughout the preparation days and actual RS window.

activity. For more details, see Sanchez et al. (in prep.) and Zouganelis et al. (2020).

On the uplink side, instrument commanding for a full week of operations (also referred to as short-term planning) will be uploaded at once, typically a few days before the first command is executed on-board. So while instrument commanding cannot be changed daily (with the exception of a few calibrationrelated parameters, see Sanchez et al., in prep.). The LLD may be used to evaluate suitability of the target captured in the highresolution FOVs and if necessary re-adjust the $\mathrm{S} / \mathrm{C}$ pointing. The $\mathrm{S} / \mathrm{C}$ and all instruments' line of sights with it can be re-pointed on the solar disk on a daily basis, as part of the VSTP. Nevertheless there will be 3Days between acquisition of the LLD and the actual occurrence of the re-pointing. Figure 12 summarizes the operations timeline. In order to start high-resolution observations of an appropriate target on the first day of the 10-day window, labelled as time $T$ in the figure, the RS instruments will be switched on a few days earlier (a) to prepare the instruments for qualitative science observations (e.g. by performing a minimal calibration campaign) and (b) to collect LLD that will help choosing the right target on the Sun. At least 3 days of pre-RSW operations (highlighted in yellow in Fig. 12) will be needed to point the $\mathrm{S} / \mathrm{C}$ to the target in time. The first full-disk LLD will be collected at time $T-3 \mathrm{~d}$ and downlinked during the following pass. The latest pass at which the new pointing profile needs to be uploaded takes place in the $24 \mathrm{~h}$ before time $T$. This leaves roughly $36 \mathrm{~h}$ to (1) analyse the LLD, (2) choose a target on the Sun based on modelled connectivity or the chance for particular events, (3) calculate the appropriate $\mathrm{S} / \mathrm{C}$ pointing profile, and (4) prepare the commanding to be uploaded during the last pass before time $T$. In practice this means that the time available to decide on the adjusted pointing is about $12 \mathrm{~h}$. It is worth noting that each day, new LLD can be downlinked for analysis and based on this new context information, one may decide to adjust the pointing. Although this process can be repeated every day throughout the RS window, the adjusted pointing profile will only take effect 3 days later than the time the LLD were acquired.

The LLD for each instrument have been defined to best serve evaluation of instrument health, data quality and S/C pointing accuracy. In addition, LLD can be used to shed a light on the current solar activity, including the presence of potential targets, to provide context for the connectivity models, and to decide which data to prioritize or select for downlink ${ }^{10}$. As it stands, EUI LLD contain full disk, highly compressed images in $304 \AA$ and $171 \AA$ at $15-30$ min cadence plus one higher quality dataset per day. PHI LLD consists of one full disk magnetogram and a continuum intensity image at medium resolution. Metis LLD provides one set of white-light and ultraviolet images of the corona and light curves in 8 coronal sectors. Solar OrbiterHI LDD provides a low resolution J-map. STIX LLD is a series of lightcurves in several X-ray energy bands + rough flare locations. The IS instruments EPD, MAG, SWA, and RPW provide continuous low-cadence measurements of normal mode: energetic particles at all energies, magnetic field vectors, proton moments, strahl electron distribution, $1 \mathrm{MHz}$ radio measurements. Things we cannot do include (1) changing the available pointing intervals, since slew opportunities are defined at the long-term planning level, such that instruments can plan around them (2) changing the roll angle, prime boresight and instrument planning.

The models must be capable of giving the most likely connection between Solar Orbiter and a future target of interest on the solar disk (typically 3 days from the current time and onwards). The connectivity tool must run with synoptic or synchronic magnetogram data from other observatories complemented when relevant/possible with the Solar Orbiter LLD. This data will transit via the Solar Orbiter Ground Segment (Sanchez et al., in prep.) where it will be fetched and exploited by the connectivity tool. In particular we should be able to ingest additional input if available (e.g. far-side magnetograms from Earth-bound assets). The tool should predict the connection between plasma/energetic particles passing at Solar Orbiter and the solar sources several days in advance so that we can compare directly to the most recent images of the Sun (from Solar Orbiter LLD or other relevant data sources). This connection must be established by accounting for propagation times of CMEs, solar wind plasma and energetic particles. An additional requirement for operational support is good stability of the tools.

\subsubsection{Methodology to forecast connectivity before data acquisition}

Forecasting sun-to-S/C connectivity requires combining fast modeling methods with magnetogram forecasting techniques, to devise ways of constraining the associated uncertainties, and to provide diagnostics for validation. As already stated, the connectivity tool in forecast mode models the corona with the PFSS model using ADAPT maps as boundary conditions (or any form of time-evolved magnetograms) and extends the coronal magnetic field to the probe using a Parker spiral computed using assumed rather than measured wind speed (see Fig. 13). As we discuss in Sect. 5.4, the sources of uncertainty are numerous and of different types. These include, on one hand, errors in surface magnetic field measurements, errors in the extrapolation methods, biases related to physical assumptions related to the corona and to the state of the solar wind, and on the other hand, uncertainties due to the occurrence of bifurcations in neighbouring magnetic paths (especially close to the HCS or across boundaries between magnetic sectors). The former can typically be assigned a systematic error, while the latter can produce sudden

${ }^{10}$ Only instruments with internal memories, like EUI, PHI and STIX, can manage data priorities before sending to the S/C memory. Selective downlink from the S/C shared memory is reserved for RPW and EPD only. See Sanchez et al. (in prep.) for more details. 

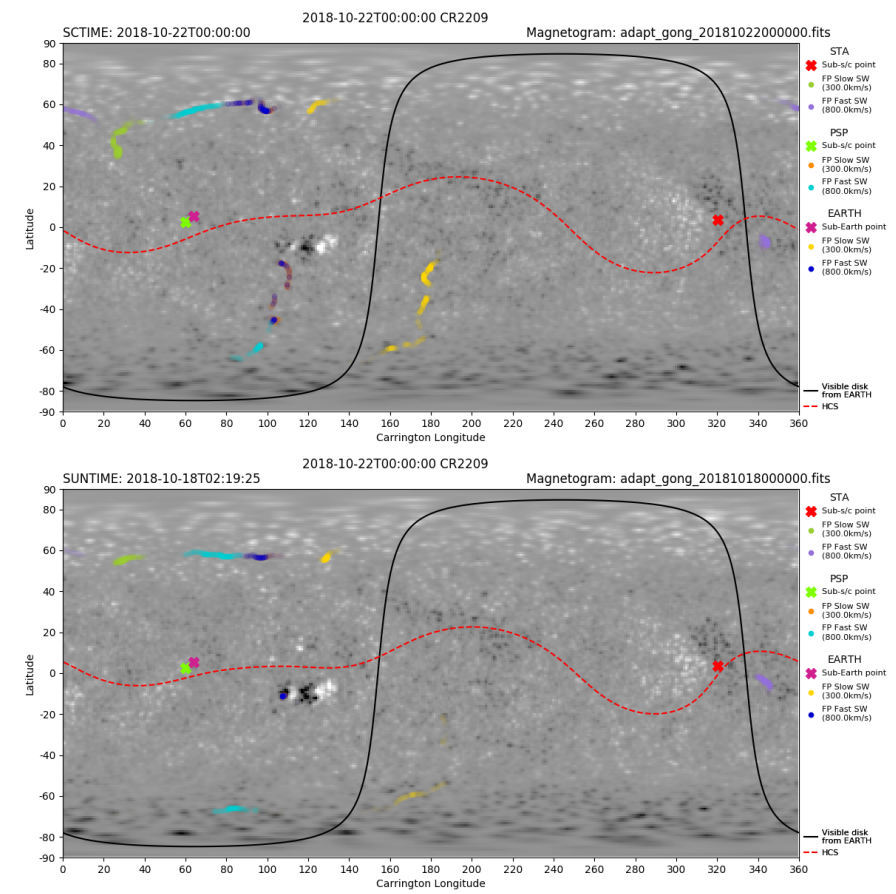

Fig. 13. Examples of connectivity maps forecasted 2 days in advance by the connectivity tool using ADAPT/GONG magnetic field maps a simple heuristic estimate for the speed of the wind stream reaching the S/C (PSP on this example). Zones of high connectivity probability are shown for PSP, Earth and STEREO-A, for assumed slow and fast solar winds. Top panel: instantaneous magnetic connectivity at the time considered, bottom panel: connectivity footpoints at the time of the expected solar wind plasma release, highlighting the subtle differences between both. These maps clearly show how proximity to strong topological features such as large equatorial streamers and the HCS can affect the estimations of connectivity (sometimes more severely during solar minima), resulting in this case in wide latitudinal separations (cf. points of the same colour both on the northern and southern hemispheres). Solar Orbiter LLD data will be fundamental to help establishing the most likely source of plasma in these situations.

jumps in connectivity, and even multiple regions of connectivity likelihood that can be disconnected and placed at large distances over the surface of the Sun. In an operational context, data from SWA/PAS-LLD and SWA/MAG-LLD will provide the plasma speed measured IS from which we can establish the likely source of the plasma at the Sun when the plasma was released several days before. We can then use MAG-LLD to figure out in which magnetic sector Solar Orbiter is located and tackle the most unpredictable of the two types of sources of uncertainties described above. This provides valuable information to evaluate the accuracy and to validate the magnetic connectivity models up to the time $T-1 \mathrm{~d}$ of uploading commands to Solar Orbiter for camera repointing. In this approach we will use the in situ data to continually evaluate different output of the coronal models as we approach the RSW.

Up to uploading the command, we then have two options for the boundary conditions of the PFSS model. The first one is to use magnetograms that are built from magnetic field data taken up to the last possible time before commands have to be uploaded to the $\mathrm{S} / \mathrm{C}$ and consider that they are the best available boundary conditions to derive the global coronal topology. Ideally this step would incorporate PHI-LLD data that will provide surface magnetograms outside the Sun-Earth line. A second and even better approach is to take the most recent magnetograms and apply a flux transport model to forecast the distribution of magnetic fields several days in advance. The ADAPT modelling framework provides such maps as discussed in Sect. 4.1.2. For each model result we can then compare the derived positions of coronal holes and active regions to EUI-LLD as well as streamers imaged by from METIS-LLD which will provide a first evaluation of the quality of the forecasts and help us in our choice of target pointing.

As support to the interplanetary part of the magnetic field modelling, solar wind models that run from the Sun to Solar Orbiter can also provide additional information on the solar wind speed that will be encountered by Solar Orbiter. These include global solar wind models, wind propagation models and empirical relations based on the geometry of solar magnetic flux tubes. Lacking information on solar wind speed that Solar Orbiter will measure three days later, we have to assume extreme values of the background solar wind speed (e.g., $300-800 \mathrm{~km} \mathrm{~s}^{-1}$ ) in order to map the uncertainty in the shape of the Parker spiral.

However, in support to the interplanetary part of the magnetic field modelling, solar wind models that run from the Sun to Solar Orbiter can also provide additional information on the solar wind speed that will be encountered by Solar Orbiter. These include global MHD solar wind models, wind propagation models (such as EUHFORIA) and empirical relations based on the geometry of solar magnetic flux tubes. Importantly, new strategies that aim at forecasting the solar wind conditions at any point in the interplanetary space have been seeing significant developments over recent times. Among these, the SWiFT-FORECAST modeling pipeline takes full advantage of multi-1D wind models (such as MULTI-VP) combined with time-evolved synchronic magnetograms (such as those of ADAPT) to provide continuous realtime nowcasts and few-day forecasts of the solar wind conditions at any $\mathrm{S} / \mathrm{C}$ position. The Heliopropa service ${ }^{11}$ propagates measured IS solar wind data from given orbital position to another point in the interplanetary medium, and can provide very accurate solar wind forecasts if the source and target orbital positions are favourable. Full 3D models of the heliosphere (such as EUHFORIA) will also provide an important contribution, especially regarding the state of the solar wind in the outer atmosphere and during strong transient events.

In its current configuration, the EUHFORIA model (Pomoell $\&$ Poedts 2018) solves the 3D MHD equations but the coronal part uses the Wang-Sheeley-Arge (WSA) emperical law. EUHFORIA (Pomoell \& Poedts 2018) is a newly developed model to forecast the space weather conditions on the inner heliosphere. The code uses magnetograms to extrapolate semi-empirically the inner coronal field from the solar surface up to boundary conditions at the so-called source surface typically set at $R=2.5 R_{\odot}$, and then extend the magnetic field to the model's lower boundary at $R=0.1 \mathrm{AU}$. The current version of the model uses the WSA emperical law. From there onwards, a full 3D MHD simulation models the evolution of the solar wind. Different from ENLIL, EUHFORIA allows to insert CMEs not as plasma blobs, but as genuine magnetic structures.

In its most advanced setup, the Wang-Sheeley-Arge (WSA) model (Arge et al. 2004) uses a set of twelve ADAPT maps to generate an ensemble of daily (or higher cadence) predictions of Solar Orbiter's magnetic connectivity to the Sun. WSA can forecast the magnetic connectivity from 1 to 7 days in advance, allowing for sufficient time to decide instrument pointing. Some methods are underway for ranking the individual WSA forecasts, driven by each ADAPT input ensemble map, based on

11 http://heliopropa.irap.omp.eu/ 
the model's recent predictive performance of the observed solar wind (i.e., solar wind speed and interplanetary magnetic field polarity from the LLD data). Once each ensemble member is ranked, it will be used as a weight, allowing for more reliable estimates of the region of the Sun where Solar Orbiter is mostly likely magnetically connected. In addition to comparing WSA solar wind predictions with in situ observations to rank the predictions, further comparisons of WSA model predictions of coronal holes and streamer structure with observations (see Sect. 4.3) are also considered to rank the forecasts.

More elaborate techniques can also be implemented to model the coronal magnetic field continually in a time-dependent manner. The application of the magnetofrictional model introduced by Mackay \& van Ballegooijen (2006) and Yeates et al. (2007) uses synoptic magnetograms to model the evolution of the nonpotential 3D magnetic field configuration of the solar corona. This approach has first proven to be increasingly accurate once the magnetofrictional simulations departs from the potential initial condition and has succeeded in predicting the formation and helicity of low corona structures such as solar filaments (Yeates et al. 2008). At the same time, the variation of the Sun's open magnetic flux (Yeates et al. 2010b) and to a lesser extent the location of CMEs (Yeates et al. 2010a) seem well described. Such an approach needs the input from synoptic magnetograms and Mackay et al. (2016) have addressed how the predictive capabilities significantly improve when a larger portion of the solar surface is updated with recent/real-time magnetograms. Pagano et al. (2018) have coupled this approach with MHD simulations that starts from the initiation of CMEs and addressed in this way how the magnetic flux and plasma are injected in the solar wind at 4 solar radii. In particular, description of the global magnetic field during CMEs will allow to derive how the pre-eruption connectivity is altered by these events.

\subsubsection{Methodology to help with data management onboard}

The connectivity tool can contribute to the prioritisation of data downlink for instruments with internal memories (PHI, EUI, STIX) and selective downlink for EPD and RPW, by helping the instrument teams to make decisions on what datasets have higher scientific importance and are therefore worth being downlinked in priority. These could correspond to, for example, flare source (RS) data connected to an event that has been measured IS or vice versa, or an IS burst mode dataset that is probably linked to a region that has been observed by RS high resolution imagers. Once data have been acquired by Solar Orbiter, the LLD data can be used to improve and/or validate the connectivity diagnostic between a solar feature or event and its impact at the S/C position. For example, STIX-LLD and EUI-LLD will provide cross-checks for the occurrence of flare and CME activity in the low corona, that can be used to flag events registered in the EPDLLD SEPs IS data. By combining both the information provided by the connectivity forecast and the features seen in LLD, instrument teams can make an informed decision on which data to select from onboard storage.

As discussed in Sects. 5.2.2 and 5.4, LLD data will also provide invaluable real-time diagnostics that will help evaluating (and even improving) the quality of the estimated connectivity. For instance PAS-LLD data measurements can be used to provide the actual solar wind speed measured at Solar Orbiter (and hence constraint the shape of the Parker spiral), and HIS-LLD will guide the operators about the compositional and dynamical properties of the plasma (and hence help disambiguate between different candidate sources at the solar surface). PHI-LLD will furthermore be used to improve the coronal magnetic field reconstructions used for estimating connectivity across the corona. Metis-LLD and Solar OrbiterHI-LLD can be used to derive the position of streamers, to estimate CME trajectories, and to measure the wind speed acceleration and propagation profiles above the visible limbs. Even though phenomena that propagate along the limbs are not expected to affect directly the S/C, these observations will provide precious constraints for models that nowcast the state of the eastern limb and eventually to suggest corrections before these streams reach the $\mathrm{S} / \mathrm{C}$ position (due to solar rotation). In the same way, west limb information will provide additional tests for models that may have been used to predict the state of the solar plasma measured by the S/C a few days in advance.

While all these diagnostics will definitely help on the longer term to improve the coronal modelling and connectivity prediction, they also provide valuable context for daily operations. The acquired LLD will help validating the connectivity predictions made by different models and this may lead to both $\mathrm{S} / \mathrm{C}$ pointing updates and adjustment of data downlink priorities.

\subsection{Connectivity tool for science}

The modelling infrastructure developed and implemented for the Connectivity tool will be equally useful for the analysis of the downlinked Solar Orbiter data, and more broadly for postevent analysis based on other (past or future) missions. The tool includes a "science mode" that follows the same strategy than the one described previously for the forecast (or operations) mode, differing only on the combinations of models and datasets available to drive the tool, and being more oriented towards past-event analysis.

By design, the connectivity tool aims at promptly providing Sun-to-S/C connectivity estimation given any complete set of surface magnetogram/coronal field, solar wind model (or asymptotic wind speed values, at minimum) and $\mathrm{S} / \mathrm{C}$ orbital position. As such, the connectivity tool will provide the users multiple ways of relating events occurring in the interplanetary space and at the surface of the Sun (or in close proximity). Multiple S/C analysis, including determining if, e.g., different $\mathrm{S} / \mathrm{C}$ are connected to the same region of the Sun and/or whether they are placed in the same propagation path will also be within close reach.

Even though the connectivity tool already couples together a chain of well identified models (for operational reliability), it should be seen as a modelling framework providing a series of protocols and interfaces that are open to the wider scientific community and ready to interact with different models and databases. As such, it will be possible to drive the connectivity tool (or even just some of its modules) using external data, and equally to serve external models with data generated by any of its different layers (see Sect. 5.2.2). Examples illustrative of this synergy are global heliospheric models, such as EUHFORIA (Pomoell \& Poedts 2018) and ENLIL (Odstrcil \& Pizzo 1999), that can benefit from the connectivity tool performs studies requiring knowledge of the connectivity (and propagation delays) across the solar corona, below their numerical domain. On the other hand, detailed information about the Parker spiral (possibly highly distorted by transient phenomena) in the heliosphere can be fed back to the connectivity tool to improve its capabilites.

Models that describe the formation and acceleration of the solar wind in the corona can also be setup to work synergetically with the tool. Model MULTI-VP (Pinto \& Rouillard 2017), in 
particular, is capable of computing the full acceleration profile of any given solar wind stream along a pre-computed connectivity path, or a bundle of them that fully sample the connectivity uncertainty region. By doing so, MULTI-VP can provide the connectivity tool with detailed information about the propagation of solar wind plasma in all kinds of solar wind stream from the surface to the interplanetary medium, that can differ by several days between from quickly accelerating fast winds streams to very progressively accelerating slow winds. These delays can be fed back to the connectivity tools to improve its estimates. On the other hand, the information provided by the tool can be used to test and validate different heating and acceleration scenarios used by MULTI-VP. The model will also provide detailed phase speed profiles (e.g. sound and Alfvén speeds). Future developments of this and other similar models will include multi-species plasmas and allow for fuller exploitation of Solar Orbiter's IS data, namely that of SWA.

The connectivity tool is implemented as a platform that enables data queries between different solar and space plasma databases using criteria based on connectivity rather than simple spatio-temporal interval searches. Criteria for an array of different phenomena could then be set, from wind plasma propagation to shocks and wavefronts, and SEPs. One implementation of the connectivity tool as an automated remote-to-in situ datacorrelator is already under development within the scope of the STORMS service and of the Propagation Tool (see Sect. 6).

\subsection{Analysis of the uncertainty in magnetic connectivity}

The estimation of surface to S/C connectivity necessarily involves coupling a variety of datasets and models, each of them having their own biases (that cannot always be precisely quantified). It is therefore necessary to devise a method that assesses the quality and the reliability of the connectivity estimations produced by the tool. As explained previously, the connectivity tool goes through two main steps (in its simplest mode of operation): tracing the "Parker spiral" across the interplanetary medium, and tracing the magnetic path across the corona. The former is affected mostly by the uncertainties related with the asymptotic wind speed forecasted at the (future) S/C orbital position, and by the corresponding wind acceleration profile, leading to a continuous region of connectivity at the top of the corona. The latter is more challenging, being affected by the complex topology of the corona itself, such that each connectivity point can be associated with multiple disconnected regions of connectivity at the surface of the Sun. These attributes altogether make a conventional uncertainty and error propagation analysis unfeasible.

So far, we therefore favoured a different strategy, based on computing several estimations of connectivity simultaneously, based on multiple combinations of datasets, models, and/or on different sets of parameters for the same models (e.g., different wind speeds at the $\mathrm{S} / \mathrm{C}$ position). This approach is a form of ensemble modeling, for which the added redundancy (due to making multiple estimations of the same quantity) translates to a better sampling of the region of uncertainty. This procedures has the added interest of letting us have an immediate view of the discrepancies between different methods, which can be more or less severe at different times.

At this point, we have implemented a very simple approach where we consider a distribution of a number of points that are supposed to represent the uncertainty in connectivity between the interplanetary medium and the top of the coronal boundary. We assume a normal distribution in heliocentric longitude and latitude (typically with a larger sigma in the azimuthal direc-
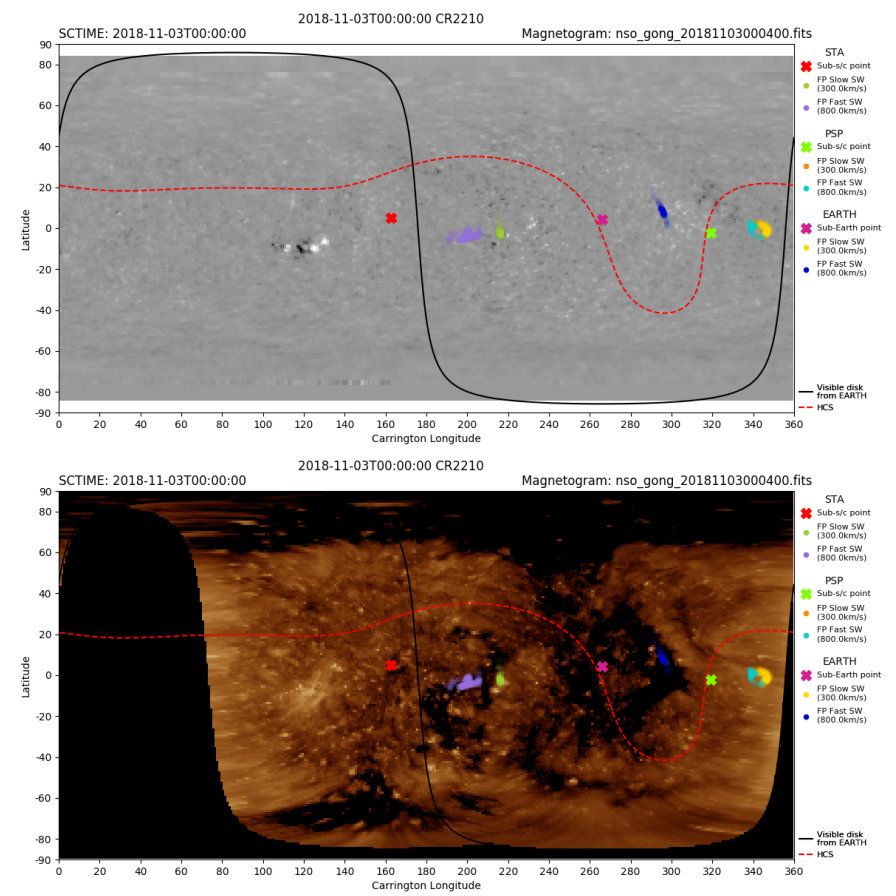

Fig. 14. Connectivity maps produced by the connectivity tool in science mode (for past data) overlayed on a NSO/GONG mangetogram, and on a combined SDO/AIA and STEREO-A/EUVI $193 \AA$ Carrington maps. The symbols have the same meaning as those in Fig. 13, but here we have used the wind speeds measured in situ at L1 and by STEREO-A. Uncertainties intrinsic to the coronal and heliospheric models used are still maintained, giving rise to the corresponding clouds of points in the map.

tion) around the nominal connectivity point. In the simplest configuration of the tool, the latter is simply where the nominal Parker spiral intersects the source surface of the PFSS model. This cloud of points is then mapped down to the photosphere following magnetic field lines in the datacube. Finally, all the distributions are combined into a final connectivity probability at the solar surface, while trying to keep a minimal but meaningful amount of information that allows us to discriminate between different source types (e.g., different types of magnetograms or hypothesis about the solar wind). Figures 13 and 14 show different examples of connectivity probability obtained with this methodology both for forecasts and for past data. Both examples illustrate very clearly that the proximity of a given connectivity path to complex features such as large equatorial streamers and/or the HCS can affect the estimations of connectivity significantly of course. The different S/C shown in Fig. 13 (represented with crosses) all lie quite close to the HCS (red dashed line). The likely connectivity regions for each one of them are reasonably small, but there is a clear separation into two branches, one falling on the northern hemisphere and the other one on the south, with a very large latitudinal separation due to the presence of a wide equatorial streamer. This particularly situation, with the largest separation between disconnected connectivity regions can be expected to occur (perhaps counter-intuitively) during solar minima. Figure 14 shows a different kind of scenario, on which the crossing of a tilted section of the HCS causes the appearance of a continuous arc of connectivity points (violet point cloud). The crossing of equatorial coronal holes away from the HCS can often have the inverse effect, that of concentrating the connectivity points and reducing the associated uncertainties. 


\section{Data analysis tools and Solar Orbiter data}

\subsection{Interoperability through use of standards and calibrated data}

The primary strategy of Solar Orbiter towards data analysis is to produce science data files that in most cases do not require specialized software to analyse. This is accomplished both through the use of well-established standards, and the routine production of fully calibrated higher level data files.

Auchère et al. (2020) discuss the use of well-established standards in the formatting of RS data using the Flexible Image Transfer Standard (FITS; Pence et al. 2010). Walsh et al. (2020) do the same for IS data using the Common Data Format (CDF; Goucher et al. 1994). This embrace of standards goes well beyond the physical structure of the files, but also includes standards for the metadata within the files (MADAWG 2015), following best-established practices within the heliophysics community. This use of standards greatly simplifies the analysis of Solar Orbiter observations by allowing many of the data to be used within already available analysis tools without the need for specialized mission-specific software.

For FITS files, the embrace of standards includes use of the World Coordinate System (see references in Auchère et al. 2020) to describe the pixel (or voxel) coordinates in the data, along with the S/C location at the time of the observation. FITS metadata keywords were also chosen based on common usage in past missions to maximize cross-mission compatibility, and to enable to the use of Solar Orbiter data in already existing tools.

Metadata for Solar Orbiter CDF files were designed based on International Solar-Terrestrial Physics ${ }^{12}$ program guidelines, the Space Physics Archive Search and Extract ${ }^{13}$ dictionary, and the Virtual European Solar and Planetary Access ${ }^{14}$ (VESPA) dictionary (Erard et al. 2018).

The Solar Orbiter instrument teams have committed to providing fully calibrated data, along with higher level data products derived from the directly detected data, further obviating the need for outside researchers to use mission-specific software. Depending on the product, the higher level data files may be in FITS or CDF format (e.g. FITS files containing heliographic synoptic maps) or in a more suitable format for viewing, such as JPEG2000 browse images for use within JHelioviewer (Müller et al. 2017, see also Sect. 6.4.1), MP4 for movies, or PDF for time-series plots.

\subsection{Instrument-provided software}

The launch of Solar Orbiter coincides with a paradigm shift in analysis software for heliophysics data. Up until recently, the most widely used analysis platform has been the commercial Interactive Data Language (IDL) package available from Harris Geospatial Solutions ${ }^{15}$. For primarily remote sensing observations, the IDL SolarSoft ${ }^{16}$ library (Freeland \& Handy 1998) has supported almost all the major heliospheric missions over the last several decades. This trend will continue with missionspecific support in SolarSoft for both the Solar Orbiter and PSP missions.

The major advantage of IDL for the instrument teams is the large availability of useful legacy software. However, some of

\footnotetext{
12 https://spdf.gsfc.nasa.gov

13 http://wWw. spase-group.org

14 http://www . europlanet-vespa.eu/

15 http://www.harrisgeospatial.com/SoftwareTechnology/ IDL . aspx

16 http://wWW. lmsal. com/solarsoft
}

the Solar Orbiter instrument teams are starting to evaluate the use of Python for software development, including data analysis. Python is seen as the wave of the future, not only for heliophysics but for science in general. The use of Python for Solar Orbiter is assisted by the SunPy ${ }^{17}$ project (SunPy Community et al. 2015), which aims to do for Python what SolarSoft does for IDL, provide an integrated analysis environment for heliophysics data. The Connectivity Tool described in Sect. 5.1 was mostly written in Python and exploits the SunPy package.

Aside from the specific language used for software development, the importance of version control has become better appreciated within the heliophysics community. Many packages written in Python, including SunPy, use GitHub ${ }^{18}$ for version control and distribution to the community. Solar Orbiter instrument teams that plan to distribute software to the community in Python in conjunction with SunPy also plan to use GitHub.

Along with the calibrated data files, the Solar Orbiter teams will also make publicly available the software used to generate the calibrated files from the lower level data. This will allow alternative calibration techniques to be explored, both from within the instrument teams and without, as well as document how the calibration was applied. The FITS or CDF metadata will include the version of the applied calibration, and the instrument teams will make public their calibration history, so that users can know whether they are using data with the most up-to-date calibration.

There are cases when simply providing calibrated data is insufficient, and additional software must be provided by the instrument teams to aid the user in analysing the data. A good example of this is the spectral information returned by the SPICE instrument (SPICE Consortium 2020). The SPICE team will provide IDL software to assist researchers to fit emission lines, based on software used for earlier missions, and to derive information from combinations of spectral lines, such as differential emission measures (DEMs), abundances, and maps of first ionization potential (FIP) effects. Similarly, the STIX (Krucker et al., in prep.) instrument team will provide IDL tools for data analysis based on RHESSI (Lin et al. 1998) heritage.

\subsection{Virtual observatories}

The rise of virtual observatories (VOs) in astronomy and heliophysics for data discovery and distribution has greatly improved the science returned by missions. Solar Orbiter is fully committed to making data available to the existing relevant VOs. Release of the data to the VOs will occur once the data rights policy has been satisfied and the data can be made public.

Remote sensing FITS files will be made available to the Virtual Solar Observatory ${ }^{19}$ (VSO; Hill et al. 2004). The VSO system consists of distributed data archives linked together through a uniform interface for searches and data requests. Interfaces to VSO exist in both IDL/SolarSoft and Python/SunPy, as well as through the web. Solar Orbiter FITS data files will be made available to the VSO through the ESA Solar Orbiter Science Archive (SOAR) at ESAC, Spain (Sanchez et al., in prep.). This will include uncalibrated Level 1 files, calibrated Level 2, and some derived Level 3 products such as mosaics, FIP maps, heliographic maps, and time-distance plots ("J-maps"). Some nonFITS Level 3 products may also be served through the VSO as appropriate.

\footnotetext{
17 https://sunpy.org

18 https://github.com

19 https://virtualsolar.org
} 


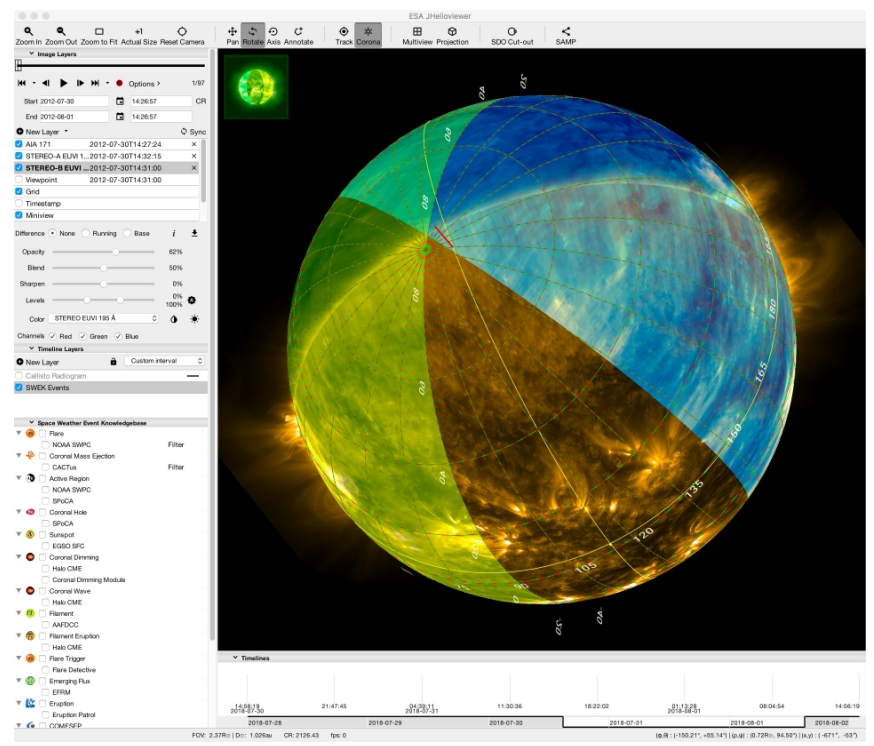

Fig. 15. JHelioviewer program, showing a combination of simultaneous images from SDO, STEREO-A, and STEREO-B for a date in 2012, viewed from a high latitude. Note the areas of overlap between the three views.

\section{4. $3 D$ visualization tools}

In order to exploit the Solar Orbiter data and combine it with data from other missions, the community has developed powerful 3D visualisation tools that are described in this section. We provide a list of the relevant references to direct the interested reader to a more detailed description of these tools.

\subsubsection{JHelioviewer}

JHelioviewer (Müller et al. 2017) is a Java-based visualization tool for solar image data. Together with the web application ${ }^{20}$, it is part of the joint ESA/NASA Helioviewer Project ${ }^{21}$ (see Fig. 15). The large data volumes from modern solar observatories are reduced to manageable levels by compressing images using the JPEG2000 standard (Taubman \& Marcellin 2002), and serving them through the JPIP streaming protocol. This optimizes the delivery of usable resolution within the selected region of interest while still delivering the full (compressed) resolution in the background. In addition, JHelioviewer supports loading some calibrated datasets in FITS format and will support the Solar Orbiter metadata standards.

The current capabilities of JHelioviewer include the visualisation of time series of image data from multiple sources (e.g. SDO, SOHO, PROBA2) in a single consistent fashion, movie controls, an interface to the SDO cut-out service. It also allows a visualisation of multi-viewpoint data in a single $3 \mathrm{D}$ scene (e.g. STEREO), combined with optional planar views of off-limb data. JHelioviewer can display running and base difference images in real time and allows overplotting of potential-field source-surface (PFSS) magnetic field extrapolation models. Recent additions to the tool provides an interface to visualise timelines of $1 \mathrm{D}$ and 2D data (e.g. GOES X-ray fluxes, radio spectrograms).

JPEG2000 images from Solar Orbiter for use in the JHelioviewer will be produced at and served through the Solar

\footnotetext{
20 https://helioviewer .org

21 http://wiki.helioviewer.org
}

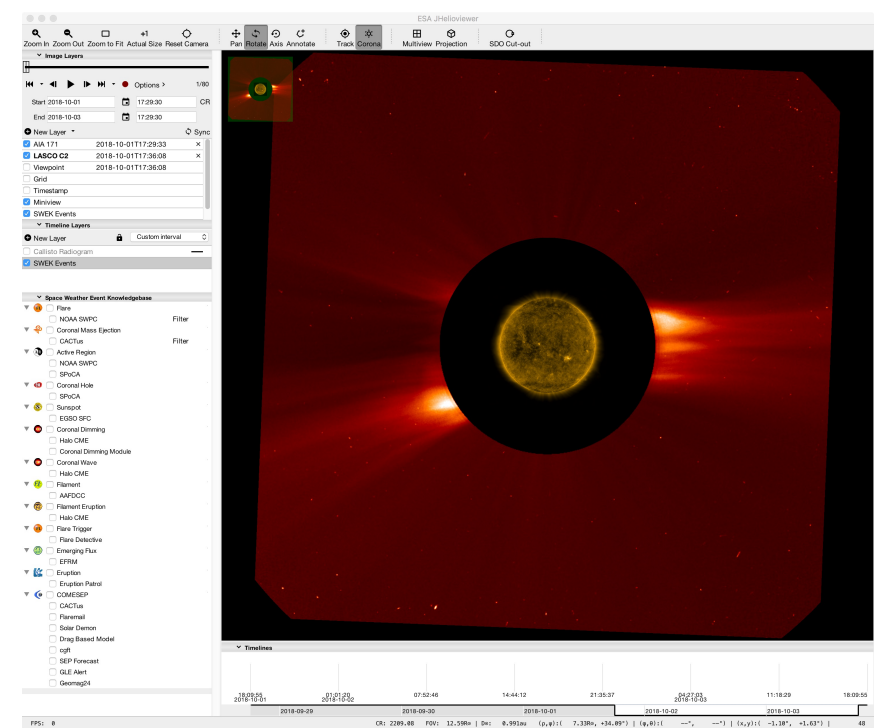

Fig. 16. Combination of SDO/AIA $171 \AA$ And SOHO/LASCO C 2 white light images, demonstrating the ability of JHelioviewer to merge disk imagery with coronagraph data such as those from the Solar Orbiter Metis instrument.

Orbier Science Archive (SOAR). Low latency versions will also be made available for short-term planning purposes, with appropriate restrictions applied to satisfy the data rights policy (Sanchez et al., in prep.) (see Fig. 16).

JHelioviewer can also receive data over the IVOA SAMP protocol $^{22}$. One application is the serving of calibrated science data directly from the ESAC archive. The program state itself can also be transmitted in this manner, opening possibilities for remote collaboration. The connection to JHelioviewer over SAMP can also be initiated from a web page, which makes this feature particularly useful for catalogues of events or models ${ }^{23}$.

EUHFORIA model results have recently been integrated in JHelioviewer and will be available on a daily basis to complement the Solar Orbiter data and both will be visualized side by side through the JHelioviewer interface (see Fig. 17 and Sect. 6.4.1). EUHFORIA will model self-consistently the propagation of the solar wind, the deformation, deflection and erosion of CMEs evolving in the inner heliosphere. It will help characterise the magnetosheaths of CMEs and clarify the role of CME-CME interactions in enhanced SEP production. The EUHFORIA model already contains trajectory information of Solar Orbiter. The model can provide the magnetic connection of the $\mathrm{S} / \mathrm{C}$ to the photosphere. As already discussed, EUHFORIA datacubes will also be made available to the connectivity tool, so they can be used directly there. There are also plans to create a website service from the Connectivity Tool that can be interrogated by JHelioviewer as well the other tools discussed in the next section.

\subsubsection{DView}

3DView ${ }^{24}$ (Génot et al. 2018) is a Java-based 3D visualization tool developed by CDPP, the French Plasma Physics Data Center. The initial focus of the tool was to render S/C and planetary

\footnotetext{
22 http://www . ivoa.net/documents/SAMP/

23 Demos at http://swhv . oma.be/test/samp/

24 http://3dview.cdpp.eu
} 


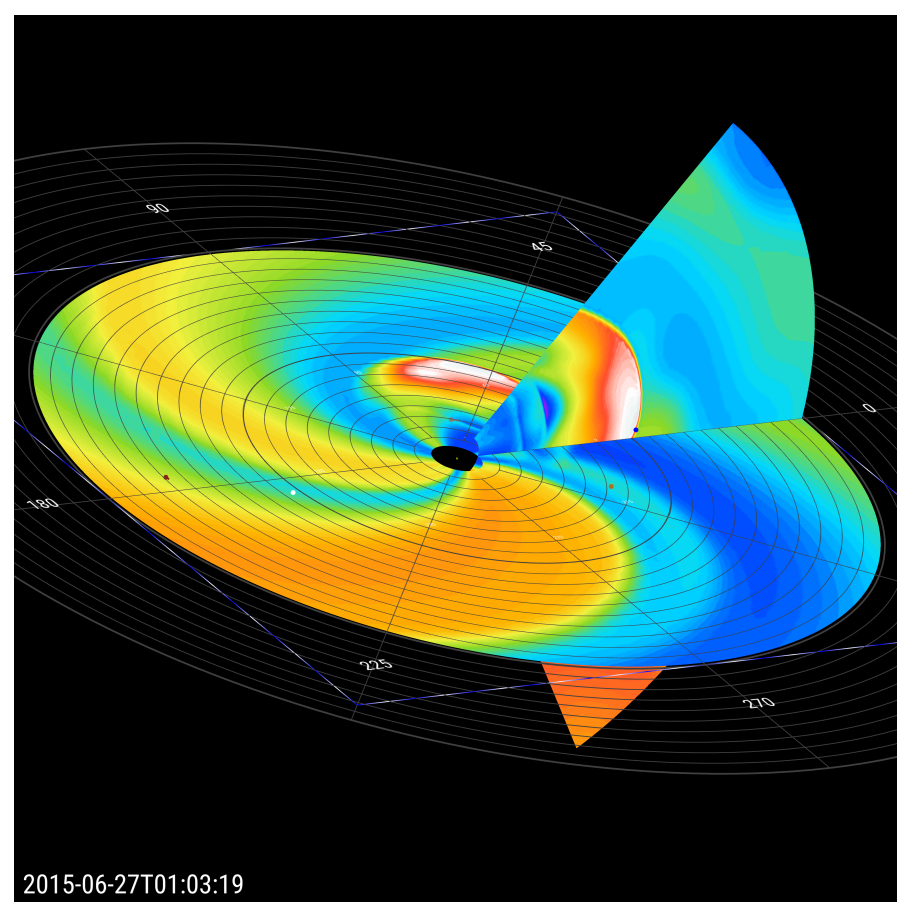

Fig. 17. Equatorial and Earth-meridional solar wind radial speed modelled by EUHFORIA as displayed by JHelioviewer. Mercury, Venus, Earth, STEREO-A, and Mars are displayed in HEEQ (Heliocentric Earth Equatorial) reference frame.

body trajectories and attitudes, based on SPICE kernels (data files for the NAIF SPICE toolkit (Navigation and Ancillary Information Facility; Spacecraft, Planet, Instrument, Camera, Events; Acton 1996; Acton et al. 2018)), in 3D maneuverable scenes from which movies could be extracted. Numerous extensions led to enhanced functionalities such as the ability to display numerical models (planetary boundaries, magnetic field lines, CME fronts, ... ), observational data from in situ instruments onboard space missions and simulation runs from global (MHD, hybrid) codes. These various data can be uploaded by the user, or directly accessed (AMDA see Sect. 6.6, CDAWeb, Cluster Science Archive, LatHyS database) thanks to standardized protocols (SAMP, EPN-TAP) and web-services. Figure 18 illustrates some of these functionalities by displaying the orbits of Solar Orbiter and PSP together with their magnetic connection to the Sun; at $30 R_{\mathrm{S}}$ from the Sun the radial velocity modelled by MULTI-VP (Pinto \& Rouillard 2017) is colour-coded. Other functionalities include, for example, displaying radial and Parker field line connectivity for S/C onto Carrington maps, representation of the Heliospheric Current Sheet (Pei et al. 2012) or instrument fields of view. Screen shots and video captures are possible. Future implementations will take benefit of the results of the magnetic connectivity tools discussed in Sect. 5.1.

\subsubsection{SunGlobe}

SunGlobe is an IDL-based tool in SolarSoft which uses the same JPEG2000 images as JHelioviewer but with a different focus ${ }^{25}$. JHelioviewer is designed primarily for data discovery and analysis, while the intent of SunGlobe is to assist in target selection when planning future observations. For this reason, the way that images are combined together is different from that of $\mathrm{JHe}$ -

\footnotetext{
${ }^{25}$ SunGlobe is developed by W. T. Thompson: https://hesperia.
} gsfc.nasa.gov/ssw/gen/idl/sunglobe/sunglobe.pro

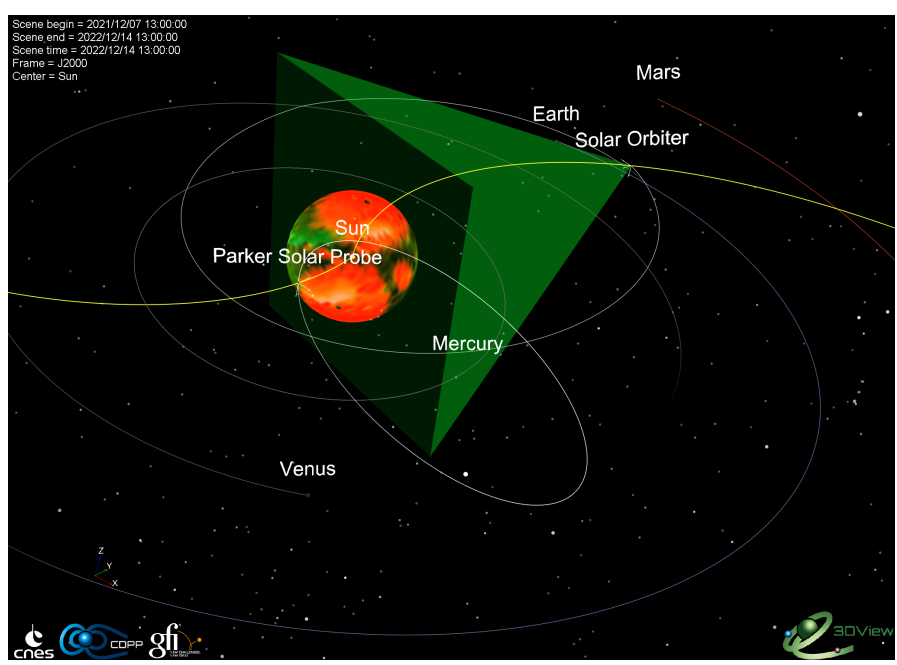

Fig. 18. Heliospheric view in December 2022 with 3DView. The Parker field lines (at $V=500 \mathrm{~km} \mathrm{~s}^{-1}$ ) connected to Solar Orbiter and PSP are shown in yellow. The surface around the Sun (here at $30 R_{\mathrm{s}}$ ) is the colour coded solar wind velocity output from the MULTI-VP code (Pinto \& Rouillard 2017). The Solar OrbiterHI field of view is displayed in green.

lioviewer. Instead of generating movies of cotemporal images from multiple sources, SunGlobe uses single images, and these images can be at different times - differential rotation is used to match all images to a single observation time in the future. This allows images from multiple dates to be combined together to give a sense of the state of the whole Sun, both near and far sides.

Figure 19 shows an example of using SunGlobe to select the $\mathrm{S} / \mathrm{C}$ pointing for a given configuration of the SPICE instrument, together with the EUI and PHI high resolution fields-of-view. SunGlobe uses the ephemeris data for Solar Orbiter (or other observatory if appropriate ephemeris files are provided) to determine the viewing geometry at the planned observing time. Users can also interactively change the orientation, viewing distance, and pan and zoom. Other plotting capabilities include PFSS magnetic field extrapolations, and the track of the S/C subsurface point as a function of time. The current implementation of SunGlobe uses a simple PFSS model combined with a Parker spiral extrapolation to estimate the magnetic connection point on the surface. Future implementations will use the results of the magnetic connectivity tools discussed in Sect. 5.1.

\subsection{J-map visualisation tools}

Software made available in open libraries such as SolarSoft or via web-based interfaces allow the community to visualise and manipulate height-time maps. We describe these J-map visualisation tools in this section.

\subsubsection{SATPLOT}

SATPLOT software was developed to create and analyse such elongation versus time plots. The tool uses a library of cylindrical maps of the data for each S/C's panoramic field-of-view. Each map includes data from three SECCHI white-light telescopes (the COR2 coronagraph and both heliospheric imagers) at one time for one $\mathrm{S} / \mathrm{C}$. The maps are created using a Plate Carree projection, optimized for creating the elongation versus time plots. The tool can be used to analyse the observed tracks 


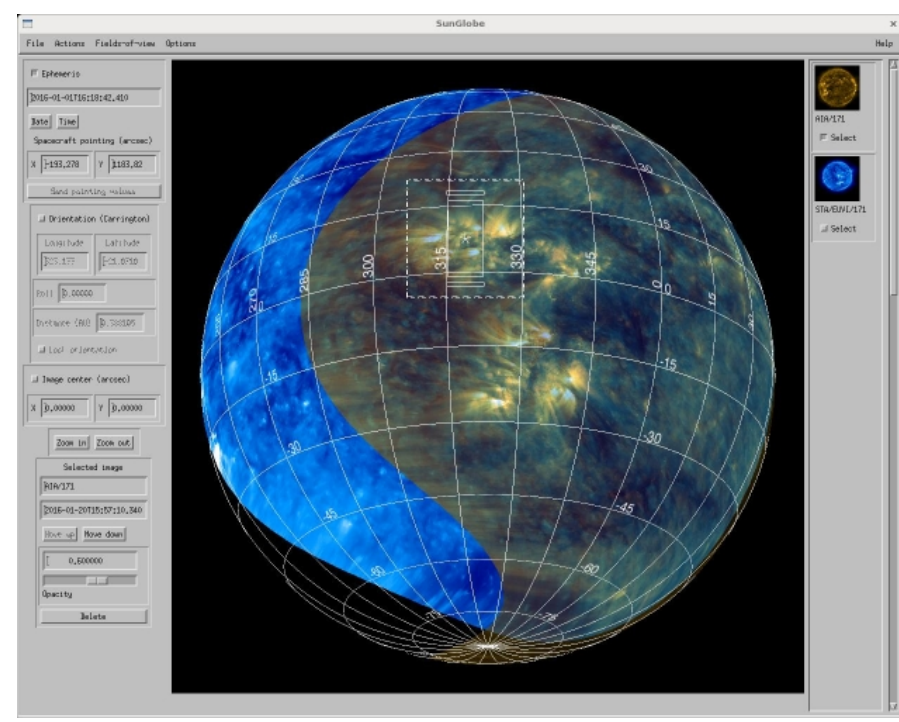

Fig. 19. SunGlobe program, showing the fields-of-view of SPICE, EUI, and $\mathrm{PHI}$ for a selected $\mathrm{S} / \mathrm{C}$ pointing.

of features seen in the maps, and the tracks are then used to extract information, for example, on the angle of propagation of the feature.

\subsubsection{Propagation tool}

The Propagation Tool ${ }^{26}$ (Rouillard et al. 2017) exploits heliospheric imagery as observational support to connect solar imagery with in situ measurements in an interactive manner. The web-based interface offers an integrated research environment to study the evolution of coronal and solar wind structures, such as CMEs, CIRs and SEPs. These structures can be propagated from the Sun outwards to or alternatively inwards from planets and $\mathrm{S} / \mathrm{C}$ situated in the inner and outer heliosphere. The tool was created to provide a simple way to propagate heliospheric structures in order to connect RS observations with in situ measurements. It offers an interactive manipulation of J-maps based on images taken by STEREO but along the ecliptic plane only. The user can define the properties (speed, direction) of hypothetical CMEs and compare predicted trajectories with traces in J-maps. The tool offers also access to predefined catalogues of CME trajectories (such as those created by the HELCATS project ${ }^{27}$ ) or the possibility to fit traces observed in J-maps directly. The same functionalities can be exploited to locate CIRs and their arrival times. Once a propagation has been carried out with the tool and a launch time has been estimated at the Sun, the user can then navigate between different databases and other tools such as AMDA or JHelioviewer webpage. For SEPs studies the tool provides estimates of $\mathrm{S} / \mathrm{C}$ magnetic connectivity to the visible solar disk, locations of flare occurrence, browsing of simulation results of the solar corona (PFSS, MHD) and access to the results of $3 \mathrm{D}$ reconstructions of shock waves. Future developments include the incorporation of J-maps from future heliospheric imagers such as WISPR (Vourlidas et al. 2016) and Solar OrbiterHI (Howard et al. 2020) as well as the possibility of visualising J-maps along position angles situated outside the ecliptic plane.

\footnotetext{
${ }^{26}$ http://propagationtool.cdpp.eu

27 ww. helcats-fp7.eu
}

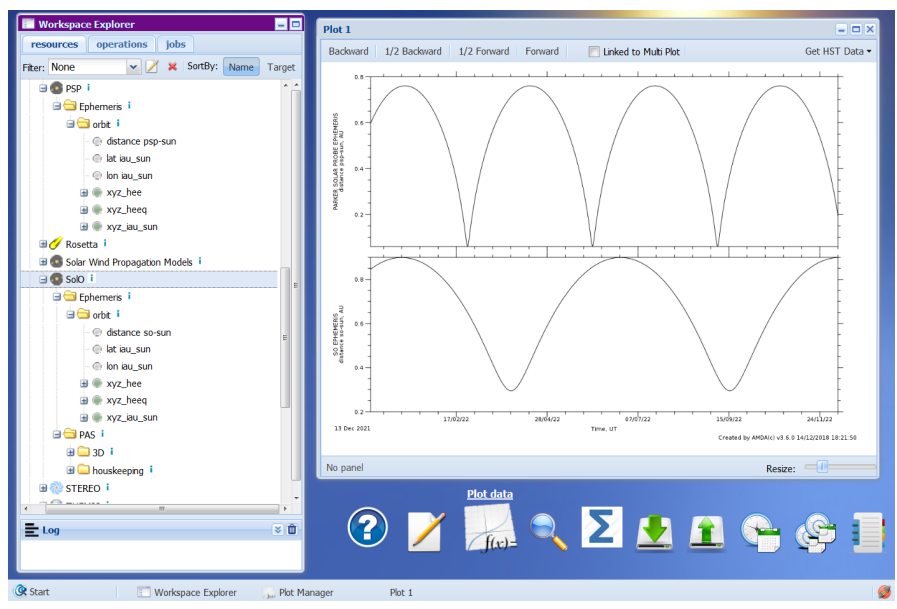

Fig. 20. AMDA interface with a portion of the data tree on the left and a display of Solar Orbiter and PSP distances to the Sun on the right. The icons in the bottom part of the window illustrate the different analysis and management capabilities (data mining, cataloguing, statistics, ...).

\subsection{IS visualisation and data mining tools}

$\mathrm{AMDA}^{28}$, Automated Multi Dataset Analysis (Jacquey et al. 2010; Génot et al. 2010), is developped by the CDPP for more than 12 years and specializes in data visualisation and analysis of plasma physics data, from ground-based facilities, space missions or simulations and models. The software enables easy manipulation of parameters (scalars, vectors or spectrograms) which may be combined directly in the browser interface (see an illustration on Fig. 20 here captured from a Firefox window). Mathematical criteria can be applied on vast volume of data to produce event lists or catalogues. Users have their own workspaces such that all sessions (and associated new parameters, catalogues, plot layouts, ...) are logged and saved on the long term. This simplicity helped popularizing the tool which is used by researchers and students alike. A large variety of data are available either directly (duplication from NASA/PDS for planetary missions, or Cluster Sience Archive, for instance), through web-services (CDAWeb, simulations, ...), or by user upload. On the VO side, SAMP is enabled and all AMDA data are also accessible via EPN-TAP as can be seen on the VESPA portal. In 2014, the ability of AMDA to handle heterogeneous datasets has decided the Rosetta Plasma Consortium leads to ask CDPP to provide a dedicated portal to the team; AMDA has evolved accordingly to allow the Rosetta Plasma Consortium (RPC) group to access their proprietary data during all operations before the ESA/PSA archive went public. This facility greatly helped the team analyse, intercalibrate and share their datasets. Numerous publications were issued from this collaboration between the CDPP and RPC. A similar approach could be adopted for the IS instruments of Solar Orbiter. A prototype is currently developed to exchange test data within the SWA/PAS team (see the PAS directories on the bottom left of Fig. 20).

\section{Modeling support for the Solar Orbiter Space Weather Laboratory}

As discussed elsewhere in this volume, the science operations of Solar Orbiter differ significantly from past solar missions. The remote sensing payload operates only during the RSW

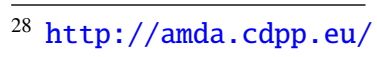


which constitute only 30 days during the 168-day orbit, while the IS data collection will be interrupted during data downlinks and possibly during the operation of the remote sensing instruments. Since Space Weather operations rely on continuous solar monitoring, the intermittency of the Solar Orbiter observations reduces considerably the space weather operational value of the mission compared to missions like SOHO or STEREO.

Solar Orbiter will however significantly contribute to research in space weather issues given its unique trajectory that takes the S/C outside the ecliptic plane, away from the Sun-Earth line, and near the fringes of the outer solar corona with a highly complementary payload to boot. On the other hand, the short RSWs require careful target selection to optimize the studies of solar transient activity. The latter comprise half of the Solar Orbiter science objectives and hence are too important in the success of the mission to leave progress on them to serendipity alone. Here, space weather-focused tools developed to improve forecasting of transient activity can be deployed to great benefit in conjunction with the other tools described elsewhere in this paper.

In the rest of the section, we discuss briefly how space weather efforts can inform and be informed by the Solar Orbiter data analysis and modeling activities. We illustrate that Solar Orbiter will be tremendously important for space weather science even though it is not a surveillance/monitoring platform. We refer to this concept as the Solar Orbiter Space Weather Laboratory. Space constraints do not allow us to cover all possible tools, models and projects that could be incorporated in the MADAWG and SOC activities on the subject. Instead, we provide some indicative examples to attract the interest (and contributions) of the research and operational community in the hope of strengthening the Space Weather value of the mission in the years to come.

\subsection{Space weather tools for Solar Orbiter science operations}

There has been considerable progress in the last decade in the development of tools to forecast space weather activity. Although these tools are meant for improving space weather operations at Earth, some of the tools could be equally helpful in the science operations of Solar Orbiter. The latter require target selection and observational program definition weeks in advance and only, relatively small, targeting corrections are permitted up to 2-3 days before execution, at best. Given these restrictions and the intermittent nature of solar activity, medium- to shortterm forecasts of flaring/erupting activity could play an important role in target and observing program selection.

CME and SEP forecasting generally kicks off after the detection of an eruption and hence provides only real-time or near real-time predictions, e.g., COMESEP (Crosby et al. 2012), HESPERIA (Núñez et al. 2018). The Forecasting Solar Particle Events and Flares (FORSPEF) tool (Anastasiadis et al. 2017) provides forecasts of solar flares, including the occurrence and speed of accompanying CMEs and the likelihood of an SEP event, using diagnostics extracted from full-disk magnetograms. Predictions are currently provided for the next $24 \mathrm{~h}$, which is a bit better than near real-time but still insufficient for Solar Orbiter planning.

Building on FORSPEF, the Advanced Solar Particle Event Casting System (ASPECS; currently under development) will provide advanced warnings for SEPs over a set of windows ( $4 \mathrm{~h}-, 12 \mathrm{~h}-, 24 \mathrm{~h}$ - to $48 \mathrm{~h}$ ) extending also to $72 \mathrm{~h}$ in advance of the identification of any eruptive event on the solar disk. In doing so, ASPECS incorporates the Spaceweather HMI Active Region
Patch (SHARP) in its solar flare forecasting scheme, facilitating the need for accurate solar flare forecasts closer to the solar limb. Coupling of a physics-based (SOLPENCO; Aran et al. 2008) and an empirical data driven approach (Kahler \& Ling 2017) will provide the expected SEP time profile in energies $(E>10-$; $>30-$; >100- and >330 MeV).

Solar Orbiter and SEP forecasting tools have a mutually beneficial relation. On one hand, the application of such SEP forecasting tool to Solar Orbiter operations is straightforward since from the SHARP identifications a forecasting of the radiation environment 3 days in advance could be achieved. This will also include the expected SEP time profile. Thereby, planning teams can evaluate the situation at hand and decide how to proceed. On the other hand, the datasets provided by Solar Orbiter would be uniquely suitable to test the empirical relations and corresponding predictors that form the basis for forecasting/nowcasting tools.

Forecasting of flares is another space-weather area of interest to Solar Orbiter. Numerous flare prediction schemes exist in the literature, (i.e. Huang et al. 2018; Laurenza et al. 2018; Leka et al. 2018; Liu et al. 2017; Nishizuka et al. 2018), to name a few recent ones. Some involve members of the MADAWG team, such as the EU Flare Likelihood and Region Eruption Forecasting project (FLARECAST; Georgoulis et al. 2017). FLARECAST provides a unique toolbox of readily accessible, opensource algorithms to extract more than 100 quantitative diagnostics (many of them in both "rojected" and "unprojected" versions) from solar line-of-sight and vector magnetograms, such as magnetic PILs, velocity shear, electric currents and magnetic helicity, among many others. These quantitative properties can then be used to estimate the flaring potential of a region (e.g. Kontogiannis et al. 2017, 2018). The forecast window of the various FLARECAST scheme(s) can be adjusted from several $(<10)$ to $48 \mathrm{~h}$. A robust performance verification, currently under way, will indicate the optimal window(s). However, to credibly extend the forecast windows beyond $48-72 \mathrm{~h}$ would require knowledge of subsurface processes (i.e., emergence of new active regions (e.g. Reinard et al. 2010) and farside imaging (e.g. Lindsey \& Braun 1997). In addition, extreme solar flares (class $\mathrm{X}+$ ) elude currently available alogrithms (e.g. Barnes et al. 2016), probably due to their scarcity. Further research, including the Solar Orbiter observations, to improve FLARECAST and other efforts at work.

Assuming that such developments are forthcoming, the application to Solar Orbiter seems straightforward: Earth-based photospheric magnetograms, updated as often as possible, are fed to a set of these forecasting tools to identify likely erupting targets on the Solar Orbiter visible disk for the next week or so. When the $\mathrm{S} / \mathrm{C}$ is on the far side, the magnetograms could be advanced using flux transport schemes such as ADAPT, described in Sect. 4.1.2. If available, PHI magnetograms (and possibly EUI images), from outside the RSW, could be used to augment the reliability of predictions. The science planning teams will then evaluate the forecasted targets and choose whether to alter the baseline program or not. At a minimum, this exercise will provide an excellent testbed to evaluate/validate the forecasting success of the various schemes, which will help in further development.

Magnetofrictional simulations (Mackay et al. 2011) can efficiently simulate the evolution of active regions (Gibb et al. 2014; Yardley et al. 2018), where a series of observed magnetograms are used to drive the evolution of the 3D magnetic field configuration. By analysing the force balance evolution, magnetofrictional simulations offer a novel approach to predict the lift off 

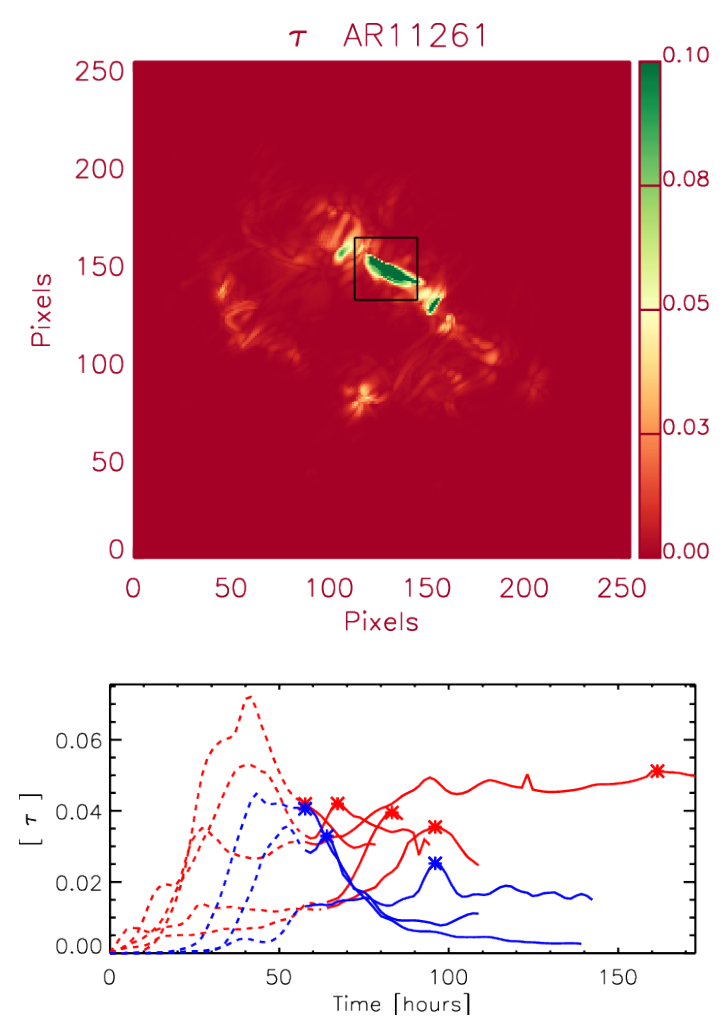

Fig. 21. Upper panel: map of $\tau$ for the active region AR11261 near the time of its observed eruption. Lower panel: evolution of $\tau$ for 8 active regions examined. In five of them an eruption was observed (AR11261, AR11561, AR11504, AR11437, AR11680 - red lines) and in three of them no eruption was observed (AR11480, AR11813, AR12455 - blue lines).

of magnetic flux ropes. Some preliminary studies show that a simultaneous estimation of the twist of the magnetic field, the integral of the vertical component of the Lorentz force and its heterogeneity, condensed in a parameter called $\tau$, can discern eruptive and non eruptive active regions. The upper panel in Fig. 21 shows a colour map of $\tau$ near the time of eruption of the active region AR11261. Such a parameter shows a peak at the location where observations (Rodkin et al. 2017) have identified the formation of a magnetic flux rope and its subsequent ejection. Extending this approach to multiple active regions, it is found that this metric, $\tau$, can be used to identify eruptive active regions. The lower panel in Fig. 21 shows the time evolution of the $\tau$ parameter for a set of 8 active regions. The five eruptive active regions(red lines) show a significantly different trend from the three non-eruptive regions (blue lines). Such simulations could be use to fine-tune the target selection for Solar Orbiter eruptive studies.

Simulations of the background solar corona are equally relevant for space weather applications, as it is, on one hand, the medium through which transients such as CME propagate through and evolve, and on the other a source of space weather effects on its own (such as co-rotating interaction regions).

Great efforts have also been made in recent years to improve the quality, reliability and computational efficiency of such models, in parallel to semi-empirical predictive laws. New modeling approaches (e.g., SWiFT/MULTI-VP) aim at providing datadriven and physics-based forecasts of the distributions of fast and slow wind flows, together with the associated densities and dynamical pressures, while keeping real-time capabilities (Pinto \& Rouillard 2017). Currently, full sets of bulk physical parame- ters of the solar wind (surface to $1 \mathrm{AU}$ ) can be provided up to a few days in advance.

\subsection{Solar Orbiter Contributions to space weather research}

Several outstanding questions in space weather research, such as CME initiation, propagation and interaction with the ambient environment, stand to benefit greatly from Solar Orbiter's unique viewpoints and complementary payload. The extended coverage of the photospheric magnetic field and the detailed observations of the CME source regions will improve two key Space Weather issues: (1) modeling of the solar wind (discussed in Sect. 4.1.2) and (2) understanding of CME initiation. Data from PHI, EUI, and Metis will provide a unique insight on the magnetic field and plasma evolution during magnetic flux rope and sheared arcades ejections. These events present a challenge for modelling as different physical regimes, time and spatial scales, are involved, when the free magnetic energy is built up prior to the eruption and it is quickly and suddenly released. To this end, simulations based on the evolution of magnetofrictional models coupled with MHD simulations provide an interesting approach, as it can simultaneously account for observational constraints ranging from the built-up phase (mostly PHI) to the eruption and propagation of magnetic flux ropes (EUI and Metis). In particular, in the magnetofrictional approach a continuous time series of 3D Non-Linear Force Free field configurations is derived from a corresponding time series of photospheric vector magnetograms. Numerous works showcase the robustness of this approach in describing the formation and the slow, quasi-static evolution of magnetic structures, such as magnetic flux ropes in active regions, as well as in describing the evolution of the global corona. Ongoing efforts are focusing on using the properties of the evolving magnetic field to predict the onset of magnetic flux rope ejections. When magnetofrictinonal and MHD simulations are coupled, a pre-eruptive but unstable magnetic configuration is transferred from one another as initial condition and a typical plasma distribution is assumed (Pagano et al. 2013a). In this approach, MHD simulations of CMEs can be used to infer the properties of the coronal plasma through the kinematic properties of the CME propagation (Pagano et al. 2013b; Rodkin et al. 2017), or by means of a more advanced diagnostic when we synthesize remote sensing measurements (Pagano et al. 2014, 2015).

\section{Conclusions}

The coming years for heliophysics research promise to unravel a number of outstanding mysteries about our Sun and how it couples to the heliosphere. We described the current state of the art in data-analysis tools and models that can exploit and benefit from the type of data that will be obtained by Solar Orbiter with a particular focus on tools that ease comparison of RS with IS data. The on-going effort by ESA's MADAWG aims at providing the tools necessary to support mission operations during data acquisition and in the process of on-board data management and downlink to Earth. As our understanding of the corona improves over the next years, these tools and techniques will no doubt evolve further strengthening our procedure and methodology. A number of existing assets that have greatly benefited from the last decade of space missions are currently being modified so that Solar Orbiter data can be directly integrated in them. This requires that data formats follow international standards and exchange protocols that have also been integrated by the MADAWG. Several models not discussed here are currently being developed to integrate data from more 
instruments on Solar Orbiter and other observatories such as PSP and ESA's ASPIICS instrument on PROBA-3. Little work has been done so far at preparing ground-based support for Solar Orbiter and this constitutes one our next challenges. These are exciting opportunities for young researchers in heliophysics that are always welcome to join our dynamic working group.

Acknowledgements. Solar Orbiter is a joint ESA and NASA mission. A Vourlidas' Solar Orbiter effort is supported by NRL grant N00173-16-1-G029. P. Pagano would like to thank D. H. Mackay and S. L. Yardley for their valuable contributions, the European Research Council (ERC) under the European Union Horizon 2020 research and innovation program (grant agreement No. 647214), and the DiRAC Data Centric system at Durham University, operated by the Institute for Computational Cosmology on behalf of the STFC DiRAC HPC Facility (http://www.dirac.ac.uk). This equipment was funded by a BIS National E-infrastructure capital grant ST/K00042X/1, STFC capital grant ST/K00087X/1, DiRAC Operations grant ST/K003267/1 and Durham University. DiRAC is part of the National E-Infrastructure. A. Rouillard, V. Génot, M. Janvier, Elie Soubrier, F. Auchère, E. Buchlin and E. Pariat acknowledge support from the French space agency (Centre National d'Études Spatiales; CNES; https://cnes. $\mathrm{fr} / \mathrm{fr}$ ) that funds activity in plasma physics data center (Centre de Données de la Physique des Plasmas; CDPP; http://cdpp.eu/) and the Multi Experiment Data and Operation Center (MEDOC; https: //idoc.ias.u-psud.fr/MEDOC), and the space weather team in Toulouse (Solar-Terrestrial Observations and Modelling Service; STORMS; http:// storms-service.irap.omp.eu/). This includes funding for Gaia-DEM, the data mining tools AMDA (http://amda.cdpp. eu/), CLWEB (clweb.cesr. $\mathrm{fr} /$ ) and the propagation tool (http://propagationtool.cdpp.eu). R Pinto, M. Lavarra, Y. Wu and A. Kouloumvakos acknowledge financial support from the ANR project SLOW_SOURCE No. ANR-17-CE31-0006-01, ANR project COROSHOCK No. ANR-18-ERC1-0006-01 and FP7 HELCATS project https://www.helcats-fp7.eu/ under the FP7 EU contract number 606692. A. Warmuth acknowledges the support by DLR under grant No. 50 QL 0001. The STEREO SECCHI data are produced by a consortium of RAL (UK), NRL (USA), LMSAL (USA), GSFC (USA), MPS (Germany), CSL (Belgium), IOTA (France) and IAS (France). The ACE data were obtained from the ACE science center. The WIND data were obtained from the Space Physics Data Facility. Javier Rodriguez-Pacheco acknowledges Spanish Project: FEDER/MCIU-AEI/Project ESP2017-88436-R.

\section{References}

Acton, C. H. 1996, Planet. Space Sci., 44, 65

Acton, C., Bachman, N., Semenov, B., \& Wright, E. 2018, Planet. Space Sci., 150,9

Afanasiev, A., Vainio, R., Rouillard, A. P., et al. 2018, A\&A, 614, A4

Albert, K., Hirzberger, J., Busse, D., et al. 2018, in Software and Cyberinfrastructure for Astronomy V, Proc. SPIE, 10707, 1070700

Amari, T., Boulmezaoud, T. Z., \& Aly, J. J. 2006, A\&A, 446, 691

Amari, T., Canou, A., Aly, J.-J., Delyon, F., \& Alauzet, F. 2018, Nature, 554, 211

Anastasiadis, A., Papaioannou, A., Sandberg, I., et al. 2017, Sol. Phys., 292, 134

Antonucci, E., Romoli, M., Andretta, V., et al. 2020, A\&A, 642, A10 (Solar Orbiter SI)

Aran, A., Sanahuja, B., \& Lario, D. 2008, AdSpR, 42, 1492

Arge, C. N., Luhmann, J. G., Odstrcil, D., Schrijver, C. J., \& Li, Y. 2004, J. Atm. Sol. Terrest. Phys., 66, 1295

Arge, C. N., Henney, C. J., Koller, J., et al. 2010, Sol. Wind Conf., 1216, 343

Arge, C. N., Henney, C. J., Hernandez, I. G., et al. 2013, Sol. Wind, 13, 11

Aschwanden, M. J. 2013, ApJ, 763, 115

Aschwanden, M. J., \& Boerner, P. 2011, ApJ, 732, 81

Aschwanden, M. J., Wuelser, J.-P., Nitta, N. V., Lemen, J. R., \& Sandman, A. 2009, ApJ, 695, 12

Auchère, F. 2005, ApJ, 622, 737

Auchère, F., Cook, J. W., Newmark, J. S., et al. 2005, AdSpR, 35, 388

Auchère, F., Andretta, V., Antonucci, E., et al. 2020, A\&A, 642, A6 (Solar Orbiter SI)

Aulanier, G., Démoulin, P., Mein, N., et al. 1999, A\&A, 342, 867

Bai, X. Y., Deng, Y. Y., Teng, F., et al. 2014, MNRAS, 445, 49

Barbey, N., Auchère, F., Rodet, T., \& Vial, J.-C. 2008, Sol. Phys., 248, 409

Barbey, N., Guennou, C., \& Auchère, F. 2013, Sol. Phys., 283, 227

Barnes, G., Leka, K. D., Schrijver, C. J., et al. 2016, ApJ, 829, 89

Bk-Stȩślicka, U., Gibson, S. E., Fan, Y., et al. 2013, ApJ, 770, L28

Bemporad, A. 2017, ApJ, 846, 86

Bemporad, A., \& Mancuso, S. 2013, J. Adv. Res., 4, 287

Bemporad, A., Poletto, G., Suess, S. T., et al. 2003, ApJ, 593, 1146
Benevolenskaya, E. E. 2004, A\&A, 428, L5

Benevolenskaya, E. E., Kosovichev, A. G., \& Scherrer, P. H. 2001, ApJ, 554, L107

Berdichevsky, D. B. 2013, Sol. Phys., 284, 245

Billings, D. E. 1966, A Guide to the Solar Corona (New York: Academic Press)

Blanco Rodríguez, J., del Toro Iniesta, J. C., Orozco Suárez, D., et al. 2018, ApJS, 237, 35

Boe, B., Habbal, S., Druckmüller, M., et al. 2018, ApJ, 859, 155

Bommier, V., \& Sahal-Brechot, S. 1982, Sol. Phys., 78, 157

Brosius, J. W., Davila, J. M., Thomas, R. J., \& White, S. M. 1997, ApJ, 488, 488

Brun, A. S., \& Browning, M. K. 2017, Liv. Rev. Sol. Phys., 14, 4

Brun, A. S., Browning, M. K., Dikpati, M., Hotta, H., \& Strugarek, A. 2015, Space Sci. Rev., 196, 101

Burlaga, L., Sittler, E., Mariani, F., \& Schwenn, R. 1981, J. Geophys. Res., 86, 6673

Cameron, R., \& Schüssler, M. 2015, Science, 347, 1333

Canou, A., Amari, T., Bommier, V., et al. 2009, ApJ, 693, L27

Cash, M. D., Biesecker, D. A., Pizzo, V., et al. 2015, Space Weather, 13, 611

Casini, R., \& Judge, P. G. 1999, ApJ, 522, 524

Charbonneau, P. 2010, Liv. Rev. Sol. Phys., 7, 3

Charvin, P. 1965, Ann. Astrophys., 28, 877

Cheung, M. C. M., Boerner, P., Schrijver, C. J., et al. 2015, ApJ, 807, 143

Chifu, I., Inhester, B., \& Wiegelmann, T. 2015, A\&A, 577, A123

Chifu, I., Wiegelmann, T., \& Inhester, B. 2017, ApJ, 837, 10

Colaninno, R. C., Vourlidas, A., \& Wu, C. C. 2013, J. Geophys. Res. (Space Phys.), 118, 6866

Contopoulos, I., Kalapotharakos, C., \& Georgoulis, M. K. 2011, Sol. Phys., 269, 351

Cranmer, S. R., Kohl, J. L., Noci, G., et al. 1999, ApJ, 511, 481

Crosby, N. B., Veronig, A., Robbrecht, E., et al. 2012, in AIP Conf. Ser., eds. Q. Hu, G. Li, G. P. Zank, et al., 1500, 159

Dalmasse, K., Nychka, D., Gibson, S., Fan, Y., \& Flyer, N. 2016, Front. Astron. Space Sci., 3, 24

Dalmasse, K., Savcheva, A., Gibson, S. E., et al. 2019, ApJ, 877, 111

Dasso, S., Nakwacki, M. S., Démoulin, P., \& Mandrini, C. H. 2007, Sol. Phys., 244,115

Davies, J. A., Harrison, R. A., Rouillard, A. P., et al. 2009, Geophys. Res. Lett., 36, L02102

Davies, J. A., Perry, C. H., Trines, R. M. G. M., et al. 2013, ApJ, 777, 167

Davila, J. M. 1994, ApJ, 423, 871

De Pontieu, B., McIntosh, S. W., Carlsson, M., et al. 2007, Science, 318, 1574

De Pontieu, B., Title, A. M., Lemen, J. R., et al. 2014, Sol. Phys., 289, 2733

De Rosa, M. L., Schrijver, C. J., Barnes, G., et al. 2009, ApJ, 696, 1780

DeForest, C. E., Howard, T. A., \& McComas, D. J. 2013, ApJ, 769, 43

Del Zanna, G. 2013, A\&A, 558, A73

Del Zanna, G., \& Mason, H. E. 2018, Liv. Rev. Sol. Phys., 15, 5

Démoulin, P., \& Dasso, S. 2009, A\&A, 507, 969

Dere, K. P., Brueckner, G. E., Howard, R. A., Michels, D. J., \& Delaboudiniere, J. P. 1999, ApJ, 516, 465

Dolei, S., Spadaro, D., \& Ventura, R. 2015, A\&A, 577, A34

Dolei, S., Susino, R., Sasso, C., et al. 2018, A\&A, 612, A84

Dolei, S., Spadaro, D., Ventura, R., et al. 2019, A\&A, 627, A18

Domingo, V., Fleck, B., \& Poland, A. I. 1995, Sol. Phys., 162, 1

Downs, C., Roussev, I. I., van der Holst, B., et al. 2010, ApJ, 712, 1219

Erard, S., Cecconi, B., Le Sidaner, P., et al. 2018, Planet. Space Sci., 150, 65

Farrugia, C. J., Burlaga, L. F., Osherovich, V. A., et al. 1993, J. Geophys. Res., 98,7621

Feng, X., Jiang, C., Xiang, C., Zhao, X., \& Wu, S. T. 2012, ApJ, 758, 62

Fludra, A., \& Landi, E. 2018, in Symposium S335 (Space Weather of the Heliosphere: Processes and Forecasts), Proc. IAU, 13, 87F

Fludra, A., \& Schmelz, J. T. 1999, A\&A, 348, 286

Fox, N. J., Velli, M. C., Bale, S. D., et al. 2016, Space Sci. Rev., 204, 7

Frazin, R. A., \& Kamalabadi, F. 2005a, ApJ, 628, 1061

Frazin, R. A., \& Kamalabadi, F. 2005b, Sol. Phys., 228, 219

Frazin, R. A., Lamy, P., Llebaria, A., \& Vásquez, A. M. 2010, Sol. Phys., 265, 19

Freeland, S. L., \& Handy, B. N. 1998, Sol. Phys., 182, 497

Frutiger, C. 2000, PhD Thesis, ETH Zürich, Switzerland

Génot, V., Jacquey, C., Bouchemit, M., et al. 2010, AdSpR, 45, 1145

Génot, V., Beigbeder, L., Popescu, D., et al. 2018, Planet. Space Sci., 150, 111

Georgoulis, M. K. 2018, in Electric Currents in Geospace and Beyond, eds.

A. Keiling, O. Marghitu, \& M. Wheatland, Geophys. Monograph Ser., 235, 371

Georgoulis, M. K., Bloomfield, D., Piana, M., et al. 2017, AGU Fall Meeting Abstracts

Gibb, G. P. S., Mackay, D. H., Green, L. M., \& Meyer, K. A. 2014, ApJ, 782, 71

Gibson, S. E., Fludra, A., Bagenal, F., et al. 1999, J. Geophys. Res., 104, 9691 
Gibson, S., Kucera, T., White, S., et al. 2016, Front. Astron. Space Sci., 3, 8 Gibson, S. E., Dalmasse, K., Rachmeler, L. A., et al. 2017, ApJ, 840, L13

Gibson, S. E., Vourlidas, A., Hassler, D. M., et al. 2018, Front. Astron. Space Sci., 5, 32

Gilchrist, S. A., Wheatland, M. S., \& Leka, K. D. 2012, Sol. Phys., 276, 133

Gopalswamy, N., Hanaoka, Y., Kosugi, T., et al. 1998, Geophys. Res. Lett., 25, 2485

Gosling, J. T. 1990, Geophys. Monograph Ser., 58, 343

Goucher, G., Love, J., \& Leckner, H. 1994, in Solar-terrestrial Energy program. The initial results from STEP Facilities and Theory Campaigns, 691, proceedings of the 1992 STEP Symposium/5th COSPAR Colloquium (Oxford: Pergamon Press)

Guennou, C., Auchère, F., Soubrié, E., et al. 2012a, ApJS, 203, 25

Guennou, C., Auchère, F., Soubrié, E., et al. 2012b, ApJS, 203, 26

Habbal, S. R., \& Esser, R. 1994, ApJ, 421, L59

Hanle, W. 1924, Z. Phys., 30, 93

Hannah, I. G., \& Kontar, E. P. 2012, A\&A, 539, A146

Hansteen, V. H., \& Leer, E. 1995, J. Geophys. Res., 100, 21577

Hansteen, V., Guerreiro, N., De Pontieu, B., \& Carlsson, M. 2015, ApJ, 811, 106

Harvey, J. W. 1969, PhD Thesis, National Solar Observatory, USA

Harvey, J. W., Hill, F., Hubbard, R. P., et al. 1996, Science, 272, 1284

Hathaway, D. H., \& Upton, L. A. 2016, J. Geophys. Res. (Space Physics), 121, 744

Hayes, A. P., Vourlidas, A., \& Howard, R. A. 2001, ApJ, 548, 1081

Henney, C. J., Hock, R. A., Schooley, A. K., et al. 2015, Space Weather, 13, 141

Hickmann, K. S., Godinez, H. C., Henney, C. J., \& Arge, C. N. 2015, Sol. Phys., 290, 1105

Hidalgo, M. A., Nieves-Chinchilla, T., \& Cid, C. 2002, Geophys. Res. Lett., 29, 1637

Hill, F., Fischer, G., Grier, J., et al. 1994, Sol. Phys., 152, 321

Hill, F., Bogart, R. S., Davey, A., et al. 2004, Proc. SPIE, 5493, 163

Hoeksema, J. T., Wilcox, J. M., \& Scherrer, P. H. 1983, J. Geophys. Res., 88, 9910

Holzer, T. E., \& Leer, E. 1980, J. Geophys. Res., 85, 4665

Horbury, T. S., O’Brien, H., Carrasco Blazquez, I., et al. 2020, A\&A, 642, A9 (Solar Orbiter SI)

Howard, R. A., Moses, J. D., Vourlidas, A., et al. 2008, Space Sci. Rev., 136, 67

Howard, T. A., DeForest, C. E., \& Reinard, A. A. 2012, ApJ, 754, 102

Howard, R. A., Vourlidas, A., Colaninno, R. C., et al. 2020, A\&A, 642, A13 (Solar Orbiter SI)

Huang, X., Wang, H., Xu, L., et al. 2018, ApJ, 856, 7

Hung, C. P., Brun, A. S., Fournier, A., et al. 2017, ApJ, 849, 160

Hyder, C. L., \& Lites, B. W. 1970, Sol. Phys., 14, 147

Inoue, S., Kusano, K., Magara, T., Shiota, D., \& Yamamoto, T. T. 2011, ApJ, 738,161

Isavnin, A. 2016, ApJ, 833, 267

Isavnin, A., Vourlidas, A., \& Kilpua, E. K. J. 2013, Sol. Phys., 284, 203

Isavnin, A., Vourlidas, A., \& Kilpua, E. K. J. 2014, Sol. Phys., 289, 2141

Jacquey, C., Génot, V., Budnik, E., et al. 2010, Astrophys. Space Sci. Proc., 11, 239

Jiang, C., Wu, S. T., Feng, X., \& Hu, Q. 2014, ApJ, 786, L16

Jiang, J., Wang, J.-X., Jiao, Q.-R., \& Cao, J.-B. 2018, ApJ, 863, 159

Judge, P. G. 1998, ApJ, 500, 1009

Judge, P. G., Low, B. C., \& Casini, R. 2006, ApJ, 651, 1229

Kahler, S. W., \& Ling, A. G. 2017, Sol. Phys., 292, 59

Kahler, S. W., \& Webb, D. F. 2007, J. Geophys. Res. (Space Physics), 112, A09103

Kaiser, M. L., Kucera, T. A., Davila, J. M., et al. 2008, Space Sci. Rev., 136, 5

Karak, B. B., \& Miesch, M. 2017, ApJ, 847, 69

Kashyap, V., \& Drake, J. J. 1998, ApJ, 503, 450

Keil, S. L., Rimmele, T. R., Wagner, J., Elmore, D., \& ATST Team 2011, in Solar Polarization 6, eds. J. R. Kuhn, D. M. Harrington, H. Lin, et al., ASP Conf. Ser., 437, 319

Kilpua, E. K. J., Lee, C. O., Luhmann, J. G., \& Li, Y. 2011, Ann. Geophys., 29, 1455

Kontogiannis, I., Georgoulis, M. K., Park, S.-H., \& Guerra, J. A. 2017, Sol Phys., 292, 159

Kontogiannis, I., Georgoulis, M. K., Park, S.-H., \& Guerra, J. A. 2018, Sol Phys, 293, 96

Koomen, M. J., Howard, R. A., \& Michels, D. J. 1998, Sol. Phys., 180, 247

Kouloumvakos, A., Rouillard, A., Wu, Y., et al. 2019, ApJ, 876, 18

Kozarev, K. A., Davey, A., Kendrick, A., Hammer, M., \& Keith, C. 2017, J. Space Weather Space Clim., 7, A32

Kramar, M., Inhester, B., Lin, H., \& Davila, J. 2013, ApJ, 775, 25

Kramar, M., Jones, S., Davila, J., Inhester, B., \& Mierla, M. 2009, Sol. Phys. 259, 109

Kramar, M., Lin, H., \& Tomczyk, S. 2016, ApJ, 819, L36
Krucker, S., Kontar, E. P., Christe, S., \& Lin, R. P. 2007, ApJ, 663, L109

Krucker, S., Hurford, G. J., Grimm, O., et al. 2020, A\&A, 642, A15 (Solar Orbiter SI)

Kuhn, J. R., Penn, M. J., \& Mann, I. 1996, ApJ, 456, L67

Kumar, R., Jouve, L., Pinto, R. F., \& Rouillard, A. P. 2018, Front. Astron. Space Sci., 5,4

Kwon, R.-Y., Zhang, J., \& Olmedo, O. 2014, ApJ, 794, 148

Lamy, P., Damé, L., Vivès, S., \& Zhukov, A. 2010, in Space Telescopes and Instrumentation 2010: Optical, Infrared, and Millimeter Wave, SPIE Conf. Ser., 7731, 773118

Landi, E., Young, P. R., Dere, K. P., Del Zanna, G., \& Mason, H. E. 2013, ApJ, 763,86

Landi, E., Oran, R., Lepri, S. T., et al. 2014, ApJ, 790, 111

Lario, D., Kwon, R.-Y., Riley, P., \& Raouafi, N. E. 2017, ApJ, 847, 103

Laurenza, M., Alberti, T., \& Cliver, E. W. 2018, ApJ, 857, 107

Lee, J., White, S. M., Gopalswamy, N., \& Kundu, M. R. 1997, Sol. Phys., 174, 175

Lee, J., McClymont, A. N., Mikić, Z., White, S. M., \& Kundu, M. R. 1998, ApJ, 501,853

Leer, E., \& Holzer, T. E. 1980, J. Geophys. Res., 85, 4681

Leka, K. D., Barnes, G., Crouch, A. D., et al. 2009, Sol. Phys., 260, 83

Leka, K. D., Barnes, G., \& Wagner, E. 2018, J. Space Weather Space Clim., 8, A25

Liewer, P. C., Hall, J. R., De Jong, M., et al. 2001, J. Geophys. Res., 106, 15903 Liewer, P. C., Hall, J. R., Howard, R. A., et al. 2011, J. Atm. Sol. Terrest. Phys., 73,1173

Lin, R. P., Hurford, G. J., Madden, N. W., et al. 1998, Proc. SPIE, 3442, 2

Lin, H., Penn, M. J., \& Tomczyk, S. 2000, ApJ, 541, L83

Lin, H., Kuhn, J. R., \& Coulter, R. 2004, ApJ, 613, L177

Lindsey, C., \& Braun, D. C. 1997, ApJ, 485, 895

Lionello, R., Linker, J. A., \& Mikić, Z. 2009, ApJ, 690, 902

Liu, C., Deng, N., Wang, J. T. L., \& Wang, H. 2017, ApJ, 843, 104

Löptien, B., Birch, A. C., Gizon, L., et al. 2015, Space Sci. Rev., 196, 251

Low, B. C. 1992, ApJ, 399, 300

Lugaz, N. 2010, Sol. Phys., 267, 411

Lugaz, N., Vourlidas, A., \& Roussev, I. I. 2009, Ann. Geophys., 27, 3479

Lynch, B. J., Li, Y., Thernisien, A. F. R., et al. 2010, J. Geophys. Res. (Space Physics), 115, A07106

Mackay, D. H., \& van Ballegooijen, A. A. 2006, ApJ, 641, 577

Mackay, D. H., Green, L. M., \& van Ballegooijen, A. 2011, ApJ, 729, 97

Mackay, D. H., Yeates, A. R., \& Bocquet, F.-X. 2016, ApJ, 825, 131

MADAWG 2015, Metadata Definition for Solar Orbiter Science Data, Solar Orbiter Technical Note SOL-SGS-TN-0009, ESA/ESAC

Maksimovic, M., Bale, S. D., Chust, T., et al. 2020, A\&A, 642, A12 (Solar Orbiter SI)

Malanushenko, A., Schrijver, C. J., DeRosa, M. L., Wheatland, M. S., \& Gilchrist, S. A. 2012, ApJ, 756, 153

Malanushenko, A., Schrijver, C. J., DeRosa, M. L., \& Wheatland, M. S. 2014, ApJ, 783, 102

Manchester, W., Kilpua, E. K. J., Liu, Y. D., et al. 2017, Space Sci. Rev., 212, 1159

Merkin, V. G., Lyon, J. G., Lario, D., Arge, C. N., \& Henney, C. J. 2016, J. Geophys. Res. (Space Physics), 121, 2866

Metcalf, T. R., Leka, K. D., Barnes, G., et al. 2006, Sol. Phys., 237, 267

Mikić, Z., Linker, J. A., Schnack, D. D., Lionello, R., \& Tarditi, A. 1999, Phys. Plasmas, 6, 2217

Mikić, Z., Downs, C., Linker, J. A., et al. 2018, Nat. Astron., 120

Möstl, C., Farrugia, C. J., Temmer, M., et al. 2009, ApJ, 705, L180

Möstl, C., Amla, K., Hall, J. R., et al. 2014, ApJ, 787, 119

Müller, D., Nicula, B., Felix, S., et al. 2017, A\&A, 606, A13

Müller, D., St. Cyr, O. C., Zouganelis, I., et al. 2020, A\&A, 642, A1 (Solar Orbiter SI)

Nagy, M., Lemerle, A., Labonville, F., Petrovay, K., \& Charbonneau, P. 2017, Sol. Phys., 292, 167

Núñez, M., Klein, K. L., Heber, B., et al. 2018, Solar Particle Radiation Storms Forecasting and Analysis, Astrophysics and Space Science Library (Cham: Springer), 113

Nieves-Chinchilla, T., Colaninno, R., Vourlidas, A., et al. 2012, J. Geophys. Res. (Space Physics), 117, A06106

Nieves-Chinchilla, T., Vourlidas, A., Stenborg, G., et al. 2013, ApJ, 779, 55

Nieves-Chinchilla, T., Linton, M. G., Hidalgo, M. A., et al. 2016, ApJ, 823, 27

Nieves-Chinchilla, T., Vourlidas, A., Raymond, J. C., et al. 2018a, Sol. Phys., 293, 25

Nieves-Chinchilla, T., Linton, M. G., Hidalgo, M. A., \& Vourlidas, A. 2018b, ApJ, 861, 139

Nishizuka, N., Sugiura, K., Kubo, Y., Den, M., \& Ishii, M. 2018, ApJ, 858, 113

Noci, G., Kohl, J. L., \& Withbroe, G. L. 1987, ApJ, 315, 706

Odstrcil, D., \& Pizzo, V. J. 1999, J. Geophys. Res., 104, 28225 
Oran, R., Landi, E., van der Holst, B., et al. 2015, ApJ, 806, 55 Osherovich, V. A., Farrugia, C. J., \& Burlaga, L. F. 1993, AdSpR, 13, 57 Owen, C. J., Bruno, R., Livi, S, et al. 2020, A\&A, 642, A16 (Solar Orbiter SI) Owens, M. J. 2006, J. Geophys. Res. (Space Physics), 111, A12109 Owens, M. J., Lockwood, M., \& Barnard, L. A. 2017, Sci. Rep., 7, 4152 Pagano, P., Mackay, D. H., \& Poedts, S. 2013a, A\&A, 554, A77 Pagano, P., Mackay, D. H., \& Poedts, S. 2013b, A\&A, 560, A38

Pagano, P., Mackay, D. H., \& Poedts, S. 2014, A\&A, 568, A120

Pagano, P., Bemporad, A., \& Mackay, D. H. 2015, A\&A, 582, A72

Pagano, P., Mackay, D. H., \& Yeates, A. R. 2018, J. Space Weather Space Clim., 8, A26

Panasenco, O., Martin, S. F., Velli, M., \& Vourlidas, A. 2013, Sol. Phys., 287, 391

Parker, E. N. 1988, ApJ, 330, 474

Pei, C., Bieber, J. W., Burger, R. A., \& Clem, J. 2012, ApJ, 744, 170

Pence, W. D., Chiappetti, L., Page, C. G., Shaw, R. A., \& Stobie, E. 2010, A\&A 524, A42

Pesnell, W. D., Thompson, B. J., \& Chamberlin, P. C. 2012, Sol. Phys., 275, 3

Petrie, G. J. D. 2015, Liv. Rev. Sol. Phys., 12, 5

Petrovay, K. 2010, Liv. Rev. Sol. Phys., 7, 6

Pinto, R. F., \& Rouillard, A. P. 2017, ApJ, 838, 89

Pinto, R. F., Brun, A. S., Jouve, L., \& Grappin, R. 2011, ApJ, 737, 72

Platten, S. J., Parnell, C. E., Haynes, A. L., Priest, E. R., \& Mackay, D. H. 2014 A\&A, 565, A44

Plotnikov, I., Rouillard, A. P., \& Share, G. H. 2017, A\&A, 608, A43

Plowman, J. 2014, ApJ, 792, 23

Pomoell, J., \& Poedts, S. 2018, J. Space Weather Space Clim, 8, 14

Pottasch, S. R. 1964, MNRAS, 128, 73

Priest, E. R. 1987, Solar Magneto-hydrodynamics (Dordrecht: Kluwer Academic Publishers)

Querfeld, C. W. 1982, ApJ, 255, 764

Rachmeler, L. A., Gibson, S. E., Dove, J. B., DeVore, C. R., \& Fan, Y. 2013, Sol Phys., 288, 617

Rachmeler, L. A., Platten, S. J., Bethge, C., Seaton, D. B., \& Yeates, A. R. 2014, ApJ, 787, L3

Raouafi, N.-E. 2002, A\&A, 386, 721

Raouafi, N. E. 2005, in Chromospheric and Coronal Magnetic Fields, eds. D. E Innes, A. Lagg, \& S. A. Solanki, ESA SP, 596, 3.1

Raouafi, N. E. 2011, in Solar Polarization 6, eds. J. R. Kuhn, D. M. Harrington, H. Lin, et al., ASP Conf. Ser., 437, 99

Raouafi, N.-E., Lemaire, P., \& Sahal-Bréchot, S. 1999, A\&A, 345, 999

Raouafi, N.-E., Sahal-Bréchot, S., \& Lemaire, P. 2002, A\&A, 396, 1019

Raouafi, N. E., Riley, P., Gibson, S., Fineschi, S., \& Solanki, S. K. 2016, Front. Astron. Space Sci., 3, 20

Reinard, A. A., Henthorn, J., Komm, R., \& Hill, F. 2010, ApJ, 710, L121

Rempel, M., \& Cheung, M. C. M. 2014, ApJ, 785, 90

Renotte, E., Buckley, S., Cernica, I., et al. 2016, in Space Telescopes and Instrumentation 2016: Optical, Infrared, and Millimeter Wave, SPIE Conf Ser., 9904, 99043D

Réville, V., \& Brun, A. S. 2017, ApJ, 850, 45

Riethmüller, T. L., Solanki, S. K., Barthol, P., et al. 2017, ApJS, 229, 16

Riley, P., \& Crooker, N. U. 2004, ApJ, 600, 1035

Riley, P., Ben-Nun, M., Linker, J. A., et al. 2014, Sol. Phys., 289, 769

Rochus, P., Auchère, F., Berghmans, D., et al. 2020, A\&A, 642, A8 (Solar Orbiter SI)

Rodkin, D., Goryaev, F., Pagano, P., et al. 2017, Sol. Phys., 292, 90

Rodríguez-Pacheco, J., Wimmer-Schweingruber, R. F., Mason, G. M., et al 2020, A\&A, 642, A7 (Solar Orbiter SI)

Rouillard, A. P. 2011, J. Atm. Sol. Terrest. Phys., 73, 1201

Rouillard, A. P., Davies, J. A., Forsyth, R. J., et al. 2008, Geophys. Res. Lett., 35, L10110

Rouillard, A. P., Davies, J. A., Forsyth, R. J., et al. 2009, J. Geophys. Res. (Space Physics), 114, A07106

Rouillard, A. P., Davies, J. A., Lavraud, B., et al. 2010a, J. Geophys. Res. (Space Physics), 115, A04103

Rouillard, A. P., Lavraud, B., Davies, J. A., et al. 2010b, J. Geophys. Res. (Space Physics), 115, A04104

Rouillard, A. P., Plotnikov, I., Pinto, R. F., et al. 2016, ApJ, 833, 45

Rouillard, A. P., Lavraud, B., Génot, V., et al. 2017, Planet. Space Sci., 147, 61

Ruffenach, A., Lavraud, B., Owens, M. J., et al. 2012, J. Geophys. Res. (Space Physics), 117, A09101

Saez, F., Zhukov, A. N., Lamy, P., \& Llebaria, A. 2005, A\&A, 442, 351

Sahal-Brechot, S., Bommier, V., \& Leroy, J. L. 1977, A\&A, 59, 223

Sanchez-Diaz, E., Rouillard, A. P., Davies, J. A., et al. 2017a, ApJ, 851, 32

Sanchez-Diaz, E., Rouillard, A. P., Davies, J. A., et al. 2017b, ApJ, 835, L7

Sasso, C., Pinto, R. F., Andretta, V., et al. 2019, A\&A, 627, A9

Savani, N. P., Owens, M. J., Rouillard, A. P., Forsyth, R. J., \& Davies, J. A. 2010 ApJ, 714, L128
Scharmer, G. B., Bjelksjo, K., Korhonen, T. K., Lindberg, B., \& Petterson, B. 2003, in Innovative Telescopes and Instrumentation for Solar Astrophysics, eds. S. L. Keil, \& S. V. Avakyan, Proc. SPIE, 4853, 341

Scherrer, P. H., Schou, J., Bush, R. I., et al. 2012, Sol. Phys., 275, 207

Schmelz, J. T., Kashyap, V. L., Saar, S. H., et al. 2009, ApJ, 704, 863

Schmidt, W., von der Lühe, O., Volkmer, R., et al. 2012, Astron. Nachr., 333, 796

Schrijver, C. J., \& De Rosa, M. L. 2003, Sol. Phys., 212, 165

Sheeley, N. R., Walters, J. H., Wang, Y.-M., \& Howard, R. A. 1999, J. Geophys. Res., 104, 24739

Sheeley, Jr., N. R., Herbst, A. D., Palatchi, C. A., et al. 2008, ApJ, 675, 853

Shiota, D., Tsuneta, S., Shimojo, M., et al. 2012, ApJ, 753, 157

Snow, B., Botha, G. J. J., Régnier, S., et al. 2017, ApJ, 842, 16

Solanki, S. K. 1987, Ph.D. Thesis, ETH Zurich

Solanki, S. K., del Toro Iniesta, J. C., Woch, J., et al. 2020, A\&A, 642, A11 (Solar Orbiter SI)

Spadaro, D., Susino, R., Dolei, S., Ventura, R., \& Antonucci, E. 2017, A\&A, 603, A35

SPICE Consortium (Anderson, M., et al.) 2020, A\&A, 642, A14 (Solar Orbiter SI)

Strugarek, A., Beaudoin, P., Charbonneau, P., Brun, A. S., \& do Nascimento, J. D. 2017, Science, 357, 185

Strugarek, A., Beaudoin, P., Charbonneau, P., \& Brun, A. S. 2018, ApJ, 863 , 35

Suess, S. T., Poletto, G., Romoli, M., et al. 2000, J. Geophys. Res., 105, 25033

SunPy Community, Mumford, S. J., Christe, S., et al. 2015, Sci. Discov., 8 , 014009

Tadesse, T., Pevtsov, A. A., Wiegelmann, T., MacNeice, P. J., \& Gosain, S. 2014, Sol. Phys., 289, 4031

Taubman, D. S., \& Marcellin, M. W. 2002, JPEG2000 Image Compression Fundamentals, Standards, and Practice (Dordrecht: Kluwer Academic Publishers)

Thernisien, A., Vourlidas, A., \& Howard, R. A. 2009, Sol. Phys., 256, 111

Thompson, W. T. 2009, Icarus, 200, 351

Thompson, W. T. 2013, Sol. Phys., 283, 489

Titov, V. S., Mikić, Z., Linker, J. A., Lionello, R., \& Antiochos, S. K. 2011, ApJ, 731,111

Titov, V. S., Török, T., Mikic, Z., \& Linker, J. A. 2014, ApJ, 790, 163

Titov, V. S., Downs, C., Mikić, Z., et al. 2018, ApJ, 852, L21

Tomczyk, S., Card, G. L., Darnell, T., et al. 2008, Sol. Phys., 247, 411

Tomczyk, S., Landi, E., Burkepile, J. T., et al. 2016, J. Geophys. Res. (Space Physics), 121, 7470

Tsuneta, S., Ichimoto, K., Katsukawa, Y., et al. 2008a, Sol. Phys., 249, 167

Tsuneta, S., Ichimoto, K., Katsukawa, Y., et al. 2008b, ApJ, 688, 1374

Ulrich, R. K., \& Boyden, J. E. 2006, Sol. Phys., 235, 17

Upton, L., \& Hathaway, D. H. 2014, ApJ, 780, 5

Usmanov, A. V., Matthaeus, W. H., Goldstein, M. L., \& Chhiber, R. 2018, ApJ, 865,25

Valori, G., Kliem, B., \& Keppens, R. 2005, A\&A, 433, 335

van Ballegooijen, A. A. 2004, ApJ, 612, 519

van de Hulst, H. C. 1950, Bull. Astron. Inst. Neth., 11, 135

van der Holst, B., Sokolov, I. V., Meng, X., et al. 2014, ApJ, 782, 81

van Haarlem, M. P., Wise, M. W., Gunst, A. W., et al. 2013, A\&A, 556, A2

Vibert, D., Peillon, C., Lamy, P., Frazin, R. A., \& Wojak, J. 2016, Astron. Comput., 17, 144

Vögler, A., \& Schüssler, M. 2007, A\&A, 465, L43

Vourlidas, A., \& Howard, R. A. 2006, ApJ, 642, 1216

Vourlidas, A., \& Ontiveros, V. 2009, in AIP Conf. Ser., eds. X. Ao, \& G. Z. R. Burrows, 1183, 139

Vourlidas, A., \& Webb, D. F. 2018, ApJ, 861, 103

Vourlidas, A., Colaninno, R., Nieves-Chinchilla, T., \& Stenborg, G. 2011, ApJ, 733, L23

Vourlidas, A., Lynch, B. J., Howard, R. A., \& Li, Y. 2013, Sol. Phys., 284, 179

Vourlidas, A., Howard, R. A., Plunkett, S. P., et al. 2016, Space Sci. Rev., 204, 83

Vásquez, A., Frazin, R., \& Kamalabadi, F. 2009, Sol. Phys., 256, 73

Vásquez, A. M., Huang, Z., Manchester, W. B., \& Frazin, R. A. 2011, Sol. Phys., 274, 259

Walsh, A. P., Horbury, T. S., Maksimovic, M., et al. 2020, A\&A, 642, A5 (Solar Orbiter SI)

Wang, Y.-M., \& Hess, P. 2018, ApJ, 859, 135

Wang, Y.-M., Sheeley, Jr., N. R., Howard, R. A., et al. 1997, ApJ, 485, 875

Wang, Y.-M., Sheeley, Jr., N. R., \& Rich, N. B. 2000, Geophys. Res. Lett., 27, 149

Wang, Y.-M., Sheeley, Jr., N. R., \& Rich, N. B. 2007, ApJ, 658, 1340

Warner, M., Rimmele, T. R., Pillet, V. M., et al. 2018, SPIE Conf. Ser., 10700 $107000 \mathrm{~V}$ 
Warren, H. P., Byers, J. M., \& Crump, N. A. 2017, ApJ, 836, 215

Watson, F. T., Beck, C., Penn, M. J., et al. 2015, Sol. Phys., 290, 3267

Webb, A., Fainberg, J., \& Osherovich, V. 2012, Sol. Phys., 277, 375

Wheatland, M. S., Sturrock, P. A., \& Roumeliotis, G. 2000, ApJ, 540, 1150

White, S. M., \& Kundu, M. R. 1997, Sol. Phys., 174, 31

Wiegelmann, T. 2004, Sol. Phys., 219, 87

Wiegelmann, T., \& Neukirch, T. 2006, A\&A, 457, 1053

Wiegelmann, T., Neukirch, T., Ruan, P., \& Inhester, B. 2007, A\&A, 475, 701

Wiegelmann, T., Yelles Chaouche, L., Solanki, S. K., \& Lagg, A. 2010, A\&A, 511, A4

Withbroe, G. L. 1988, ApJ, 325, 442

Withbroe, G. L., Kohl, J. L., Weiser, H., \& Munro, R. H. 1982, Space Sci. Rev., 33,17

Wood, B. E., Karovska, M., Chen, J., et al. 1999, ApJ, 512, 484

Wood, B. E., Howard, R. A., Thernisien, A., Plunkett, S. P., \& Socker, D. G 2009, Sol. Phys., 259, 163

Wood, B. E., Howard, R. A., Thernisien, A., \& Socker, D. G. 2010, ApJ, 708, L89

Wood, B. E., Wu, C.-C., Lepping, R. P., et al. 2017, ApJS, 229, 29

Worden, J., \& Harvey, J. 2000, Sol. Phys., 195, 247

Yardley, S. L., Mackay, D. H., \& Green, L. M. 2018, ApJ, 852, 82

Yeates, A. R., Mackay, D. H., \& van Ballegooijen, A. A. 2007, Sol. Phys., 245, 87

Yeates, A. R., Mackay, D. H., \& van Ballegooijen, A. A. 2008, Sol. Phys., 247, 103

Yeates, A. R., Attrill, G. D. R., Nandy, D., et al. 2010a, ApJ, 709, 1238

Yeates, A. R., Mackay, D. H., van Ballegooijen, A. A., \& Constable, J. A. 2010b, J. Geophys. Res. (Space Physics), 115, A09112

Yeates, A. R., \& Muñoz-Jaramillo, A. 2013, MNRAS, 436, 3366

Yeates, A. R., Amari, T., Contopoulos, I., et al. 2018, Space Sci. Rev., 214, 99

Zhao, X. P., Hoeksema, J. T., \& Rich, N. B. 2002, AdSpR, 29, 411

Zhu, X., \& Wiegelmann, T. 2018, ApJ, 866, 130

Zhu, X. S., Wang, H. N., Du, Z. L., \& Fan, Y. L. 2013, ApJ, 768, 119

Zlotnik, E. Y. 1968, Sov. Astron., 12, 245

Zouganelis, I., De Groof, A., Walsh, A. P., et al. 2020, A\&A, 642, A3 (Solar Orbiter SI)

1 IRAP, Université de Toulouse, CNRS, CNES, UPS, Toulouse, France

e-mail: alexis . rouillard@irap .omp .eu

2 The Johns Hopkins University Applied Physics Laboratory, Laurel, MD, USA

3 European Space Agency, ESAC, 28692 Villanueva de la Cañada, Madrid, Spain

4 ADNET Systems, Inc., NASA Goddard Spaceflight Center, Code 671, Greenbelt, MD 20771, USA

5 INAF-Turin Astrophysical Observatory, Via Osservatorio 20, 10025 Pino Torinese, TO, Italy

6 INAF-Catania Astrophysical Observatory, Via Santa Sofia 78, 95123 Catania, Italy

7 RAL Space, STFC Rutherford Appleton Laboratory, Harwell, Didcot OX11 0QX, UK

8 Naval Research Laboratory, Space Science Division, Washington, DC 20375, USA

9 University of Applied Sciences and Arts Northwestern Switzerland, 5210 Windisch, Switzerland

10 European Space Agency, ESTEC, PO Box 299, 2200 Noordwijk, The Netherlands

11 Max-Planck-Institut für Sonnensystemforschung, Justus-vonLiebig-Weg 3, 37077 Göttingen, Germany

12 Royal Observatory of Belgium, Ringlaan -3- Av, Circulaire 1180, Brussels, Belgium
13 Southwest Research Institute, 1050 Walnut Street, Boulder, CO, USA

14 Solar Physics Laboratory, Heliophysics Science Division, NASA Goddard Space Flight Center, Greenbelt, MD 2077, USA

15 INAF-Capodimonte Astronomical Observatory, Salita Moiariello 16, 80131 Napoli, Italy

16 Instituto de Astrofísica (IAA-CSIC), Apdo. de Correos 3004, 18080 Granada, Spain

17 UCL-Mullard Space Science Laboratory Holmbury St Mary, Dorking, Surrey RH5 6NT, UK

18 Physikalisch-Meteorologisches Observatorium Davos, World Radiation Center, 7260, Davos Dorf, Switzerland

19 Institut d'Astrophysique Spatiale, CNRS, Univ. Paris-Sud, Université Paris-Saclay, Bât. 121, 91405 Orsay, France

20 Department of Physics and Astronomy - Section of Astronomy and Space Science, University of Firenze, Firenze, Italy

${ }^{21}$ Département d'Astrophysique, Laboratoire AIM Paris-Saclay, CEA/IRFU Université Paris-Diderot CNRS/INSU, 91191 Gif-SurYvette, France

22 Space Vehicles Directorate, Air Force Research Laboratory, Kirtland AFB, New Mexico, USA

23 Department of Physics \& Astronomy, Georgia State University, 30303 Atlanta, GA, USA

24 RCAAM of the Academy of Athens, 11527 Athens, Greece

25 Department of Climate and Space Sciences and Engineering, University of Michigan, Ann Arbor, MI, USA

26 School of Mathematics and Statistics, University of St Andrews, UK

27 Institute of Astronomy and National Astronomical Observatory, Bulgarian Academy of Sciences, Bulgaria

28 Skobeltsyn Institute of Nuclear Physics, Moscow State University, Moscow, Russia

29 Departament de Física Quàntica i Astrofísica, Institut de Ciències del Cosmos (ICCUB), Universitat de Barcelona, Barcelona, Spain

30 Universidad de Alcalá, Space Research Group, 28805 Alcalá de Henares, Spain

31 LESIA, Observatoire de Paris, Université PSL, CNRS, Sorbonne Université, Univ. Paris Diderot, Sorbonne Paris Cité, 5 place Jules Janssen, 92195 Meudon, France

32 Institute for Astronomy, Astrophysics, Space Applications and Remote Sensing (IAASARS), National Observatory of Athens, I. Metaxa \& Vas. Pavlou St., 15236 Penteli, Greece

33 The Catholic University of America, Washington, DC, USA

${ }^{34}$ School of Space Research, Kyung Hee University, Yongin, Gyeonggi-Do 446-701, Republic of Korea

35 Department of Physics, University of Helsinki, Helsinki, Finland

${ }^{36}$ Leibniz-Institut für Astrophysik Potsdam (AIP), Potsdam, Germany

37 Research Software Development Group, Information Service Division, University College London, London, UK

38 School of Physics, Trinity College Dublin, Dublin 2, Ireland

39 School of Cosmic Physics, Dublin Institute of Advanced Studies, Dublin 2, Ireland

40 Predictive Sciences Inc., San Diego, CA, USA

41 Blackett Laboratory, Imperial College London, London, UK

42 Centre de Physique Théorique, Ecole Polytechnique, CNRS, Université Paris-Saclay, Palaiseau, France

43 Laboratoire d'astrophysique de Bordeaux, Univ. Bordeaux, CNRS, B18N, Allée Geoffroy Saint-Hilaire, 33615 Pessac, France 